\title{
Pulse Dynamics in a Three-Component System: Existence Analysis
}

\author{
Arjen Doelman • Peter van Heijster • Tasso J. Kaper
}

Received: 4 August 2007 / Revised: 5 June 2008 / Published online: 5 September 2008

(C) The Author(s) 2008. This article is published with open access at Springerlink.com

\begin{abstract}
In this article, we analyze the three-component reaction-diffusion system originally developed by Schenk et al. (PRL 78:3781-3784, 1997). The system consists of bistable activator-inhibitor equations with an additional inhibitor that diffuses more rapidly than the standard inhibitor (or recovery variable). It has been used by several authors as a prototype three-component system that generates rich pulse dynamics and interactions, and this richness is the main motivation for the analysis we present. We demonstrate the existence of stationary one-pulse and two-pulse solutions, and travelling one-pulse solutions, on the real line, and we determine the parameter regimes in which they exist. Also, for one-pulse solutions, we analyze various bifurcations, including the saddle-node bifurcation in which they are created, as well as the bifurcation from a stationary to a travelling pulse, which we show can be either subcritical or supercritical. For two-pulse solutions, we show that the third component is essential, since the reduced bistable two-component system does not support them. We also analyze the saddle-node bifurcation in which two-pulse solutions are created. The analytical method used to construct all of these pulse solutions is geometric singular perturbation theory, which allows us to show that these solutions lie in the transverse intersections of invariant manifolds in the phase space of the associated six-dimensional travelling wave system. Finally, as we illustrate with numerical simulations, these solutions form the
\end{abstract}

\footnotetext{
A. Doelman · P. van Heijster

Centrum voor Wiskunde en Informatica (CWI), P.O. Box 94079, 1090 GB Amsterdam, The Netherlands

P. van Heijster

e-mail: heijster@cwi.nl
}

\section{A. Doelman}

Korteweg-de Vries Instituut, Universiteit van Amsterdam, Plantage Muidergracht 24, 1018 TV Amsterdam, The Netherlands

e-mail: doelman@cwi.nl

T. J. Kaper $(\varangle)$

Department of Mathematics \& Center for BioDynamics, Boston University, 111 Cummington Street, Boston, MA 02215, USA

e-mail: tasso@math.bu.edu 
backbone of the rich pulse dynamics this system exhibits, including pulse replication, pulse annihilation, breathing pulses, and pulse scattering, among others.

Keywords Three-component reaction-diffusion systems · One-pulse solutions · Travelling pulse solutions · Two-pulse solutions · Geometric singular perturbation theory ·

Melnikov function

AMS (MOS) Subject Classifications Primary: 35K55 - 35B32 - 34C37 - Secondary: $35 \mathrm{~K} 40$

\section{Introduction}

Spatially localized structures, such as fronts, pulses and spots, have been found to exhibit a wide variety of interesting dynamics in dissipative systems. These dynamics include repulsion, annihilation, attraction, breathing, collision, scattering, self-replication, and spontaneous generation. The richness of the observed dynamics typically increases with the complexity and the size of the system. Localized structures, that do not exist in reaction-diffusion (RD) systems with a small number of components, may readily exist when more components and more terms are added to the system. Likewise, solutions that are unstable in small or simple RD systems may become stable with such additions.

The aim of this article is to report on the mathematical analysis of a paradigm example that exhibits this increased richness. In particular, we study the three-component model introduced in [22] and studied further in $[2,15,17,18,24,25]$. In one space dimension, the equations are

$$
\left\{\begin{array}{l}
U_{t}=D_{U} U_{x x}+f(U)-\kappa_{3} V-\kappa_{4} W+\kappa_{1} \\
\tau V_{t}=D_{V} V_{x x}+U-V \\
\theta W_{t}=D_{W} W_{x x}+U-W .
\end{array}\right.
$$

where we used the notation of [15] and we note that (1.1) has the reversibility symmetry $x \rightarrow-x$. Here, the $(U, V)$-subsystem is a classical, bistable two-component RD system, which exhibits dynamics similar to the classical FitzHugh-Nagumo equations (although here $D_{V} \neq 0$, whereas $D_{V}=0$ in FHN), and the variable $W$ denotes an added inhibitor component. We will show that it is responsible for increasing the richness of the types of solutions the model possesses.

In (1.1), $U, V$, and $W$ are real-valued functions of $x \in \mathbb{R}$ and $t \in \mathbb{R}^{+}$, and the subscripts indicate partial derivatives. The parameters $\tau$ and $\theta$ are positive constants, and the primary interest is in using $\tau$ as the bifurcation parameter. The diffusivities of the respective components are denoted by $D_{U}, D_{V}$, and $D_{W}, f(U)$ is a bistable cubic reaction function (often taken to be $\left.f(U)=2 U-U^{3}\right), \kappa_{3}$ and $\kappa_{4}$ denote reaction rates, and $\kappa_{1}$ denotes a constant source term.

The fundamental discovery reported in [22] is that, in this three-component model, the added component $W$ can stabilize stationary and travelling single spot solutions and multispot solutions in two space dimensions, which otherwise are inherently unstable in the classical two-component $(U, V)$-bistable model. This stabilization was shown to occur when $D_{W}$ is sufficiently large relative to $D_{U}$ and $D_{V}$, because then the presence of $W$ prevents spots from extending in the directions perpendicular to their directions of motion. In this manner, $W$ suppresses the instability that spots undergo in two-component systems [22]. 
The dynamics of pulses in the one-dimensional model (1.1) is also known to be richer than in the corresponding one-dimensional version of the two-component model. Pulses collide, scatter, annihilate, among others, as has been shown in $[15,16]$, whereas the dynamics of pulses in the restricted two-component system is much less rich. A special class of unstable two-pulse solutions, called scattors or separators, is identified for $(1.1)$ in $[15,16]$. It is shown that their stable and unstable manifolds organize the evolution in phase space of all nearby solutions. More precisely, during the course of a collision between two pulses, they converge to a separator state, and the location of the initial data relative to the stable and unstable manifolds of this separator determines how and when the pulses scatter off each other. Furthermore, in some parameter regimes, the scattering process may be directed by a combination of two separators, where the colliding pulses first approach one separator, spend a long time near it, and then approach a second separator state, and then finally repel or annihilate, see $[15,16]$.

Our work is inspired by the results from $[18,22]$ and $[15,16]$. We carry out a complementary, rigorous analysis of the existence of certain pulse solutions for a scaled version of the three-component model, see (1.6) below. The model has a rich geometric structure that will be studied using geometric singular perturbation theory, and we note that the application of this theory is challenging due to the fact that the associated ordinary differential equations are six-dimensional.

\subsection{Statement of the Model Equations}

In $[2,15,17,18,22,24,25]$, the numerical values of the diffusivities of the three species differ by several orders of magnitude. For example, in [15], the values are $D_{U}=5 \times 10^{-6}$, $D_{V}=5 \times 10^{-5}$, and $D_{W}=10^{-2}$. Therefore, we are motivated to introduce a scaled spatial variable

$$
\tilde{x}=\frac{x}{\sqrt{D_{V}}}
$$

For computational convenience we also scale out the factor two in the nonlinearity $f(U)=$ $2 U-U^{3}$. Therefore, we introduce

$$
\begin{gathered}
\tilde{t}=2 t, \quad(\tilde{U}, \tilde{V}, \tilde{W})=\frac{1}{2} \sqrt{2}(U, V, W), \quad(\tilde{\tau}, \tilde{\theta})=2(\tau, \theta), \\
\left(\tilde{\kappa}_{1}, \tilde{\kappa}_{3}, \tilde{\kappa}_{4}\right)=\frac{1}{2}\left(\frac{1}{2} \sqrt{2} \kappa_{1}, \kappa_{3}, \kappa_{4}\right) .
\end{gathered}
$$

In terms of these scaled quantities, the system (1.1) is

$$
\left\{\begin{array}{l}
\tilde{U}_{\tilde{t}}=\varepsilon^{2} \tilde{U}_{\tilde{x} \tilde{x}}+\tilde{U}-\tilde{U}^{3}-\tilde{\kappa}_{3} \tilde{V}-\tilde{\kappa}_{4} \tilde{W}+\tilde{\kappa}_{1} \\
\tilde{\tau} \tilde{V}_{\tilde{t}}=\tilde{V}_{\tilde{x} \tilde{x}}+\tilde{U}-\tilde{V} \\
\tilde{\theta} \tilde{W}_{\tilde{t}}=D^{2} \tilde{W}_{\tilde{x} \tilde{x}}+\tilde{U}-\tilde{W}
\end{array}\right.
$$

with the nondimensional diffusivities $\varepsilon^{2}=D_{U} /\left(2 D_{V}\right) \ll 1$ and $D^{2}=D_{W} / D_{V} \gg 1$.

As to the parameters in the reaction terms, the numerical values that are used in [15] are $\left(\kappa_{1}, \kappa_{3}, \kappa_{4}\right)=(-7,1,8.5)$, and very similar values are used in [22]. While these are $\mathcal{O}(1)$ with respect to $\varepsilon$, it is helpful to first study the system with $\mathcal{O}(\varepsilon)$ values of these parameters; i.e., to introduce scaled parameters, as follows:

$$
\tilde{\kappa}_{1}=-\varepsilon \gamma, \quad \tilde{\kappa}_{3}=\varepsilon \alpha, \quad \tilde{\kappa}_{4}=\varepsilon \beta,
$$


where $\alpha, \beta$, and $\gamma$ are $\mathcal{O}(1)$ quantities and where we have taken $\kappa_{1}$ to be negative, since it is negative in all of the above cited articles.

The rationale for this choice of scalings (1.5) is threefold. First, this choice was made to facilitate the mathematical analysis, since in this regime the terms in the $U$-equation corresponding to the source and to the coupling from the inhibitor components are weak, yet not too weak. In fact, the effects of the source and the coupling terms are too weak when they are of $\mathcal{O}\left(\varepsilon^{2}\right)$ [6]. Second, it turns out that much of the rich pulse dynamics exhibited by system (1.4) exists also when the parameters have $\mathcal{O}(\varepsilon)$ values, as we will show in this article (see also [19]). Therefore, one might reasonably hope to understand the origins of the dynamics observed in [15] by beginning with the present analysis. Third, in the numerical simulations of $[22,15]$, which were done on bounded domains, the $W$ variable stays near -0.8 , approximately. Hence, in a very approximate (and rough) sense one might argue, as follows, that there is an effective impact of the parameters in the $U$-equation of (1.4) that is of $\mathcal{O}(\varepsilon)$. Since $\tilde{\kappa}_{3}=0.5$ and $\varepsilon=\frac{1}{10} \sqrt{5} \approx 0.22$, the effect of $V$ in this equation can indeed be considered to be $\mathcal{O}(\varepsilon)$. Moreover, by the scalings (1.3), $\tilde{\kappa}_{4} \tilde{W}-\tilde{\kappa}_{1} \approx 0.07$ for $W=-0.8$ (and $\kappa_{1,4}$ as in [15]), which is clearly also $\mathcal{O}(\varepsilon)$. Thus, it appears that the impact of the source and coupling terms are indeed small.

In light of the above scalings, the model equations that we study are

$$
\left\{\begin{array}{l}
U_{t}=\varepsilon^{2} U_{x x}+U-U^{3}-\varepsilon(\alpha V+\beta W+\gamma) \\
\tau V_{t}=V_{x x}+U-V \\
\theta W_{t}=D^{2} W_{x x}+U-W
\end{array}\right.
$$

where we dropped the tildes. Furthermore, we require that $0<\varepsilon \ll 1,0<\tau, \theta \ll 1 / \varepsilon^{3}$, $D>1$, and $\alpha, \beta, \gamma \in \mathbb{R}$, where the upper bound on $\tau$ and $\theta$ is derived in Sect. 3.1. Moreover, we assume that the solutions $(U(x, t), V(x, t), W(x, t))$ are bounded over the entire domain.

At various stages throughout the analysis, we will see that it is also useful to examine the three-component model in a stretched (or 'fast') spatial variable $\xi=x / \varepsilon$ :

$$
\left\{\begin{array}{l}
U_{t}=U_{\xi \xi}+U-U^{3}-\varepsilon(\alpha V+\beta W+\gamma) \\
\tau V_{t}=\frac{1}{\varepsilon^{2}} V_{\xi \xi}+U-V \\
\theta W_{t}=\frac{D^{2}}{\varepsilon^{2}} W_{\xi \xi}+U-W .
\end{array}\right.
$$

We refer to this system as the fast system, and to system (1.6) as the slow system.

The system (1.6) or (1.7) is well-suited as a paradigm for the analysis of three-component RD systems. On the one hand, it is sufficiently nonlinear and complex so that it supports a rich variety of localized structures, and on the other hand it is sufficiently simple, with linear reaction functions in the second and third components and with linear coupling, so that much of the dynamics can be computed analytically, including certain bifurcations. See also [23]. In this respect, we believe that the results presented here also provide a basis to establish a theory of interacting pulses in this paradigm model.

\subsection{Outline of the Main Results}

We begin in Sect. 2 with examining the stationary, or standing, one-pulse solutions. For these solutions, the $U$-component consists of a front, which connects the (quiescent) state $U=-1+\mathcal{O}(\varepsilon)$ to the (active) state $U=1+\mathcal{O}(\varepsilon)$, and a back, which provides the opposite connection, concatenated together to form a pulse (or homoclinic orbit). Both the front and the back are sharp, so that the pulse is highly localized, due to the asymptotically small value of $\varepsilon^{2}$ in (1.6). The $V$-component of the one-pulse solutions consists of a smooth pulse that 

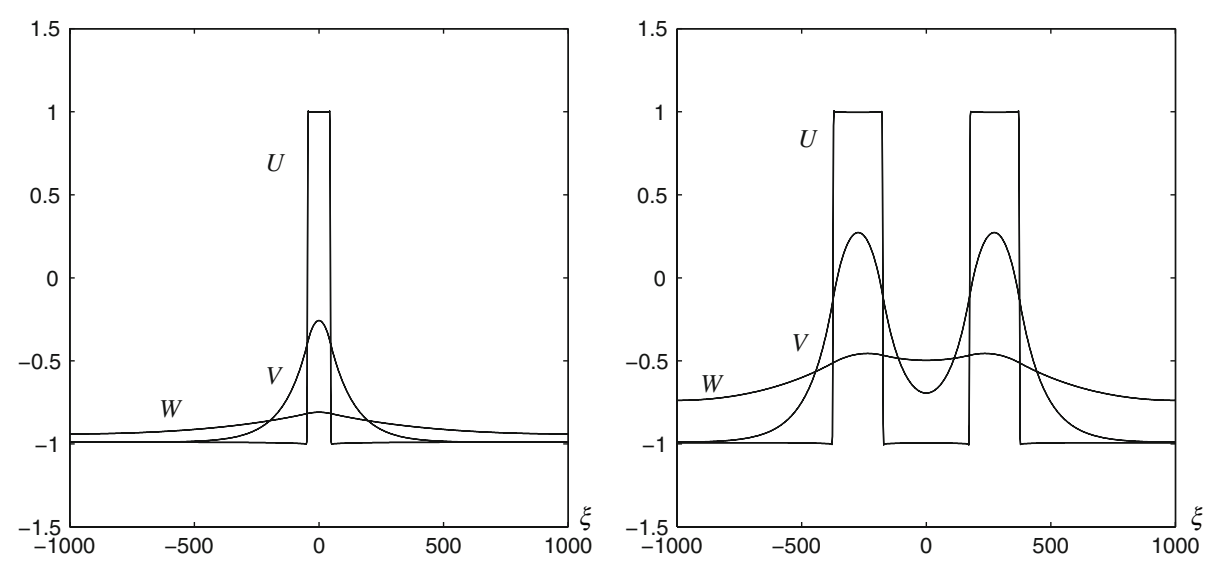

Fig. 1 Stable stationary one-pulse and two-pulse solutions of system (1.6) obtained via numerical simulation. For the one-pulse the system parameters are $(\alpha, \beta, \gamma, D, \tau, \theta, \varepsilon)=(3,1,2,5,1,1,0.01)$, and for the two-pulse we had $(\alpha, \beta, \gamma, D, \tau, \theta, \varepsilon)=(2,-1,-0.25,5,1,1,0.01)$

is centered on the middle of the interval in which the $U$-component is in the active state and that varies over slightly wider interval than the $U$-pulse. Finally, the $W$-component also consists of a single, smooth pulse, but it varies on a wider interval than either of the other two components due to the fact that $D>1$. See Fig. 1. The standing one-pulse solutions are formally constructed in Sect. 2.2. Then, we make this construction rigorous in Theorem 2.1, which states that the three-component model (1.6) possesses standing one-pulse solutions whenever the system parameters satisfy (2.22). See Sect. 2.3 for the statement of this theorem and Sect. 2.4 for its proof.

Next, we analyze the existence of travelling one-pulse solutions. This analysis, presented in Sect. 3, follows the same two-step procedure: we first construct solutions formally (see Sect. 3.1) and then we prove their existence rigorously (see Sects. 3.2 and 3.3). The main result is Theorem 3.1, which states that there exist travelling pulse solutions whenever either $\tau$ or $\theta$ (or both) is $\mathcal{O}\left(1 / \varepsilon^{2}\right)$ and the system parameters satisfy (3.13).

Given these results about standing and travelling one-pulse solutions, it is of interest to investigate the bifurcation of the former into the latter. We do so in Sect. 4. The leading order results are given by (4.2) in Sect. 4.1, and then the rigorous, high-order asymptotics for the main bifurcation parameter $\tau$ as a function of the other parameters is summarized in Lemma 4.1, see Sect. 4.2. It turns out that this bifurcation can be supercritical, as well as subcritical, depending on the parameters, see Corollaries 4.2 and 4.3. This result contrasts with the bifurcation result for the two-dimensional version of this model, obtained in [18], where it was shown that this bifurcation is supercritical.

Having completed our analysis of the one-pulse solutions, we next turn our attention to two-pulse solutions of (1.6). The main result is Theorem 5.1, which guarantees the existence of two-pulse solutions whenever the system parameters satisfy (5.6). These two-pulse solutions have $U$-components that consist of two copies of the $U$-component of the single pulses, while the $V$ - and $W$-components exhibit two peaks as well, but are not near equilibrium in the interval between their two peaks. See Fig. 1. In this sense, the interaction between the pulses is semi-strong, according to the terminology of [3]. We also note that (5.6) is rather complex, and we present investigations of it when $D=2$, and when $D$ is general. Moreover, we give the asymptotics of the key quantities as $D \rightarrow \infty$. See Sects. 5.2 and 5.3, respectively. 
After completing the analysis of these pulse solutions, we examine in Sect. 6 the two-component $(U, V)$-subsystem, obtained from (1.6) by setting $W$ constant at -1 . This analysis of the two-component system enables us to make observations about the differences between the two-component and the three-component systems. For instance, for the two-pulse solutions, we observe that the inclusion of the third component is essential, because the two-component version of the model cannot possess two-pulse solutions. Simply put, there is not enough freedom in the two-component model to permit for the construction of these solutions, and our analysis reveals why the third component—which naturally makes the phase space of the associated ODE problem six-dimensional—creates sufficient space/freedom for their existence.

In Sect.7.1 we present the results of a series of numerical simulations of (1.6). These simulations confirm the various analytical existence and bifurcation results presented herein, and they also reveal the presence of rich pulse interactions, including pulse reflection and annihilation, stable breathing single and double pulses (which bifurcate from stationary pulse solutions), pulse scattering, as well as combinations of these. See Figs. 14-18. The single and double pulses analyzed in this article are key building blocks to understand these rich pulse interactions. Finally, in Sect. 7.2, we summarize our analysis and discuss some related items.

Remark 1.1 The two-pulse solutions constructed in [7,10] for the FHN system differ in several respects from those constructed here. In FHN, these are essentially copies of the onepulse solution, that must be very far apart, and that exhibit oscillatory behavior in the interval between the pulses. The mechanism responsible for their existence is related to the classical Shilnikov mechanism.

Remark 1.2 Other examples of stabilization via the inclusion of an additional component in a model are given for instance by the Gray-Scott and Gierer-Meinhardt systems. In these, one-pulse (homoclinic) solutions that are unstable with respect to the scalar RD equation for the activator component are stabilized in certain parameter regimes by the coupling to the equation for the inhibitory component. The diffusive flux of inhibitor into the pulse domains helps to localize the activator concentration, hence stabilizing one-pulse solutions, and we refer to $[3,5]$ for the mathematical analysis using the Evans function and the stability index. Moreover, it is is worth noting that the converse may also arise; namely in [6] it is shown that stable fronts of a bistable, scalar RD equation are destabilized through coupling to a second component when the parameters are chosen so that either the essential spectrum approaches the origin or an eigenvalue emerges from the essential spectrum and becomes unstable.

\section{Stationary One-Pulse Solutions}

\subsection{Basic Observations}

First, we look at stationary pulses of system (1.7), i.e., we put $\left(U_{t}, V_{t}, W_{t}\right)=(0,0,0)$. By introducing $p=u_{\xi}, q=\frac{1}{\varepsilon} v_{\xi}$ and $r=\frac{D}{\varepsilon} w_{\xi}$, we transform system (1.7) into a six-dimensional singular perturbed ordinary differential equation (ODE)

$$
\left\{\begin{aligned}
u_{\xi} & =p \\
p_{\xi} & =-u+u^{3}+\varepsilon(\alpha v+\beta w+\gamma) \\
v_{\xi} & =\varepsilon q \\
q_{\xi} & =\varepsilon(v-u) \\
w_{\xi} & =\frac{\varepsilon}{D} r \\
r_{\xi} & =\frac{\varepsilon}{D}(w-u) .
\end{aligned}\right.
$$


Although $\xi$ is the spatial variable, it will play the role of 'time' in our analysis. The system possesses two symmetries

$$
\begin{aligned}
& \xi \rightarrow-\xi, p \rightarrow-p, q \rightarrow-q, r \rightarrow-r \\
& u \rightarrow-u, p \rightarrow-p, v \rightarrow-v, q \rightarrow-q, w \rightarrow-w, r \rightarrow-r, \gamma \rightarrow-\gamma .
\end{aligned}
$$

Note that the first symmetry corresponds to the reversibility symmetry $(x, \xi) \rightarrow(-x,-\xi)$ in (1.6) and (1.7), respectively. The fixed points of system (2.1) have $p=q=r=0$, and $u=v=w$ with $u^{3}+u(-1+\varepsilon(\alpha+\beta))+\varepsilon \gamma=0$. Solving this last equation yields

$$
u_{\varepsilon}^{ \pm}= \pm 1 \mp \frac{1}{2} \varepsilon(\alpha+\beta \pm \gamma)+\mathcal{O}\left(\varepsilon^{2}\right), u_{\varepsilon}^{0}=\varepsilon \gamma+\mathcal{O}\left(\varepsilon^{2}\right) \text {. }
$$

Hence, there are three fixed points,

$$
P_{\varepsilon}^{ \pm}=\left(u_{\varepsilon}^{ \pm}, 0, u_{\varepsilon}^{ \pm}, 0, u_{\varepsilon}^{ \pm}, 0\right), P_{\varepsilon}^{0}=\left(u_{\varepsilon}^{0}, 0, u_{\varepsilon}^{0}, 0, u_{\varepsilon}^{0}, 0\right) .
$$

It can be checked [23] that $P_{\varepsilon}^{ \pm}$, respectively $P_{\varepsilon}^{0}$, represent stable, respectively unstable, trivial states of the PDE (1.6) and (1.7).

The fast reduced system (FRS) is obtained by letting $\varepsilon \downarrow 0$ in (2.1),

$$
\left\{\begin{array}{l}
u_{\xi}=p \\
p_{\xi}=-u+u^{3}
\end{array}\right.
$$

as well as $\left(v_{\xi}, q_{\xi}, w_{\xi}, r_{\xi}\right)=(0,0,0,0)$, i.e., $(v, q, w, r) \equiv\left(v_{*}, q_{*}, w_{*}, r_{*}\right)$ with $v_{*}, q_{*}, w_{*}$, $r_{*} \in \mathbb{R}$ constants. The fixed points of the FRS are given by $(u, p) \in\{( \pm 1,0),(0,0)\}$. The former are saddles. The latter, $(0,0)$, is a center that corresponds to $P_{\varepsilon}^{0}$ and thus to an unstable trivial state of (1.6) - we will therefore not consider it.

We define the four-dimensional invariant manifolds $\mathcal{M}_{0}^{ \pm}$by

$$
\mathcal{M}_{0}^{ \pm}:=\left\{(u, p, v, q, r, w) \in \mathbb{R}^{6}: u= \pm 1, p=0\right\},
$$

which are the unions of the saddle points over all possible $v_{*}, q_{*}, w_{*}, r_{*} \in \mathbb{R}$. Planar system (2.5) is integrable with Hamiltonian

$$
H(u, p)=\frac{1}{2}\left(p^{2}+u^{2}\right)-\frac{1}{4}\left(u^{4}+1\right),
$$

which is chosen such that $H(u, p)=0$ on $\mathcal{M}_{0}^{ \pm}$. The FRS possesses heteroclinic orbits $\left(u_{h}^{0, \pm}(\xi), p_{h}^{0, \pm}(\xi)\right)$ that connect the fixed points $(u, p)=( \pm 1,0)$ to $(u, p)=(\mp 1,0)$,

$$
u_{h}^{0, \pm}(\xi)=\mp \tanh \left(\frac{1}{2} \sqrt{2} \xi\right), \quad p_{h}^{0, \pm}(\xi)=\mp \frac{1}{2} \sqrt{2} \operatorname{sech}^{2}\left(\frac{1}{2} \sqrt{2} \xi\right) .
$$

See Fig. 2 . The manifolds $\mathcal{M}_{0}^{ \pm}$are normally hyperbolic, and they have five-dimensional stable and unstable manifolds $W^{u, s}\left(\mathcal{M}_{0}^{ \pm}\right)$that are the unions of the four-parameter $\left(v_{*}, q_{*}, w_{*}, r_{*}\right)$ families of one-dimensional stable and unstable manifolds of the saddle points $(u, p)=$ $( \pm 1,0)$ in $(2.5)$.

Fenichel's first persistence theorem $[8,11,14]$ implies that for $\varepsilon$ small enough, system (2.1) has locally invariant slow manifolds $\mathcal{M}_{\varepsilon}^{ \pm}$which are $\mathcal{O}(\varepsilon) C^{1}$-close to $\mathcal{M}_{0}^{ \pm}$, i.e., $\mathcal{M}_{\varepsilon}^{ \pm}$ can be represented by

$$
\mathcal{M}_{\varepsilon}^{ \pm}:=\left\{u= \pm 1+\varepsilon u_{1}^{ \pm}(v, q, w, r ; \varepsilon), p=\varepsilon p_{1}^{ \pm}(v, q, w, r ; \varepsilon)\right\},
$$




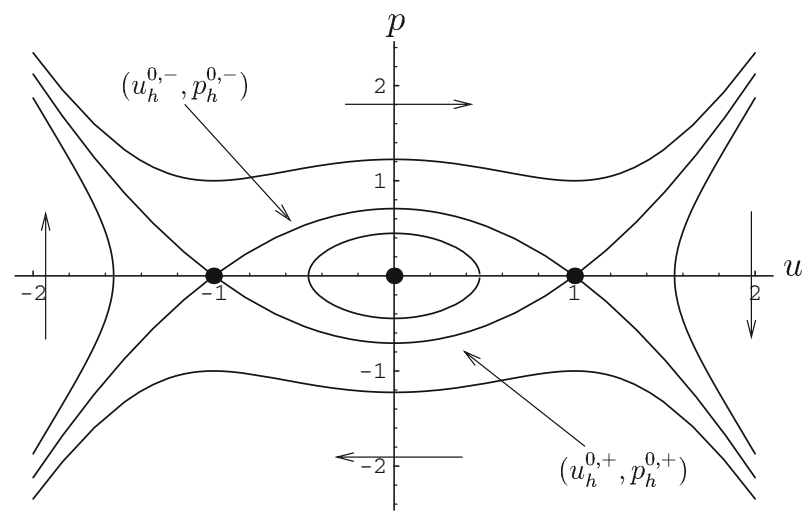

Fig. 2 The phase portrait of the fast reduced Hamiltonian system (2.5)

where the graphs $u_{1}$ and $p_{1}$ can be computed by an expansion in $\varepsilon$,

$$
\mathcal{M}_{\varepsilon}^{ \pm}=\left\{u= \pm 1-\frac{1}{2} \varepsilon(\alpha v+\beta w+\gamma)+\mathcal{O}\left(\varepsilon^{2}\right), p=\mathcal{O}\left(\varepsilon^{2}\right)\right\}
$$

The application of Fenichel's second persistence theorem establishes that $\mathcal{M}_{\varepsilon}^{ \pm}$have fivedimensional stable and unstable manifolds, $W^{s, u}\left(\mathcal{M}_{\varepsilon}^{ \pm}\right)$, that are $\mathcal{O}(\varepsilon) C^{1}$-close to their $\varepsilon=0$ counterparts $W^{u, s}\left(\mathcal{M}_{0}^{ \pm}\right)$. Observe that the critical points $P_{\varepsilon}^{ \pm}$have three-dimensional stable and unstable manifolds $W^{u, s}\left(P_{\varepsilon}^{ \pm}\right)$which are contained in $W^{u, s}\left(\mathcal{M}_{\varepsilon}^{ \pm}\right)$.

There are two slow reduced limit systems (SRS), both of which we write in terms of the fast variable $\xi$ : one that governs the flow on $\mathcal{M}_{\varepsilon}^{-}$,

$$
\left\{\begin{array}{l}
v_{\xi \xi}=\varepsilon^{2}(v+1+\mathcal{O}(\varepsilon)), \\
w_{\xi \xi}=\frac{\varepsilon^{2}}{D^{2}}(w+1+\mathcal{O}(\varepsilon)),
\end{array}\right.
$$

and one that governs the flow on $\mathcal{M}_{\varepsilon}^{+}$,

$$
\left\{\begin{array}{l}
v_{\xi \xi}=\varepsilon^{2}(v-1+\mathcal{O}(\varepsilon)), \\
w_{\xi \xi}=\frac{\varepsilon^{2}}{D^{2}}(w-1+\mathcal{O}(\varepsilon)) .
\end{array}\right.
$$

Observe that $(v, q, w, r)=( \pm 1,0, \pm 1,0)+\mathcal{O}(\varepsilon)$ are saddle points on $\mathcal{M}_{\varepsilon}^{ \pm}$that correspond to the fixed points $P_{\varepsilon}^{ \pm}(2.4)$. Also note that the $v$ - and $w$-equations are decoupled, so that both ODEs can be considered separately. See also Remark 2.1. Hence, we have a $(v, q)$ subsystem and a $(w, r)$-subsystem, both with two saddle points. These four saddle points each have one-dimensional stable and unstable manifolds, $l_{v, w}^{u, s, \pm}$, that are given to leading order by

$$
\begin{aligned}
& \ell_{v}^{u, \pm}=\{q=\mp 1+v\}, \ell_{w}^{u, \pm}=\{r=\mp 1+w\}, \\
& \ell_{v}^{s, \pm}=\{q= \pm 1-v\}, \ell_{w}^{s, \pm}=\{r= \pm 1-w\} .
\end{aligned}
$$

In Fig. 3, we sketch some orbits on the manifolds $\mathcal{M}_{\varepsilon}^{ \pm}$.

\subsection{The Construction of One-Pulse Solutions $\gamma_{h, j}^{-}(\xi)$ Homoclinic to $P_{\varepsilon}^{-}$}

In this section, we consider symmetric standing one-pulse solutions $\gamma_{h, j}^{-}(\xi)$ that are homoclinic to $P_{\varepsilon}^{-}$. Here, we present the formal derivation. Then, in Sect. 2.3, we formulate a 

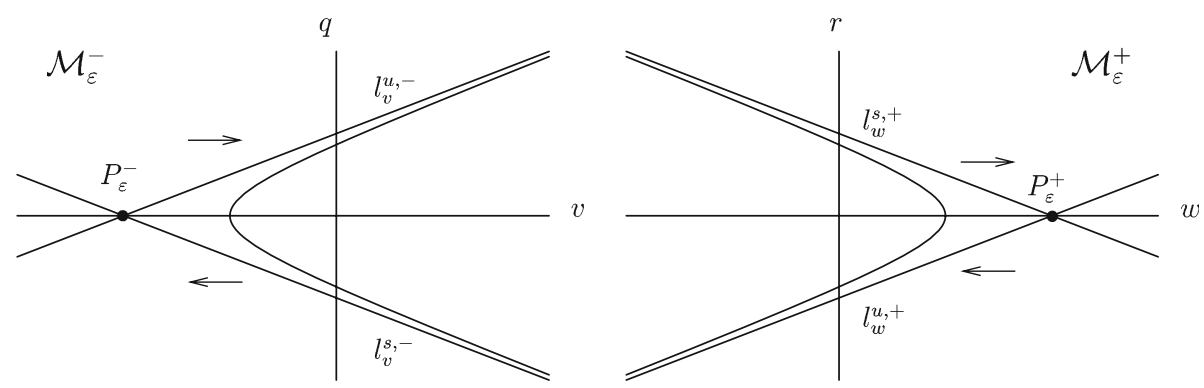

Fig. 3 The flow generated by the $(v, q)$-subsystem on $\mathcal{M}_{\varepsilon}^{-}$and that of the $(w, r)$-subsystem on $\mathcal{M}_{\varepsilon}^{+}$. Note that stable/unstable manifolds $l_{v}^{u, s, \pm}$ and $l_{w}^{u, s, \mp}$ have the same slopes

theorem based on this analysis-Theorem 2.1, and we prove this theorem in Sect. 2.4. This proof also establishes the validity of the asymptotic analysis in this section. Note that orbits homoclinic to the other fixed point $P_{\varepsilon}^{+}$can be obtained from these orbits by application of the symmetries (2.2).

Before we start with the construction of $\gamma_{h, j}^{-}(\xi)$, we introduce some notation. From Figs. 1 to 4 , we notice that there are five different regions, three in which the leading order spatial evolution is given by the SRS (2.10) and (2.11), and two regions that are governed by the FRS (2.5). Since the PDEs are translation invariant, we may parametrize the pulse solution so that its $u, v, w$-components are at a local extremum at $\xi=0$, i.e., $p_{h, j}^{-}(0)=q_{h, j}^{-}(0)=$ $r_{h, j}^{-}(0)=0$ - we will find that $v_{h, j}^{-}(0)$ and $w_{h, j}^{-}(0)$ are maxima, while $u_{h, j}^{-}(0)$ is a (local) minimum. Moreover, we introduce $\xi_{*}$ as the position of the 'jump mid-point(s)', more precisely $\xi_{*}$ is such that $\gamma_{h, j}^{-}(\xi)$ is half-way between the two slow manifolds at $\xi=\xi_{*}$, i.e., $u_{h, j}^{-}=0$ at $\xi= \pm \xi_{*}(2.2)$. We will find that $\xi_{*}=\mathcal{O}\left(\frac{1}{\varepsilon}\right)$, but at this point of the analysis it is still undetermined. Next, we define the two 'fast intervals' $I_{f}^{\mp}$ and the three 'slow intervals' $I_{s}^{\mp}, I_{s}^{0}$,

$$
\begin{aligned}
I_{f}^{-} & :=\left(-\xi_{*}-\frac{1}{\sqrt{\varepsilon}},-\xi_{*}+\frac{1}{\sqrt{\varepsilon}}\right), I_{f}^{+}:=\left(\xi_{*}-\frac{1}{\sqrt{\varepsilon}}, \xi_{*}+\frac{1}{\sqrt{\varepsilon}}\right), \\
I_{s}^{-} & :=\left(-\infty,-\xi_{*}-\frac{1}{\sqrt{\varepsilon}}\right], I_{s}^{0}:=\left[-\xi_{*}+\frac{1}{\sqrt{\varepsilon}}, \xi_{*}-\frac{1}{\sqrt{\varepsilon}}\right], I_{s}^{+}:=\left[\xi_{*}+\frac{1}{\sqrt{\varepsilon}}, \infty\right) .
\end{aligned}
$$

Note that the choice of the width for $I_{f}^{ \pm}$of $2 / \sqrt{\varepsilon}$ is standard, but arbitrary. We can now give a more precise definition of the five regions mentioned above (see Fig. 4).

1. The dynamics take place exponentially close to the slow manifold $\mathcal{M}_{\varepsilon}^{-}: \xi \in I_{s}^{-}$.

2. The dynamics take place in the fast field: $\xi \in I_{f}^{-}$.

3. The dynamics take place exponentially close to $\mathcal{M}_{\varepsilon}^{+}: \xi \in I_{s}^{0}$.

4. The dynamics take place in the fast field: $\xi \in I_{f}^{+}$.

5. The dynamics take place exponentially close to $\mathcal{M}_{\varepsilon}^{-}: \xi \in I_{s}^{+}$.

By definition,

$\gamma_{h, j}^{-}=\left(u_{h, j}^{-}, p_{h, j}^{-}, v_{h, j}^{-}, q_{h, j}^{-}, w_{h, j}^{-}, r_{h, j}^{-}\right) \in W^{u}\left(P_{\varepsilon}^{-}\right) \cap W^{s}\left(P_{\varepsilon}^{-}\right) \subset W^{u}\left(\mathcal{M}_{\varepsilon}^{-}\right) \cap W^{s}\left(\mathcal{M}_{\varepsilon}^{-}\right)$,

while the jump mid-points are defined by

$$
\gamma_{h, j}^{-}\left( \pm \xi_{*}\right)=\left(0, \mp p_{*}, v_{*}, \mp q_{*}, w_{*}, \mp r_{*}\right) .
$$




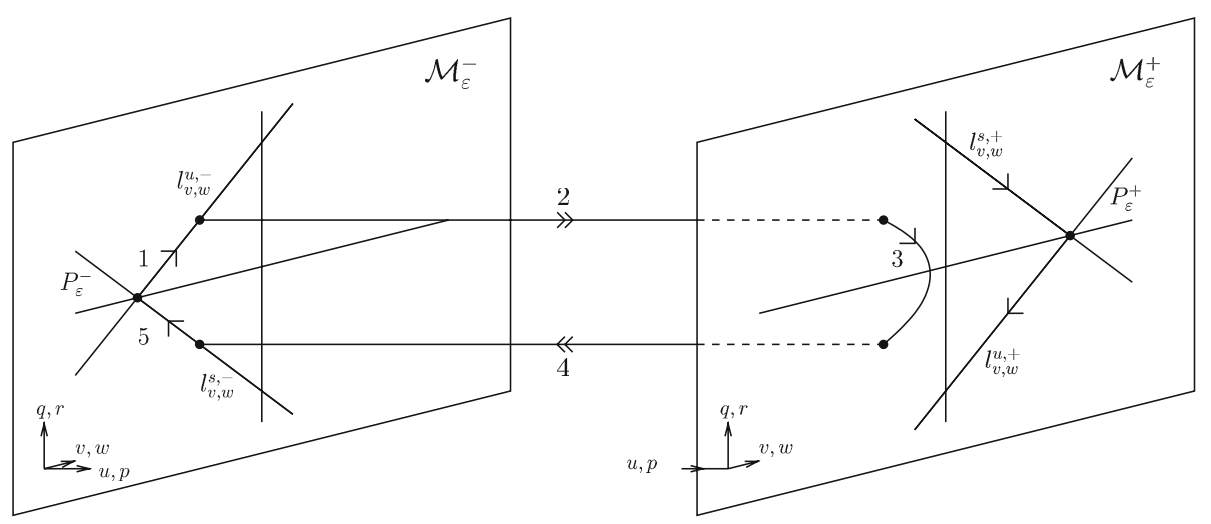

Fig. 4 A schematic sketch of a standing pulse solution $\gamma_{h, j}^{-}(\xi)$ in the six-dimensional $(u, p, v, q, w, r)-$ phase space. In region 1 , the pulse is exponentially close to $\mathcal{M}_{\varepsilon}^{-}$for a long 'spatial time' and approaches $P_{\varepsilon}^{-}$ as $\xi \rightarrow-\infty$. It 'takes off' from $\mathcal{M}_{\varepsilon}^{-}$at $\xi=-\xi_{*}-\frac{1}{\sqrt{\varepsilon}}$ (by definition) and 'jumps' through the fast field $\left(\xi \in I_{f}^{-}\right.$) towards $\mathcal{M}_{\varepsilon}^{+}$-this is region 2. In region $3, \gamma_{h, j}^{-}(\xi)$ touches down near $\mathcal{M}_{\varepsilon}^{+}$at $\xi=-\xi_{*}+\frac{1}{\sqrt{\varepsilon}}$ and remains exponentially close to $\mathcal{M}_{\varepsilon}^{+}$until $\xi=\xi_{*}-\frac{1}{\sqrt{\varepsilon}}$, from where it jumps back towards $\mathcal{M}_{\varepsilon}^{-}$, which defines region $4\left(\xi \in I_{f}^{+}\right.$). In the final region, $5, \gamma_{h, j}^{-}(\xi)$ is again exponentially close to $\mathcal{M}_{\varepsilon}^{-}$and approaches $P_{\varepsilon}^{-}$as $\xi \rightarrow \infty$. See also Fig. 1 in which $\gamma_{h, j}^{-}(\xi)$ exhibits the same structure

Furthermore, since $\gamma_{h, j}^{-}(\xi)$ remains exponentially close to $\mathcal{M}_{\varepsilon}^{+}$for $\xi \in I_{s}^{0}, \gamma_{h, j}(\xi)$ is also exponentially close to $W^{u}\left(P_{\varepsilon}^{-}\right) \cap W^{s}\left(\mathcal{M}_{\varepsilon}^{+}\right)$and to $W^{s}\left(P_{\varepsilon}^{-}\right) \cap W^{u}\left(\mathcal{M}_{\varepsilon}^{+}\right)$for sufficiently long time. Note that $\gamma_{h, j}^{-}(\xi) \notin W^{u}\left(\mathcal{M}_{\varepsilon}^{-}\right) \cap W^{s}\left(\mathcal{M}_{\varepsilon}^{+}\right)$or $W^{s}\left(\mathcal{M}_{\varepsilon}^{-}\right) \cap W^{u}\left(\mathcal{M}_{\varepsilon}^{+}\right)$, since it has to be able to jump back again from $\mathcal{M}_{\varepsilon}^{+}$to $\mathcal{M}_{\varepsilon}^{-}$.

By considering possible take off and touch down points of jumps through the fast field and by studying, in fact explicitly solving, the slow flows on $\mathcal{M}_{\varepsilon}^{-}(2.10)$ and on $\mathcal{M}_{\varepsilon}^{+}$(2.11), we obtain relations between the coordinates $\left(v_{*}, \mp q_{*}, w_{*}, \mp r_{*}\right)$ of the jump mid-points and their spatial positions $\pm \xi_{*}$ that uniquely determine the homoclinic orbit(s) $\gamma_{h, j}^{-}(\xi)$; see Remark 2.1 .

For $\varepsilon \neq 0$, the Hamiltonian $H(u, p)(2.6)$ is not conserved

$$
\begin{aligned}
\frac{d}{d \xi} H(u(\xi), p(\xi)) & =u u_{\xi}+p p_{\xi}-u^{3} u_{\xi} \\
& =u p+p\left(-u+u^{3}+\varepsilon(\alpha v+\beta w+\gamma)\right)-u^{3} p \\
& =\varepsilon p(\alpha v+\beta w+\gamma) .
\end{aligned}
$$

Since $\left(u_{h, j}^{-}(\xi), p_{h, j}^{-}(\xi)\right)$ must be $\mathcal{O}(\varepsilon)$ close to the heteroclinic solution $\left(u_{h}^{0,-}(\xi), p_{h}^{0,-}(\xi)\right)$ (2.7) of the FRS (2.5) in the fast field $I_{f}^{-}$, the total change in $H$ for an orbit $\gamma_{h, j}^{-}(\xi)$ that jumps from $\mathcal{M}_{\varepsilon}^{-}$to $\mathcal{M}_{\varepsilon}^{+}$is approximated by

$$
\begin{aligned}
\Delta_{f}^{-} H\left(v_{*}, q_{*}, w_{*}, r_{*}\right) & =\int_{I_{f}^{-}} H_{\xi} d \xi \\
& =\int_{I_{f}^{-}} \varepsilon p_{h}^{0,-}\left(\xi+\xi_{*}\right)\left(\alpha v_{*}+\beta w_{*}+\gamma\right) d \xi+\mathcal{O}(\varepsilon \sqrt{\varepsilon})
\end{aligned}
$$




$$
\begin{aligned}
& =\varepsilon\left(\alpha v_{*}+\beta w_{*}+\gamma\right) \int_{-\infty}^{\infty} p_{h}^{0,-}(\xi) d \xi+\mathcal{O}(\varepsilon \sqrt{\varepsilon}) \\
& =2 \varepsilon\left(\alpha v_{*}+\beta w_{*}+\gamma\right)+\mathcal{O}(\varepsilon \sqrt{\varepsilon}),
\end{aligned}
$$

where we have used (2.7), (2.14), and assumed that $\xi_{*}=\mathcal{O}\left(\frac{1}{\varepsilon}\right)$. Note that $\Delta_{f}^{-} H$ in principle depends on $\left(v_{*}, q_{*}, w_{*}, r_{*}\right)$, the slow $(v, q, w, r)$-coordinates of the jump mid-points, and that these coordinates do not vary to leading order during a jump through the fast field,

$$
\begin{aligned}
& \Delta_{f}^{-} v=\int_{I_{f}^{-}} v_{\xi} d \xi=\int_{I_{f}^{-}} \varepsilon q d \xi=2 q_{*} \sqrt{\varepsilon}+\mathcal{O}(\varepsilon)=\mathcal{O}(\sqrt{\varepsilon}), \\
& \Delta_{f}^{-} q=\int_{I_{f}^{-}} q_{\xi} d \xi=\int_{I_{f}^{-}} \varepsilon(v-u) d \xi=2 v_{*} \sqrt{\varepsilon}+\mathcal{O}(\varepsilon)=\mathcal{O}(\sqrt{\varepsilon}), \\
& \Delta_{f}^{-} w=\int_{I_{f}^{-}} w_{\xi} d \xi=\int_{I_{f}^{-}} \frac{\varepsilon}{D} r d \xi=2 r_{*} \frac{1}{D} \sqrt{\varepsilon}+\mathcal{O}(\varepsilon)=\mathcal{O}(\sqrt{\varepsilon}), \\
& \Delta_{f}^{-} r=\int_{I_{f}^{-}} r_{\xi} d \xi=\int_{I_{f}^{-}} \frac{\varepsilon}{D}(w-u) d \xi=2 w_{*} \frac{1}{D} \sqrt{\varepsilon}+\mathcal{O}(\varepsilon)=\mathcal{O}(\sqrt{\varepsilon}) .
\end{aligned}
$$

On the other hand, such an orbit $\gamma_{h, j}^{-}(\xi)$ cannot have a total change of more than $\mathcal{O}\left(\varepsilon^{2}\right)$ over a jump through the fast field $I_{f}^{-}$, since

$$
\begin{aligned}
\left.H(u, p)\right|_{\mathcal{M}_{\varepsilon}^{ \pm}}= & \frac{1}{2}\left(\left( \pm 1-\frac{1}{2} \varepsilon(\alpha v+\beta w+\gamma)+\mathcal{O}\left(\varepsilon^{2}\right)\right)^{2}+\mathcal{O}\left(\varepsilon^{2}\right)^{2}\right) \\
& -\frac{1}{4}\left(\left( \pm 1-\frac{1}{2} \varepsilon(\alpha v+\beta w+\gamma)+\mathcal{O}\left(\varepsilon^{2}\right)\right)^{4}+1\right) \\
= & \frac{1}{2} \mp \frac{1}{2} \varepsilon(\alpha v+\beta w+\gamma)-\frac{1}{4} \pm \frac{1}{2} \varepsilon(\alpha v+\beta w+\gamma)-\frac{1}{4}+\mathcal{O}\left(\varepsilon^{2}\right)=\mathcal{O}\left(\varepsilon^{2}\right),
\end{aligned}
$$

where we recall (2.8) and (2.9). Thus, we conclude that for an orbit $\gamma_{h, j}^{-}(\xi)$ that jumps from $\mathcal{M}_{\varepsilon}^{-}$to $\mathcal{M}_{\varepsilon}^{+}$the following relation for the slow $\left(v_{*}, q_{*}, w_{*}, r_{*}\right)$-coordinates of the jump mid-point must hold to leading order

$$
\alpha v_{*}+\beta w_{*}+\gamma=0
$$

Note that $\Delta_{f}^{-} H\left(v_{*}, q_{*}, w_{*}, r_{*}\right)$ is in fact a Melnikov function that measures the distance between $W^{u}\left(\mathcal{M}_{\varepsilon}^{-}\right)$and $W^{s}\left(\mathcal{M}_{\varepsilon}^{+}\right)$as they intersect the $\{u=0\}$ hyperplane (see [3,6,20]). Condition (2.17) determines the three-dimensional set of initial conditions in $\{u=0\}$ that defines the four-dimensional intersection of the two five-dimensional manifolds $W^{u}\left(\mathcal{M}_{\varepsilon}^{-}\right)$ and $W^{s}\left(\mathcal{M}_{\varepsilon}^{+}\right)$(recall that the phase space is six-dimensional and that the $p$-coordinates of these initial conditions are necessarily $\mathcal{O}(\varepsilon)$ close to $\left.p_{h}^{0,-}(0)=\frac{1}{2} \sqrt{2}(2.7)\right)$.

By the reversibility symmetry (2.2), we know that (2.17) also must hold for the $\left(v_{*},-q_{*}\right.$, $\left.w_{*},-r_{*}\right)$-coordinates, which are the coordinates of the jump mid-points of the orbits that jump from $\mathcal{M}_{\varepsilon}^{+}$to $\mathcal{M}_{\varepsilon}^{-}$near $\xi=\xi_{*}$.

Next, we study the slow flows on $\mathcal{M}_{\varepsilon}^{ \pm}$. The Eqs. 2.10 and 2.11 for these flows are linear and decoupled, thus we may solve for $v$ and $w$ separately. Based on the above analysis, we write down the following boundary conditions for the solutions in regions 1, 3, and 5: 


$$
\begin{aligned}
& v_{h}( \pm \infty)=-1, \quad v_{h}\left(-\xi_{*} \pm \frac{1}{\sqrt{\varepsilon}}\right)=v_{h}\left(\xi_{*} \mp \frac{1}{\sqrt{\varepsilon}}\right)=v_{*}+\mathcal{O}(\sqrt{\varepsilon}), \\
& q_{h}( \pm \infty)=0, \quad q_{h}\left(-\xi_{*} \pm \frac{1}{\sqrt{\varepsilon}}\right)=-q_{h}\left(\xi_{*} \mp \frac{1}{\sqrt{\varepsilon}}\right)=q_{*}+\mathcal{O}(\sqrt{\varepsilon}), \\
& w_{h}( \pm \infty)=-1, w_{h}\left(-\xi_{*} \pm \frac{1}{\sqrt{\varepsilon}}\right)=w_{h}\left(\xi_{*} \mp \frac{1}{\sqrt{\varepsilon}}\right)=w_{*}+\mathcal{O}(\sqrt{\varepsilon}), \\
& r_{h}( \pm \infty)=0, \quad r_{h}\left(-\xi_{*} \pm \frac{1}{\sqrt{\varepsilon}}\right)=-r_{h}\left(\xi_{*} \mp \frac{1}{\sqrt{\varepsilon}}\right)=r_{*}+\mathcal{O}(\sqrt{\varepsilon}),
\end{aligned}
$$

see Figs. 1 and 4. Note that there are more (boundary) conditions than free parameters in the general solutions of (2.10) and (2.11). As a consequence, we find that both $v_{*}$ and $q_{*}$, as well as $w_{*}$ and $r_{*}$, must be related,

$$
q_{*}=v_{*}+1, r_{*}=w_{*}+1,
$$

which in geometrical terms is equivalent to $\left(v_{*}, q_{*}\right) \in \ell_{v}^{u,-}$, and $\left(w_{*}, r_{*}\right) \in \ell_{w}^{u,-}(2.12)$, see also Fig. 3. Moreover, (2.18) yields additional relations between $v_{*}$ and $\xi_{*}$ and between $w_{*}$ and $\xi_{*}$,

$$
v_{*}=-A^{2}, w_{*}=-A^{\frac{2}{D}} \text { where } A=e^{-\varepsilon \xi_{*}} .
$$

Observe that, since $\xi_{*}>0, A \in(0,1)$, so that $v_{*}, w_{*} \in(-1,0)$. For $\left(v_{*}, q_{*}, w_{*}, r_{*}\right)$ and $\xi_{*}$ that satisfy (2.18), (2.19) and (2.20), we obtain the explicit (slow) solutions,

$$
v_{h}(\xi)=\left\{\begin{array}{r}
2 e^{\varepsilon \xi} \sinh \varepsilon \xi_{*}-1 \text { in } 1, \\
-2 e^{-\varepsilon \xi_{*}} \cosh \varepsilon \xi+1 \text { in } 3, \\
2 e^{-\varepsilon \xi} \sinh \varepsilon \xi_{*}-1 \text { in } 5,
\end{array} \quad w_{h}(\xi)=\left\{\begin{array}{r}
2 e^{\frac{\varepsilon}{D} \xi} \sinh \frac{\varepsilon}{D} \xi_{*}-1 \text { in } 1, \\
-2 e^{-\frac{\varepsilon}{D} \xi_{*}} \cosh \frac{\varepsilon}{D} \xi+1 \text { in } 3, \\
2 e^{-\frac{\varepsilon}{D} \xi} \sinh \frac{\varepsilon}{D} \xi_{*}-1 \text { in } 5
\end{array}\right.\right.
$$

to leading order in $\varepsilon$. Thus, together with the Melnikov condition (2.17), the boundary conditions (2.18) imply three relations between $v_{*}, w_{*}$, and $\xi_{*}$. These relations combine into the following jump condition on $A$,

$$
\alpha A^{2}+\beta A^{\frac{2}{D}}=\gamma+\mathcal{O}(\sqrt{\varepsilon}) .
$$

A solution $A \in(0,1)$ of this equation uniquely determines the jump mid-points ( $v_{*}, \mp q_{*}, w_{*}$, $\left.\mp r_{*}\right)$ in phase space of a homoclinic solution $\gamma_{h, j}^{-}(\xi)$, as well as their spatial positions $\pm \xi_{*}$ (2.20).

Remark 2.1 We comment briefly on the coupling between the $V$ - and $W$-components and on the related fact that the homoclinic orbits are isolated. In the PDE (1.7), the variables $V$ and $W$ seem to be only coupled through the equation for $U$. In the construction of $\gamma_{h, j}^{-}(\xi)$, this coupling induces the Melnikov condition (2.17) and gives a natural relationship between

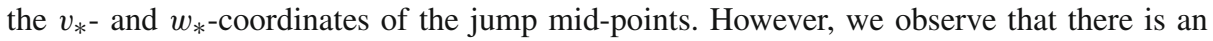
additional geometrically induced coupling between these two components that is not directly obvious from the equations. In particular, the jump mid-points $\xi_{*}$ must be the same for both the $v$-and $w$-components in (2.1), which implies that also the 'time-of-flight' along the slow manifolds must be the same for both the $v$-and $w$-components, since the parametrizations of all of the components of a homoclinic orbit $\gamma_{h, j}^{-}(\xi)$ are of course the same. Hence, from among the entire one-parameter set of pairs $\left(v_{*}, w_{*}\right)$ that satisfy the Melnikov condition (2.17), a unique pair, with $v_{*}=-\left(-w_{*}\right)^{D}(2.20)$, is selected by this 'time-of-flight' constraint. Together, the two constraints determine the values of $v_{*}$ and $w_{*}$ uniquely and thus establish that the homoclinic orbits are isolated. 


\subsection{Existence Theorem}

Based on the analysis of the previous section, we can formulate the following existence result:

Theorem 2.1 Let $(\alpha, \beta, \gamma, D, \tau, \theta, \varepsilon)$ be such that (2.22) has $K$ solutions $A_{j} \in(0,1)(K \in$ $\{0,1,2\})$, and let $\varepsilon$ be small enough. If $K=0$, there are no symmetric orbits homoclinic to $P_{\varepsilon}^{-}$in system (2.1). If $K>0$, then there are $K$ symmetric homoclinic orbits $\gamma_{h, j}^{-}(\xi), j \in$ $\{1, K\}$ to $P_{\varepsilon}^{-}$that have a structure as sketched in Fig. 4, i.e., the orbits $\gamma_{h, j}^{-}(\xi)$ consist of five distinct parts, two fast parts in which it is $\mathcal{O}(\varepsilon)$ close to a fast reduced heteroclinic orbits $\left(u_{h}^{0, \mp}\left(\xi \mp \xi_{*}\right), p_{h}^{0, \pm}\left(\xi \mp \xi_{*}\right), v_{*}, \pm q_{*}, w_{*}, \pm r_{*}\right)(2.7)$ with $\left(v_{*}, q_{*}, w_{*}, r_{*}\right)$ given by (2.19) and (2.20), and three slow parts in which $\left(u_{h, j}^{-}(\xi), p_{h, j}^{-}(\xi)\right)=( \pm 1,0)+\mathcal{O}(\varepsilon)$ and $\left(v_{h, j}^{-}(\xi), q_{h, j}^{-}(\xi), w_{h, j}^{-}(\xi), r_{h, j}^{-}(\xi)\right)$ are given by $(2.21)$, up to $\mathcal{O}(\sqrt{\varepsilon})$-corrections, with

$$
\xi_{*}=\xi_{*, j}=-\frac{1}{\varepsilon} \log A_{j}=\mathcal{O}\left(\frac{1}{\varepsilon}\right) .
$$

The orbits $\gamma_{h, j}^{-}(\xi)$ correspond to stationary pulse solutions

$$
(U(\xi, t), V(\xi, t), W(\xi, t)) \equiv\left(u_{h, j}(\xi), v_{h, j}(\xi), w_{h, j}(\xi)\right),
$$

of (1.7).

Moreover, if $|\alpha D|>|\beta|$ and $\operatorname{sgn}(\alpha) \neq \operatorname{sgn}(\beta)$, then a saddle-node bifurcation of homoclinic orbits occurs, to leading order in $\varepsilon$, as $\gamma$ crosses through

$$
\begin{aligned}
& \gamma_{c 1}(\alpha, \beta, D)=(-\alpha)^{-\frac{1}{D-1}} \beta^{\frac{D}{D-1}}\left(D^{-\frac{1}{D-1}}-D^{-\frac{D}{D-1}}\right)>0 \text { for } \alpha<0<\beta, \\
& \gamma_{c 2}(\alpha, \beta, D)=\alpha^{-\frac{1}{D-1}}(-\beta)^{\frac{D}{D-1}}\left(D^{-\frac{D}{D-1}}-D^{-\frac{1}{D-1}}\right)<0 \text { for } \beta<0<\alpha .
\end{aligned}
$$

The explicit expressions for the values $\gamma_{c 1,2}$ of the saddle-node bifurcations are based on a straightforward leading order analysis: set the partial derivative of (2.22) with respect to $A$ equal to zero to obtain

$$
A_{c}=A_{1}(\alpha, \beta, D)=\left(-\frac{\alpha D}{\beta}\right)^{-\frac{1}{2} \frac{D}{D-1}} \in(0,1),
$$

and then insert this expression back into formula (2.22) to obtain $\gamma_{c 1,2}(2.24)$.

In Fig. 5, the relations between $A_{j}$ and $\gamma$ as solutions of (2.22) have been plotted. The two saddle-node cases at $A_{c}$ described by the theorem are also clearly visible. Two other
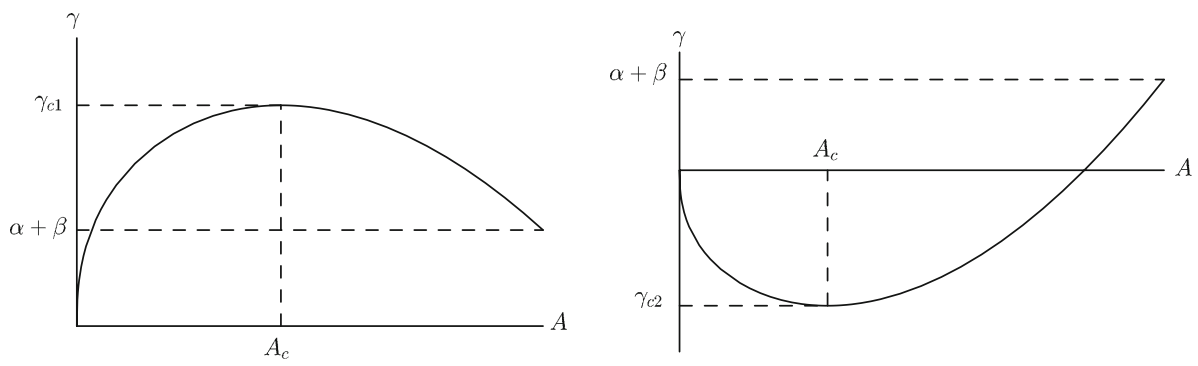

Fig. 5 A graphical representation of the jump condition (2.22) and the associated saddle-node bifurcations as described by Theorem 2.1 for $\alpha<0<\beta$ (with $\alpha+\beta>0$ ) and for $\beta<0<\alpha$ (also with $\alpha+\beta>0$ ). Note that $A_{K} \in(0,1)$ for all parameter combinations 
bifurcations occur: one at $\gamma=A=0$, which corresponds to $\xi_{*}=\infty$ (2.23), i.e., the plateau at which the $U$-component of the one-pulse solution is near 1 becomes infinitely long; the other at $\gamma=\alpha+\beta, A=1$, where the pulse becomes infinitely thin - see also Lemma 2.1 below.

\subsection{The Proof of Theorem 2.1}

The existence of the homoclinic orbit $\gamma_{h, j}^{-}(\xi) \subset W^{u}\left(P_{\varepsilon}^{-}\right) \cap W^{s}\left(P_{\varepsilon}^{-}\right)$will be established by studying $W^{u}\left(\mathcal{M}_{\varepsilon}^{-}\right)$and $W^{u}\left(P_{\varepsilon}^{-}\right)$as they pass along $\mathcal{M}_{\varepsilon}^{+}$. The reversibility symmetry (2.2) plays a crucial role in the proof.

The manifold $W^{u}\left(P_{\varepsilon}^{-}\right)$is three-dimensional, so that all orbits $\gamma_{P}^{-}(\xi) \subset W^{u}\left(P_{\varepsilon}^{-}\right)$can be represented by a two-parameter family, $\gamma_{P}^{-}(\xi)=\gamma_{P}^{-}\left(\xi ; v_{*}, w_{*}\right)$, where $\left(v_{*}, w_{*}\right)$ represents the jump mid-point. Of course, we only consider the part of $W^{u}\left(P_{\varepsilon}^{-}\right)$that is spanned by orbits $\gamma_{P}^{-}(\xi)$ that are $\mathcal{O}(\varepsilon)$ close to a heteroclinic solution of the FRS (2.5) away from $\mathcal{M}_{\varepsilon}^{-}$ and $\mathcal{M}_{\varepsilon}^{+}$, i.e., we do not pay attention to the other 'half' of $W^{u}\left(P_{\varepsilon}^{-}\right)$that is spanned by solutions with a monotonically decreasing $u$-coordinate-see Fig. 2. More precisely, $\gamma_{P}^{-}(\xi)$ is exponentially close to $\mathcal{M}_{\varepsilon}^{-}$for asymptotically large, negative values of $\xi$, jumps away as $\xi$ increases, and crosses through the $\{u=0\}$ hyperplane at

$$
\gamma_{P}^{-}\left(-\xi_{P, *}\right)=\gamma_{P}^{-}\left(-\xi_{P, *}\left(v_{*}, w_{*}\right)\right)=\left(0, p_{*}, v_{*}, q_{*}, w_{*}, r_{*}\right) .
$$

Note that $\gamma_{P}^{-}\left(\xi ; v_{*}, w_{*}\right)$ must be exponentially close to the slow unstable manifold $W_{\text {slow }}^{u}\left(P_{\varepsilon}^{-}\right)$ $\subset \mathcal{M}_{\varepsilon}^{-}$that is spanned by $\ell_{v}^{u,-}$ and $\ell_{w}^{u,-}(2.12)$, so that $q_{*}=v_{*}+1, r_{*}=w_{*}+1$ as in (2.19). Moreover, we note that this family of orbits $\gamma_{P}^{-}\left(\xi ; v_{*}, w_{*}\right)$ with finite pairs $\left(v_{*}, w_{*}\right)$ has as its natural geometric completion the slow unstable manifold $W_{\text {slow }}^{u}\left(P_{\varepsilon}^{-}\right) \subset \mathcal{M}_{\varepsilon}$ in the limit that $\left|v_{*}\right| \rightarrow \infty$ and $\left|w_{*}\right| \rightarrow \infty$ such that their ratio remains fixed.

Within $W^{u}\left(P_{\varepsilon}^{-}\right)$, there is a priori a one-parameter family of orbits that is forward asymptotic to $\mathcal{M}_{\varepsilon}^{+}$, because $W^{u}\left(P_{\varepsilon}^{-}\right) \cap W^{s}\left(\mathcal{M}_{\varepsilon}^{+}\right)$is the intersection of a three- and a five-dimensional manifold in a six-dimensional space, i.e., $W^{u}\left(P_{\varepsilon}^{-}\right) \cap W^{s}\left(\mathcal{M}_{\varepsilon}^{+}\right)$is expected to be two-dimensional. The Melnikov calculus $[3,6,20]$ of the previous section implies that $\gamma_{P}^{-}(\xi$; $\left.v_{*}, w_{*}\right) \subset W^{u}\left(P_{\varepsilon}^{-}\right) \cap W^{s}\left(\mathcal{M}_{\varepsilon}^{+}\right)$if $v_{*}$ and $w_{*}$ are related by (2.17). By construction, $W^{u}\left(P_{\varepsilon}^{-}\right) \cap W^{s}\left(\mathcal{M}_{\varepsilon}^{+}\right)$is spanned by $\gamma_{\text {het }}^{-}\left(\xi ; v_{*}\right)=\gamma_{P}^{-}\left(\xi ; v_{*}, w_{*}\left(v_{*}\right)\right)$ with $w_{*}\left(v_{*}\right)$ given by (2.17).

The evolution of $\gamma_{\text {het }}^{-}\left(\xi ; v_{*}\right)$ near $\mathcal{M}_{\varepsilon}^{+}$is governed by the linear SRS (2.11). If $v_{*}, w_{*} \in$ $(-1,0)$, then $\gamma_{\text {het }}^{-}(\xi)$ intersects the $\{q=0\}$-hyperplane (Fig. 3). We may assume that the intersection $\gamma_{\text {het }}^{-}\left(\xi ; v_{*}\right) \cap\{q=0\}$ takes place at $\xi=0$. This assumption determines the jump mid-point $\xi_{\text {het }, *}\left(v_{*}\right)=\xi_{P, *}\left(v_{*}, w_{*}\left(v_{*}\right)\right)$. Moreover, it follows that $\xi_{\text {het, } *}\left(v_{*}\right)>0$ (2.26). For $\xi>-\xi_{\text {het, } *}\left(v_{*}\right)+\mathcal{O}(1 / \sqrt{\varepsilon})$, i.e., if $\gamma_{\text {het }}^{-}\left(\xi ; v_{*}\right)$ is exponentially close to $\mathcal{M}_{\varepsilon}^{+}$, the evolution of the $r$-coordinate $r_{\text {het }}^{-}\left(\xi ; v_{*}\right)$ of $\gamma_{\text {het }}^{-}\left(\xi ; v_{*}\right)$ can be computed explicitly. For general $v_{*}, r_{\text {het }}^{-}\left(0 ; v_{*}\right) \neq 0$, but there are special values of $v_{*}$ such that $r_{\text {het }}^{-}\left(0 ; v_{*}\right)=0$. In fact, $r_{\text {het }}^{-}\left(0 ; v_{*}\right)=0$ if and only if $v_{*}=-A_{0, *}^{2}$, where $A_{0, *}$ solves an algebraic equation that is to leading order given by (2.22). Note that this is in essence how (2.22) has been obtained. However, also note that the relation (2.22) has been deduced for the so far only formally constructed homoclinic orbit $\gamma_{h, j}^{-}(\xi) \subset W^{u}\left(P_{\varepsilon}^{-}\right) \cap W^{s}\left(P_{\varepsilon}^{-}\right)$, while $A_{0, *}$ corresponds to the heteroclinic orbit $\gamma_{\text {het }}^{-}\left(\xi ; v_{*}\right) \subset W^{u}\left(P_{\varepsilon}^{-}\right) \cap W^{s}\left(\mathcal{M}_{\varepsilon}^{+}\right)$. This is explained by the fact that $\xi_{j, *}$, the position of the jump mid-point of $\gamma_{h, j}^{-}(\xi)$, is of $\mathcal{O}(1 / \varepsilon)(2.23)$. Thus $\gamma_{h, j}^{-}(\xi)$ must be exponentially close to $\mathcal{M}_{\varepsilon}^{+}$for an asymptotically long 'time'. Hence, it must be exponentially close to $W^{s}\left(\mathcal{M}_{\varepsilon}^{+}\right)$. We define the (rigorously constructed) critical heteroclinic orbit $\gamma_{0, *}^{-}(\xi)$ by $\gamma_{0, *}^{-}(\xi)=\gamma_{\text {het }}^{-}\left(\xi ; v_{*}\right)$ with $v_{*}$ determined by $A_{0, *}$. Moreover, we observe that $\gamma_{0, *}^{-}(\xi)$ 
is such that $\left\|\gamma_{h, j}^{-}(\xi)-\gamma_{0, *}^{-}(\xi)\right\|$ is exponentially small for $\xi<0$; and $\left|A_{j}-A_{0, *}\right|$ is also exponentially small, but nonzero. Note that $\gamma_{0, *}^{-}(\xi)$ cannot be symmetric, since it remains exponentially close to $\mathcal{M}_{\varepsilon}^{+}$for $\xi>0$; this necessarily implies that $p_{0, *}^{-}(0) \neq 0$.

Now assume that $K \neq 0$, i.e., that there exits at least one solution $A=A_{j} \in(0,1)$ of (2.22), and that $(\alpha, \beta, \gamma, D)$ are such that $W^{u}\left(\mathcal{M}_{\varepsilon}^{-}\right)$and $W^{s}\left(\mathcal{M}_{\varepsilon}^{+}\right)$intersect transversely, i.e., that $\gamma$ is not asymptotically close to $\gamma_{c 1, c 2}(\alpha, \beta, D)$, the values at which the saddle-node bifurcations occur (2.24). The above arguments imply that the heteroclinic orbit $\gamma_{0, *}^{-}(\xi) \subset$ $W^{u}\left(P_{\varepsilon}^{-}\right) \cap W^{s}\left(\mathcal{M}_{\varepsilon}^{+}\right)$with $A_{0, *}=A_{j}$ to leading order, exists and, by construction, that $\gamma_{0, *}^{-}(0) \in\{q=r=0\}$.

By definition, the orbit $\gamma_{0, *}^{-}(\xi)$ for $\xi \in(a, b)$ spans a curve $\Gamma_{0, *}^{-}(a, b) \subset \mathbb{R}^{6}$, and there is a three-dimensional tube $\mathcal{T}_{0, *}^{-} \subset W^{u}\left(P_{\varepsilon}^{-}\right)$around $\Gamma_{0, *}^{-}(a, b)$ (for any $-\infty<a<$ $b \leq \infty)$ which consists of all orbits $\gamma^{-}\left(\xi ; v_{*}, w_{*}\right) \subset W^{u}\left(P_{\varepsilon}^{-}\right)$with $\left(v_{*} ; w_{*}\right)$ so close to $\left(-A_{0, *}^{2}, w_{*}\left(-A_{0, *}^{2}\right)\right)$ that

$$
\sup _{\xi \leq-\frac{1}{2} \xi_{0, *}}\left\|\gamma^{-}\left(\xi ; v_{*}, w_{*}\right)-\gamma_{0, *}^{-}(\xi)\right\|<e^{-\frac{1}{\sqrt{\varepsilon}}},
$$

where $-\xi_{0, *}=-\xi_{\text {het, } *}\left(v_{*}\right)$, the position of the jump mid-point of $\gamma_{0, *}^{-}(\xi)$. The existence of $\mathcal{T}_{0, *}^{-}$follows from the continuous dependence on the initial conditions of solutions of smooth ODEs (as (2.1) clearly is); $\mathcal{T}_{0, *}^{-}$defines an open neighborhood of $\Gamma_{0, *}^{-}(a, b)$ for any $-\infty<a<b \leq \infty$ in the relative topology of $W^{u}\left(P_{\varepsilon}^{-}\right)$. Note that $\mathcal{T}_{0, *}^{-}$contains both orbits that jump away from $\mathcal{M}_{\varepsilon}^{+} \mathcal{O}(\sqrt{\varepsilon})$ close to $\gamma_{0, *}^{-}\left(-\frac{1}{2} \xi_{0, *}\right)$-these are the orbits close to $\partial \mathcal{T}_{0, *}^{-}$that only remain close to $\mathcal{M}_{\varepsilon}^{+}$up to $\xi=-\frac{1}{2} \xi_{0, *}+\mathcal{O}(1 / \sqrt{\varepsilon})$-and orbits that are exponentially close to $\mathcal{M}_{\varepsilon}^{+}$for arbitrarily long 'time' - the orbits that are close enough to $\gamma_{0, *}^{-}(\xi)$. Note also that the 'secondary' jump mid-points, i.e., the points at which the orbits $\gamma^{-}\left(\xi ; v_{*}, w_{*}\right)$ take off again from $\mathcal{M}_{\varepsilon}^{+}$, of all orbits in $\mathcal{T}_{0, *}^{-}$must be exponentially close to the curve $\Gamma_{0, *}^{-}\left(-\frac{1}{2} \xi_{0, *}, \infty\right)$, that is itself exponentially close to $\mathcal{M}_{\varepsilon}^{+}$and is approximated, or represented, by a part of a solution curve of (2.11)—compare to region 3 in Fig. 4 in which the curve $\Gamma_{0, *}^{-}\left(-\xi_{*}, \xi_{*}\right)$ is approximated.

The tube $\mathcal{T}_{0, *}^{-}$is stretched by the fast dynamics near $\mathcal{M}_{\varepsilon}^{+}$into a three-dimensional manifold that is no longer exponentially small in the direction of the fast unstable eigenvalue of $\mathcal{M}_{\varepsilon}^{+}$-see Remark 2.2. In fact, $\mathcal{T}_{0, *}^{-}$is exponentially close and parallel to $W^{u}\left(\mathcal{M}_{\varepsilon}^{+}\right)$. Since $W^{u}\left(\mathcal{M}_{\varepsilon}^{+}\right)$intersects $W^{s}\left(\mathcal{M}_{\varepsilon}^{-}\right)$transversely-which can be shown by the same Melnikovtype arguments that established the intersection of $W^{u}\left(\mathcal{M}_{\varepsilon}^{-}\right)$and $W^{s}\left(\mathcal{M}_{\varepsilon}^{+}\right)$-it follows that $\mathcal{T}_{0, *}^{-} \cap W^{s}\left(\mathcal{M}_{\varepsilon}^{-}\right)$exists as a two-dimensional submanifold of $\mathcal{T}_{0, *}^{-}$. We label this manifold as $\mathcal{S}_{0, *}^{-}$; it consists of a one-parameter family of orbits $\gamma^{-}\left(\xi ; v_{*}, w_{*}\right) \subset W^{u}\left(P_{\varepsilon}^{-}\right) \cap W^{s}\left(\mathcal{M}_{\varepsilon}^{-}\right)$, i.e., orbits in $W^{u}\left(P_{\varepsilon}^{-}\right)$that are homoclinic to $\mathcal{M}_{\varepsilon}^{-}$. Since $\mathcal{T}_{0, *}^{-}$is exponentially close to $\gamma_{0, *}^{-}(\xi)$ for $\xi \leq-\frac{1}{2} \xi_{0, *}$, and since $\gamma_{0, *}^{-}(\xi)$ takes off from $\mathcal{M}_{\varepsilon}^{-}$at $W_{\text {slow }}^{u}\left(P_{\varepsilon}^{-}\right)$, it follows by the reversibility symmetry (2.2) that the orbits in $\mathcal{S}_{0, *}^{-}$touch down on $\mathcal{M}_{\varepsilon}^{-}$close to $W_{\text {slow }}^{s}\left(P_{\varepsilon}^{-}\right)$, the slow stable manifold of $P_{\varepsilon}^{-}$in $\mathcal{M}_{\varepsilon}^{-}$that is spanned by $\ell_{v, w}^{s,-}$.

The existence of the homoclinic orbit $\gamma_{h, j}^{-}(\xi)$ is established if it can be shown that there is an orbit $\gamma^{-}\left(\xi ; v_{*}, w_{*}\right) \subset \mathcal{S}_{0, *}^{-}$that indeed touches down exactly on $W_{\text {slow }}^{s}\left(P_{\varepsilon}^{-}\right)$. This result will follow from another application of the reversibility symmetry. The above construction of the two-dimensional manifold $\mathcal{S}_{0, *}^{-} \subset W^{u}\left(P_{\varepsilon}^{-}\right) \cap W^{s}\left(\mathcal{M}_{\varepsilon}^{-}\right)$, that is based on the heteroclinic orbit $\gamma_{0, *}^{-}(\xi) \subset W^{u}\left(P_{\varepsilon}^{-}\right) \cap W^{s}\left(\mathcal{M}_{\varepsilon}^{+}\right)$and on the tube $\mathcal{T}_{0, *}^{-}$, has a symmetric counterpart in 
the two-dimensional manifold $\mathcal{S}_{0, *}^{+} \subset W^{s}\left(P_{\varepsilon}^{-}\right) \cap W^{u}\left(\mathcal{M}_{\varepsilon}^{-}\right)$, that is based on the heteroclinic orbit $\gamma_{0, *}^{+}(\xi) \subset W^{s}\left(P_{\varepsilon}^{-}\right) \cap W^{u}\left(\mathcal{M}_{\varepsilon}^{+}\right)$and on the tube $\mathcal{T}_{0, *}^{+}$. Note that by construction all orbits in $\mathcal{S}_{0, *}^{+}$touch down (or: take off in backward 'time') on $W_{\text {slow }}^{s}\left(P_{\varepsilon}^{-}\right) \subset \mathcal{M}_{\varepsilon}^{-}$. Thus, $\gamma_{h, j}^{-}(\xi)$ exists if it can be shown that $\mathcal{S}_{0, *}^{-}$and $\mathcal{S}_{0, *}^{+}$intersect.

To show this, we first note that

$$
\mathcal{S}_{0, *}^{-} \cap \mathcal{S}_{0, *}^{+}=\mathcal{T}_{0, *}^{-} \cap \mathcal{T}_{0, *}^{+} \subset W^{u}\left(P_{\varepsilon}^{-}\right) \cap W^{s}\left(P_{\varepsilon}^{-}\right),
$$

since orbits in $\mathcal{T}_{0, *}^{-}$that are also in $\mathcal{T}_{0, *}^{+} \subset W^{s}\left(P_{\varepsilon}^{-}\right) \subset W^{s}\left(\mathcal{M}_{\varepsilon}^{-}\right)$must, by definition, lie inside $\mathcal{S}_{0, *}^{-}$. Moreover,

$$
\operatorname{dim}\left(\mathcal{S}_{0, *}^{-} \cap \mathcal{S}_{0, *}^{+}\right)=\operatorname{dim}\left(\mathcal{T}_{0, *}^{-} \cap \mathcal{T}_{0, *}^{+}\right)=1 .
$$

Since both $\mathcal{S}_{0, *}^{ \pm}$consist of solutions of (2.1), the dimension of $\mathcal{S}_{0, *}^{-} \cap \mathcal{S}_{0, *}^{+}$cannot be zero, i.e., $\mathcal{S}_{0, *}^{-} \cap \mathcal{S}_{0, *}^{+}$cannot be a point. It also cannot be two, which would imply that the two-dimensional sets $\mathcal{S}_{0, *}^{ \pm}$coincide. This is not the case, since $\mathcal{S}_{0, *}^{ \pm}$are, as subsets of $\mathcal{T}_{0, *}^{ \pm}$, stretched like $\mathcal{T}_{0, *}^{ \pm}$, thus $\mathcal{S}_{0, *}^{-}$is parallel to $W^{u}\left(\mathcal{M}_{\varepsilon}^{+}\right)$and $\mathcal{S}_{0, *}^{+}$to $W^{s}\left(\mathcal{M}_{\varepsilon}^{+}\right)$. Hence, we may conclude that we have proved the existence of the (locally) uniquely determined homoclinic orbit $\gamma_{h, j}(\xi) \subset W^{u}\left(P_{\varepsilon}^{-}\right) \cap W^{s}\left(P_{\varepsilon}^{-}\right)$, if we have shown that $\mathcal{T}_{0, *}^{-}$and $\mathcal{T}_{0, *}^{+}$intersect.

This follows from the local stretching of the tubes $\mathcal{T}_{0, *}^{-}$and $\mathcal{T}_{0, *}^{+}$near $\mathcal{M}_{\varepsilon}^{+}$. To see this, we consider the curves $\Gamma_{0, *}^{-}\left(-\frac{1}{2} \xi_{0, *}, \frac{1}{2} \xi_{0, *}\right)$ and $\Gamma_{0, *}^{+}\left(-\frac{1}{2} \xi_{0, *}, \frac{1}{2} \xi_{0, *}\right)$ that are associated to $\gamma_{0, *}^{-}(\xi)$ and $\gamma_{0, *}^{+}(\xi)$ (note that $\gamma_{0, *}^{+}(\xi)$ jumps at $+\xi_{0, *}$ by (2.2)). By construction, $\Gamma_{0, *}^{-}\left(-\frac{1}{2} \xi_{0, *}, \frac{1}{2} \xi_{0, *}\right)$ and $\Gamma_{0, *}^{+}\left(-\frac{1}{2} \xi_{0, *}, \frac{1}{2} \xi_{0, *}\right)$ are exponentially close to each other and exponentially close to $\mathcal{M}_{\varepsilon}^{+}$. The tube $\mathcal{T}_{0, *}^{-}$is stretched in the direction of the fast unstable eigenvalue of $\mathcal{M}_{\varepsilon}^{+}$near $\Gamma_{0, *}^{ \pm}\left(-\frac{1}{2} \xi_{0, *}, \frac{1}{2} \xi_{0, *}\right)$ and is exponentially close to $W^{u}\left(\mathcal{M}_{\varepsilon}^{+}\right)$, while $\mathcal{T}_{0, *}^{+}$ is stretched in the direction of the fast stable eigenvalue of $\mathcal{M}_{\varepsilon}^{+}$near $\Gamma_{0, *}^{ \pm}\left(-\frac{1}{2} \xi_{0, *}, \frac{1}{2} \xi_{0, *}\right)$ and is exponentially close to $W^{u}\left(\mathcal{M}_{\varepsilon}^{+}\right)$. Moreover, both three-dimensional manifolds $\mathcal{T}_{0, *}^{ \pm}$ extend to two sides $-\{u<1\}$ and $\{u>1\}-$ of $\mathcal{M}_{\varepsilon}^{+}$near $\Gamma_{0, *}^{ \pm}\left(-\frac{1}{2} \xi_{0, *}, \frac{1}{2} \xi_{0, *}\right)$, since they both contain orbits that are asymptotic to $\mathcal{M}_{\varepsilon}^{+}$. Thus, $\mathcal{T}_{0, *}^{-}$and $\mathcal{T}_{0, *}^{+}$must have a nontrivial intersection. This completes the proof for $K>0$.

Observe that the left hand side of (2.22) has at most one extremum for $A \in(0,1)$, namely

$$
A=\left(-\frac{\alpha D}{\beta}\right)^{-\frac{1}{2} \frac{D}{D-1}},
$$

see (2.25). Therefore, $K$ cannot be more than two.

Finally, we briefly consider the situation in which $K=0$, i.e., in which there is no solution $A \in(0,1)$ of (2.22). In this case, the critical heteroclinic orbits $\gamma_{0, *}^{\mp}(\xi)$ cannot be constructed, and it follows immediately that $W^{u}\left(P_{\varepsilon}^{-}\right) \cap W^{s}\left(P_{\varepsilon}^{-}\right)=\emptyset$. The saddle-node bifurcations occur at the transition from $K=2$ to $K=0$ and must be locally unique by the $C^{1}$-smoothness with respect to $\varepsilon$ of the stable and unstable manifolds of $\mathcal{M}_{\varepsilon}^{ \pm}$and $P_{\varepsilon}^{ \pm}[8,9]$.

Remark 2.2 In $[12,13]$, the stretching and squeezing associated to the passage of an invariant manifold along a slow manifold are described by the Exchange Lemma. This lemma can be used to study the deformation of $W^{u}\left(P_{\varepsilon}^{-}\right)$as it passes along $\mathcal{M}_{\varepsilon}^{+}$. Indeed, one may verify explicitly that the sets of touch down points of the tracked manifold on the slow manifolds are transverse to the flows on those manifolds. However, we have chosen for a somewhat more direct approach here. 
2.5 Explicit Analysis of the Number $K$ of Stationary One-Pulse Solutions

Theorem 2.1 above establishes that $K \leq 2$. In this section, we carry out a straightforward analysis of the jump condition (2.22) to derive explicit results for the number $(K)$ of stationary one-pulse solutions in (1.6) for a given set of parameters. The following lemma is an example; it is stated without proof.

Lemma 2.1 Let $(\alpha, \beta, \gamma, D, \tau, \theta, \varepsilon)$ be such that $|\alpha D|>|\beta|$. Then, for $\varepsilon>0$ small enough, and $\gamma_{c 1, c 2}$ as given in (2.24), we have

(a1) if $\operatorname{sgn}(\alpha)=\operatorname{sgn}(\beta), \operatorname{sgn}(\gamma)=\operatorname{sgn}(\alpha)$, and $|\gamma|<|\alpha+\beta|$, then $K=1$.

(a2) if $\operatorname{sgn}(\alpha)=\operatorname{sgn}(\beta), \operatorname{sgn}(\gamma)=\operatorname{sgn}(\alpha)$, and $|\gamma|>|\alpha+\beta|$, then $K=0$.

(a3) if $\operatorname{sgn}(\alpha)=\operatorname{sgn}(\beta)$ and $\operatorname{sgn}(\gamma) \neq \operatorname{sgn}(\alpha)$, then $K=0$.

(b1) if $\operatorname{sgn}(\alpha)=-1=-\operatorname{sgn}(\beta), \alpha+\beta>0$, and $\operatorname{sgn}(\gamma)=-1$, then $K=0$.

(b2) if $\operatorname{sgn}(\alpha)=-1=-\operatorname{sgn}(\beta), \alpha+\beta>0$, and $0<\gamma<\alpha+\beta$, then $K=1$.

(b3) if $\operatorname{sgn}(\alpha)=-1=-\operatorname{sgn}(\beta), \alpha+\beta>0$, and $\alpha+\beta<\gamma<\gamma_{c 1}$, then $K=2$.

(b4) if $\operatorname{sgn}(\alpha)=-1=-\operatorname{sgn}(\beta), \alpha+\beta>0$, and $\gamma>\gamma_{c 1}$, then $K=0$.

(c1) if $\operatorname{sgn}(\alpha)=-1=-\operatorname{sgn}(\beta), \alpha+\beta<0$, and $\gamma<\alpha+\beta$, then $K=0$.

(c2) if $\operatorname{sgn}(\alpha)=-1=-\operatorname{sgn}(\beta), \alpha+\beta<0$, and $\alpha+\beta<\gamma<0$, then $K=1$.

(c3) if $\operatorname{sgn}(\alpha)=-1=-\operatorname{sgn}(\beta), \alpha+\beta<0$, and $0<\gamma<\gamma_{c 1}$, then $K=2$.

(c4) if $\operatorname{sgn}(\alpha)=-1=-\operatorname{sgn}(\beta), \alpha+\beta<0$, and $\gamma>\gamma_{c 1}$, then $K=0$.

(d1) if $\operatorname{sgn}(\alpha)=1=-\operatorname{sgn}(\beta), \alpha+\beta>0$, and $\gamma<\gamma_{c 2}$, then $K=0$.

(d2) if $\operatorname{sgn}(\alpha)=1=-\operatorname{sgn}(\beta), \alpha+\beta>0$, and $\gamma_{c 2}<\gamma<0$, then $K=2$.

(d3) if $\operatorname{sgn}(\alpha)=1=-\operatorname{sgn}(\beta), \alpha+\beta>0$, and $0<\gamma<\alpha+\beta$, then $K=1$.

(d4) if $\operatorname{sgn}(\alpha)=1=-\operatorname{sgn}(\beta), \alpha+\beta>0$, and $\gamma>\alpha+\beta$, then $K=0$.

(e1) if $\operatorname{sgn}(\alpha)=1=-\operatorname{sgn}(\beta), \alpha+\beta<0$, and $\gamma<\gamma_{c 2}$, then $K=0$.

(e2) if $\operatorname{sgn}(\alpha)=1=-\operatorname{sgn}(\beta), \alpha+\beta<0$, and $\gamma_{c 2}<\gamma<\alpha+\beta$, then $K=2$.

(e3) if $\operatorname{sgn}(\alpha)=1=-\operatorname{sgn}(\beta), \alpha+\beta<0$, and $\alpha+\beta<\gamma<0$, then $K=1$.

(e4) if $\operatorname{sgn}(\alpha)=1=-\operatorname{sgn}(\beta), \alpha+\beta<0$, and $\gamma>0$, then $K=0$.

See also Fig. 5, where we plotted (2.22) for certain parameter combinations. The left frame represents the cases (b1)-(b4), the right frame (d1)-(d4).

\section{Travelling Pulse Solutions}

In this section, we establish the existence of localized one-pulse solutions to (1.6) that travel with a fixed, well-determined, speed. As in the previous section, we will construct these pulses as homoclinic orbits $\gamma_{t r, j}^{-}(\xi)$ to the critical point $P_{\varepsilon}^{-}$.

3.1 The Formal Construction of Travelling One-Pulse Solutions, $\gamma_{t r, j}^{-}(\xi)$

We introduce the moving coordinates $\eta=x-\varepsilon^{2} c t$ and, with a slight abuse of notation, set $\xi=\eta / \varepsilon$, so that (1.6) reduces to the six-dimensional dynamical system,

$$
\left\{\begin{aligned}
u_{\xi} & =p \\
p_{\xi} & =-u+u^{3}+\varepsilon(\alpha v+\beta w+\gamma-c p) \\
v_{\xi} & =\varepsilon q \\
q_{\xi} & =\varepsilon(v-u)-\varepsilon^{3} c \tau q \\
w_{\xi} & =\frac{\varepsilon}{D} r \\
r_{\xi} & =\frac{\varepsilon}{D}(w-u)-\frac{\varepsilon^{3}}{D^{2}} c \theta r,
\end{aligned}\right.
$$


with an additional parameter $c$ for the speed of the travelling pulse. The structure of this equation justifies our choice for the magnitude of $c\left(=\mathcal{O}\left(\varepsilon^{2}\right)\right)$. With this scaling, the perturbation of the fast $(u, p)$-subsystem induced by $c$ is of the same order as the perturbations induced by the $V, W$-components in the $U$-equation of (1.6). Note that, unlike (2.1), (3.1) depends explicitly on the parameters $\tau$ and $\theta$. However, the critical points of (3.1) are identical to those of (2.1) and, thus, given by (2.4).

The fast reduced system is identical to (2.5), as long as $\tau, \theta \ll \frac{1}{\varepsilon^{3}}$, and is thus again governed by the Hamiltonian $H(u, p)$ (2.6). For any $c$ of $\mathcal{O}(1)$, system (3.1) possesses two invariant slow manifolds and their associated stable and unstable manifolds, which we denote, with a slight abuse of notation, by $\mathcal{M}_{\varepsilon}^{ \pm}$and $W^{s, u}\left(\mathcal{M}_{\varepsilon}^{ \pm}\right)$. Although $\mathcal{M}_{\varepsilon}^{ \pm}$depend on $c$, the leading and first order approximations of $\mathcal{M}_{\varepsilon}^{ \pm}$are still given by (2.8) and (2.9), so that it again follows that $\left.H(u, p)\right|_{\mathcal{M}_{\varepsilon}^{ \pm}}=\mathcal{O}\left(\varepsilon^{2}\right)(2.16)$.

However, there are two significant differences between (3.1) and (2.1). First, (3.1) does not have the reversibility symmetry of (2.1) for $c \neq 0$. As a consequence, we cannot expect to find symmetric pulses and, more importantly, we cannot exploit the symmetry in the construction of the pulse and in the associated validity proof. However, system (3.1) does inherit the symmetry,

$$
\xi \rightarrow-\xi, p \rightarrow-p, q \rightarrow-q, r \rightarrow-r \text { and } c \rightarrow-c
$$

which implies that the travelling pulses do not have a preferred direction, i.e., to any pulse travelling with speed $c>0$, there is a symmetrical counterpart that travels with speed $c<0$. Second,

$$
\frac{d}{d \xi} H(u(\xi), p(\xi))=\varepsilon p(\alpha v+\beta w+\gamma-c p),
$$

instead of (2.14), which implies that the Melnikov conditions will depend in an $\mathcal{O}(1)$ fashion on $c$-which also further validates our scaling of the magnitude of the speed of the pulses.

As in Sect. 2.2, we define the position of the jump mid-points of $\gamma_{t r, j}^{-}(\xi)$ to be $\mp \xi_{*}$, i.e., $\gamma_{t r, j}^{-}(\xi)$ crosses the hyperplane $\{u=0\}$ at $\xi=\mp \xi_{*}\left(\xi_{*}>0\right)$. The coordinates of the jump mid-points are defined by

$$
\gamma_{t r, j}^{-}\left(\mp \xi_{*}\right)=\left(0, p_{*}^{\mp}, v_{*}^{\mp}, q_{*}^{\mp}, w_{*}^{\mp}, r_{*}^{\mp}\right) .
$$

Unlike the symmetric stationary case, the coordinates of the jump through the fast field from $\mathcal{M}_{\varepsilon}^{-}$to $\mathcal{M}_{\varepsilon}^{+}$, denoted by $\left(p_{*}^{-}, v_{*}^{-}, q_{*}^{-}, w_{*}^{-}, r_{*}^{-}\right)$, will differ from those of the jump back from $\mathcal{M}_{\varepsilon}^{+}$to $\mathcal{M}_{\varepsilon}^{-}$, denoted by $\left(p_{*}^{+}, v_{*}^{+}, q_{*}^{+}, w_{*}^{+}, r_{*}^{+}\right)$. Moreover, the middle of the pulse, $\gamma_{t r, j}^{-}(0)$, has become slightly artificial by this definition, in the sense that $\xi=0$ does not in general correspond to an extremum of any of the $U-, V$ - or $W$-components in (1.6). Nevertheless, with this definition we can use the same partition of the homoclinic orbit $\gamma_{t r, j}^{-}(\xi)$ into five regions - see Sect. 2.2-with $I_{f, s}^{\mp}$ and $I_{s}^{0}$ as in (2.13).

We again use the Melnikov function to measure the distance between $W^{u}\left(\mathcal{M}_{\varepsilon}^{-}\right)$and $W^{s}\left(\mathcal{M}_{\varepsilon}^{+}\right)$. We find, assuming that $\xi_{*}=\mathcal{O}\left(\frac{1}{\varepsilon}\right)$, 


$$
\begin{aligned}
\Delta_{f}^{-} H\left(v_{*}^{-}, q_{*}^{-}, w_{*}^{-}, r_{*}^{-}\right)= & \int_{I_{f}^{-}} H_{\xi} d \xi \\
= & \int_{I_{f}^{-}} \varepsilon p_{h}^{0,-}\left(\xi+\xi_{*}\right)\left(\alpha v_{*}^{-}+\beta w_{*}^{-}+\gamma-c p_{h}^{0,-}\left(\xi+\xi_{*}\right)\right) d \xi \\
& +\mathcal{O}(\varepsilon \sqrt{\varepsilon}) \\
= & 2 \varepsilon\left(\alpha v_{*}^{-}+\beta w_{*}^{-}+\gamma-\frac{1}{3} \sqrt{2} c\right)+\mathcal{O}(\varepsilon \sqrt{\varepsilon}),
\end{aligned}
$$

where we have implicitly used that the slow coordinates $(v, p, w, r)$ do not vary to leading order during a jump through the fast field, i.e., that

$$
\Delta_{f}^{\mp} v, \Delta_{f}^{\mp} p, \Delta_{f}^{\mp} w, \Delta_{f}^{\mp} q=\mathcal{O}(\sqrt{\varepsilon}),
$$

(see 2.15). Since $\left.H(u, p)\right|_{\mathcal{M}_{\varepsilon}^{\mp}}=\mathcal{O}\left(\varepsilon^{2}\right)$, we find as the first Melnikov condition,

$$
\alpha v_{*}^{-}+\beta w_{*}^{-}+\gamma=\frac{1}{3} \sqrt{2} c .
$$

Since there is no reversibility symmetry, the second Melnikov condition for the jump from $\mathcal{M}_{\varepsilon}^{+}$to $\mathcal{M}_{\varepsilon}^{-}$is slightly different,

$$
\alpha v_{*}^{+}+\beta w_{*}^{+}+\gamma=-\frac{1}{3} \sqrt{2} c
$$

which follows from

$$
\begin{aligned}
\Delta_{f}^{+} H\left(v_{*}^{+}, q_{*}^{+}, w_{*}^{+}, r_{*}^{+}\right)= & \int_{I_{f}^{+}} H_{\xi} d \xi \\
= & \int_{I_{f}^{+}} \varepsilon p_{h}^{0,+}\left(\xi+\xi_{*}\right)\left(\alpha v_{*}^{+}+\beta w_{*}^{+}+\gamma-c p_{h}^{0,+}\left(\xi+\xi_{*}\right)\right) d \xi \\
& +\mathcal{O}(\varepsilon \sqrt{\varepsilon}) \\
= & 2 \varepsilon\left(\alpha v_{*}^{+}+\beta w_{*}^{+}+\gamma+\frac{1}{3} \sqrt{2} c\right)+\mathcal{O}(\varepsilon \sqrt{\varepsilon}),
\end{aligned}
$$

(compare $p_{h}^{0,+}(\xi)$ to $\left.p_{h}^{0,-}(\xi)-(2.7)\right)$. Note that the jump conditions are consistent with the symmetry (3.2).

We can proceed (formally) as in the stationary case. We solve the (linear) slow subsystems explicitly, imposing boundary conditions like those in (2.18) at the boundaries of the three slow regions (1,3, and 5) and also imposing the Melnikov conditions (3.6) and (3.7). Here, we present this analysis for the critical case $\tau, \theta=\mathcal{O}\left(\frac{1}{\varepsilon^{2}}\right)$, since travelling pulses can only exist for these values of $\tau$ and $\theta$. More precisely, if both $\tau, \theta \ll \frac{1}{\varepsilon^{2}}$, then the flows on $\mathcal{M}_{\varepsilon}^{ \pm}$ are symmetric to leading order and the only asymmetries in the construction of $\gamma_{t r, j}^{-}(\xi)$ are introduced by the $c$ 's in the Melnikov conditions (3.6) and (3.7). From this, it follows that $c=0$, i.e., that $\gamma_{t r, j}^{-}(\xi)=\gamma_{h, j}^{-}(\xi)$, the stationary pulse-see Remark 3.1.

Thus, we introduce $\hat{\tau}$ and $\hat{\theta}$ by

$$
\hat{\tau}=\varepsilon^{2} \tau \ll \frac{1}{\varepsilon}, \quad \hat{\theta}=\varepsilon^{2} \theta \ll \frac{1}{\varepsilon} .
$$



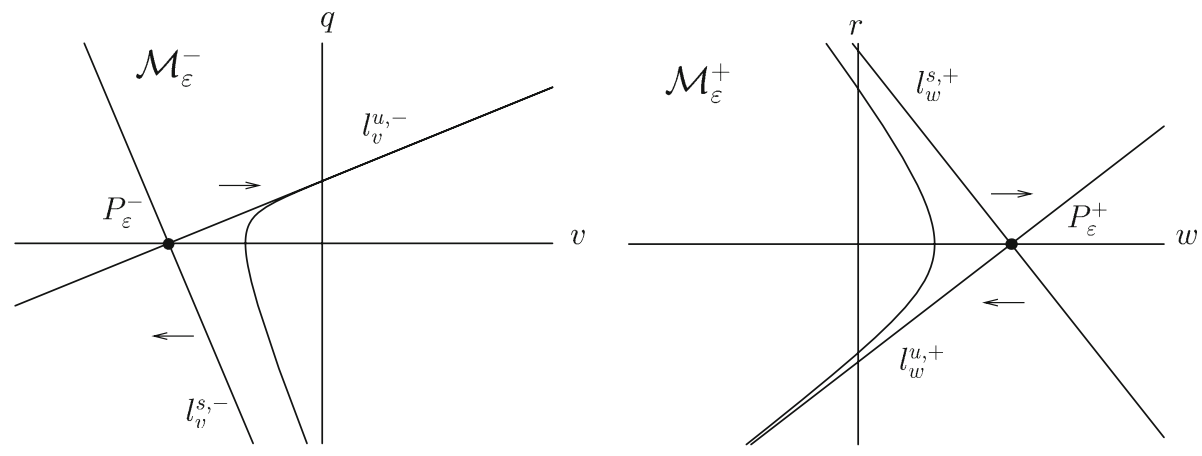

Fig. 6 The asymmetric slow flows for the $(v, q)$-subsystem on $\mathcal{M}_{\varepsilon}^{-}$(left) and the $(w, r)$-subsystem on $\mathcal{M}_{\varepsilon}^{+}$ (right) for $c$ positive

The flows on $\mathcal{M}_{\varepsilon}^{-}$and $\mathcal{M}_{\varepsilon}^{+}$are, up to correction terms of $\mathcal{O}\left(\varepsilon^{3}\right)$, given by

$$
\left\{\begin{array} { c } 
{ v _ { \xi \xi } = - \varepsilon c \hat { \tau } v _ { \xi } + \varepsilon ^ { 2 } ( v + 1 ) , } \\
{ w _ { \xi \xi } = - \varepsilon c \frac { \hat { \theta } } { D ^ { 2 } } w _ { \xi } + \frac { \varepsilon ^ { 2 } } { D ^ { 2 } } ( w + 1 ) , }
\end{array} \quad \text { and } \quad \left\{\begin{array}{c}
v_{\xi \xi}=-\varepsilon c \hat{\tau} v_{\xi}+\varepsilon^{2}(v-1), \\
w_{\xi \xi}=-\varepsilon c \frac{\hat{\theta}}{D^{2}} w_{\xi}+\frac{\varepsilon^{2}}{D^{2}}(w-1),
\end{array}\right.\right.
$$

see Fig. 6. The eigenvalues $\lambda_{v, w}^{ \pm}$of the decoupled $(v, q)$ - and $(w, r)$-subsystems are given by

$$
\lambda_{v}^{ \pm}=\frac{1}{2}\left(-c \hat{\tau} \pm \sqrt{c^{2} \hat{\tau}^{2}+4}\right), \quad \lambda_{w}^{ \pm}=\frac{1}{2} \frac{1}{D}\left(-\frac{c \hat{\theta}}{D} \pm \sqrt{\frac{c^{2} \hat{\theta}^{2}}{D^{2}}+4}\right)
$$

which clearly establishes the asymmetric character of the flows on $\mathcal{M}_{\varepsilon}^{ \pm}$(for $\hat{\tau}, \hat{\theta} \neq 0$ ). The stable and unstable manifolds of $P_{\varepsilon}^{ \pm}$restricted to $\mathcal{M}_{\varepsilon}^{ \pm}$are spanned by

$$
\begin{array}{ll}
\ell_{v}^{u, \pm}=\left\{q=\lambda_{v}^{+}(\mp 1+v)\right\}, & \ell_{w}^{u, \pm}=\left\{r=D \lambda_{w}^{+}(\mp 1+w)\right\}, \\
\ell_{v}^{s, \pm}=\left\{q=\lambda_{v}^{-}(\mp 1+v)\right\}, & \ell_{w}^{s, \pm}=\left\{r=D \lambda_{w}^{-}(\mp 1+w)\right\},
\end{array}
$$

(compare with (2.12)).

Since the slow $(v, q, w, r)$-coordinates do not vary to leading order during a jump through the fast field (3.5), we can 'match' the solutions in the slow regions 1, 3, and 5 by imposing boundary conditions as in (2.18). As in the stationary case, there are more boundary conditions than free parameters. Hence, there are relations between the coordinates of the jump mid-points,

$$
\left(v_{*}^{-}, q_{*}^{-}\right) \in \ell_{v}^{u,-},\left(w_{*}^{-}, r_{*}^{-}\right) \in \ell_{w}^{u,-},\left(v_{*}^{+}, q_{*}^{+}\right) \in \ell_{v}^{s,-},\left(w_{*}^{+}, r_{*}^{+}\right) \in \ell_{w}^{s,-},
$$

as may be seen from the system geometry (see Fig. 7). Furthermore,

$$
v_{*}^{ \pm}=s_{v}^{ \pm}\left(e^{ \pm 2 \varepsilon \lambda_{v}^{\mp} \xi_{*}}-1\right)-1, w_{*}^{ \pm}=s_{w}^{ \pm}\left(e^{ \pm 2 \varepsilon \lambda_{w}^{\mp} \xi_{*}}-1\right)-1,
$$

with

$$
s_{v}^{ \pm}=-\frac{2 \lambda_{v}^{ \pm}}{\lambda_{v}^{ \pm}-\lambda_{v}^{\mp}}<0, \quad s_{w}^{ \pm}=-\frac{2 \lambda_{w}^{ \pm}}{\lambda_{w}^{ \pm}-\lambda_{w}^{\mp}}<0 .
$$

(Note that (3.10) and (3.11) reduce to their stationary equivalents (2.19) and (2.20) if either $c=0$ or $\hat{\tau}=\hat{\theta}=0-$ see $\operatorname{Remark} 3.1$.) We conclude that for any given pair $\left(c, \xi_{*}\right)$, the (slow) 


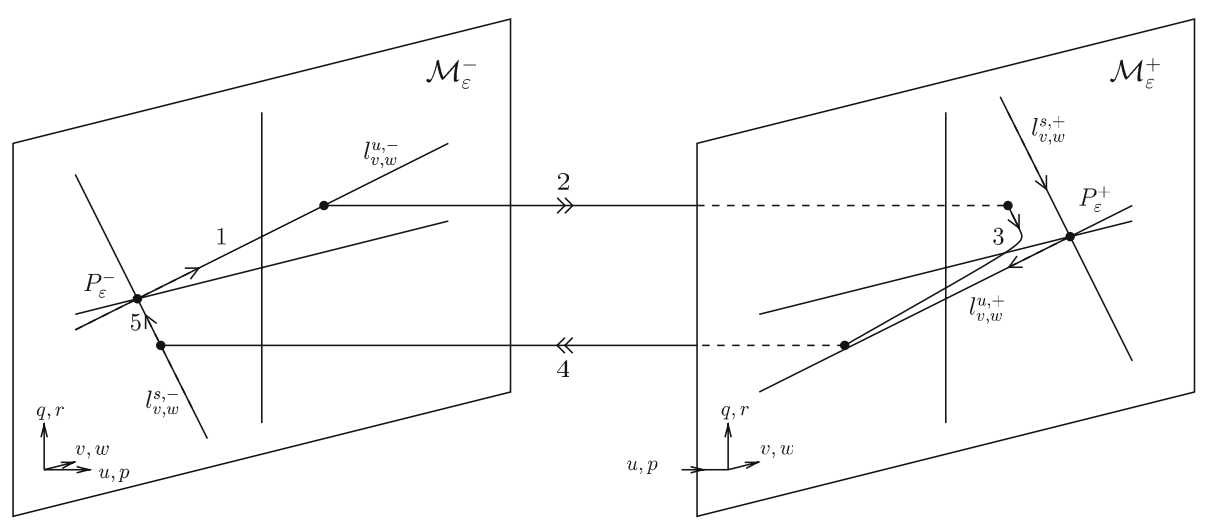

Fig. 7 A schematic sketch of a travelling pulse $\gamma_{t r, j}^{-}(\xi)$ homoclinic to $P_{\varepsilon}^{-}$

coordinates $\left(v_{*}^{\mp}, q_{*}^{\mp}, w_{*}^{\mp}, r_{*}^{\mp}\right)$ of the jump mid-points are uniquely determined by the above conditions combined with the matching conditions (3.5). Moreover, we have the following leading order approximations of the $v$ - and $w$-components of $\gamma_{t r, j}^{-}(\xi)$ in the slow regions $(1$, $3,5)$,

$$
\begin{array}{r}
v_{t r}=\left\{\begin{array}{lr}
-2 s_{v}^{-} e^{\varepsilon \lambda_{v}^{+} \xi} \sinh \varepsilon \lambda_{v}^{+} \xi_{*}-1 & \text { in } 1, \\
s_{v}^{-} e^{\varepsilon \lambda_{v}^{+}\left(\xi-\xi_{*}\right)}+s_{v}^{+} e^{\varepsilon \lambda_{v}^{-}\left(\xi+\xi_{*}\right)}+1 & \text { in 3, } \\
2 s_{v}^{+} e^{\varepsilon \lambda_{v}^{-} \xi} \sinh \varepsilon \lambda_{v}^{-} \xi_{*}-1 & \text { in 5, }
\end{array}\right. \\
w_{t r}=\left\{\begin{array}{lr}
-2 s_{w}^{-} e^{\varepsilon \lambda_{w}^{+} \xi} \sinh \varepsilon \lambda_{w}^{+} \xi_{*}-1 & \text { in } 1, \\
s_{w}^{-} e^{\varepsilon \lambda_{w}^{+}\left(\xi-\xi_{*}\right)}+s_{w}^{+} e^{\varepsilon \lambda_{w}^{-}\left(\xi+\xi_{*}\right)}+1 & \text { in 3, } \\
2 s_{w}^{+} e^{\varepsilon \lambda_{w}^{-} \xi} \sinh \varepsilon \lambda_{w}^{-} \xi_{*}-1 & \text { in 5, }
\end{array}\right.
\end{array}
$$

see Fig. 7. The Melnikov conditions (3.6) and (3.7) impose two relations between $c$ and $\xi_{*}$,

$$
\left\{\begin{aligned}
\frac{1}{3} \sqrt{2} c & =\alpha\left(s_{v}^{-}\left(e^{-2 \varepsilon \lambda_{v}^{+} \xi_{*}}-1\right)-1\right)+\beta\left(s_{w}^{-}\left(e^{-2 \varepsilon \lambda_{w}^{+} \xi_{*}}-1\right)-1\right)+\gamma \\
-\frac{1}{3} \sqrt{2} c & =\alpha\left(s_{v}^{+}\left(e^{2 \varepsilon \lambda_{v}^{-} \xi_{*}}-1\right)-1\right)+\beta\left(s_{w}^{+}\left(e^{2 \varepsilon \lambda_{w}^{-} \xi_{*}}-1\right)-1\right)+\gamma
\end{aligned}\right.
$$

A pair of solutions $\left(c, \xi_{*}\right)$ to (3.13) with $c \neq 0$ corresponds formally to a homoclinic solution $\gamma_{t r, j}^{-}(\xi)$ of (3.1) and thus to a pulse solution of (1.6) that travels with speed $\varepsilon^{2} c$.

Remark 3.1 If $\tau, \theta \ll \frac{1}{\varepsilon^{2}}$, i.e., if $\hat{\tau}, \hat{\theta}=0$ to leading order, then $\lambda_{v}^{ \pm}= \pm 1, \lambda_{w}^{ \pm}= \pm \frac{1}{D}$, and $s_{v}^{ \pm}=s_{w}^{ \pm}=-1$, so that (3.13) reduces to

$$
-\frac{1}{3} \sqrt{2} c=\alpha A^{2}+\beta A^{\frac{2}{D}}-\gamma=\frac{1}{3} \sqrt{2} c,
$$

to leading order, with $A$ as in (2.20). Hence, $c=0$ and $\gamma_{t r, j}^{-}(\xi)=\gamma_{h, j}^{-}(\xi)$ (2.22).

\subsection{Existence Theorem for Travelling Pulse Solutions}

Theorem 3.1 Let $(\alpha, \beta, \gamma, D, \tau, \theta, \varepsilon)$ be such that $\tau=\frac{\hat{\tau}}{\varepsilon^{2}}, \theta=\frac{\hat{\theta}}{\varepsilon^{2}}$, and assume that (3.13) has $K$ solution pairs $\left(c_{j},\left(\xi_{*}\right)_{j}\right)$ with $c_{j} \neq 0$. Let $\varepsilon>0$ be small enough. If $K=0$, then there are no homoclinic orbits to $P_{\varepsilon}^{-}$in (3.1) with $c \neq 0$. If $K>0$, there are $K$ homoclinic 
orbits $\gamma_{t r, j}^{-}(\xi), j \in\{1, \ldots, K\}$, to $P_{\varepsilon}^{-}$in (3.1) that have a structure as sketched in Fig. 7 and that correspond to travelling one-pulse solutions of (1.6) which travel with speed $\varepsilon^{2} c_{j}^{*} \neq 0$, where $c_{j}^{*}=c_{j}^{*}(\varepsilon)=c_{j}+\mathcal{O}(\varepsilon)$.

The proof of Theorem 3.1 is similar to that of Theorem 2.1 in Sect. 2.4. Nevertheless, there are differences, especially since the proof of Theorem 2.1 strongly depended on the reversibility symmetry in (2.1). The proof is given in Sect. 3.3.

Generically, $K$ can be expected to be positive for open regions in the $(\alpha, \beta, \gamma, D, \hat{\tau}, \hat{\theta})$ parameter space. However, a priori, it is not clear whether parameter combinations exist for which $K$ can be non-zero. In fact, though (3.13) is a relatively simple expression, it can-of course-not be solved explicitly. Nevertheless, it can be evaluated, and the (open) region in parameter space in which $K \neq 0$ can be determined with a simple and reliable numerical procedure. Moreover, (3.13) can be approximated asymptotically in various limit settings. As an example, we consider the case

$$
\hat{\tau}=\frac{1}{\delta} \gg 1, \hat{\theta}=h \delta \ll 1,
$$

i.e., we assume that $\hat{\tau}$ is large and $\hat{\theta}$ is small, but both still $\mathcal{O}(1)$ with respect to $\varepsilon$. We thus introduce an artificial second asymptotic parameter $\delta$ that is independent of $\varepsilon$ such that $0<\varepsilon \ll \delta \ll 1$. We further assume that all other parameters, including $h$, are $\mathcal{O}(1)$ with respect to $\delta$. We search for solutions $\left(c, \xi_{*}\right)$ of (3.13) such that

$$
c>0, c=\mathcal{O}(1), X_{*}=\varepsilon \delta \xi_{*}=\mathcal{O}(1),
$$

with respect to $\delta$. Note that this implies that we look for homoclinic orbits that spend a long 'time' $\left(\mathcal{O}\left(\frac{1}{\varepsilon \delta}\right)\right)$ near $\mathcal{M}_{\varepsilon}^{+}$. It follows by a straightforward computation from (3.11) that,

$$
v_{*}^{-}=-2 e^{2 \frac{X_{*}}{c}(1+\mathcal{O}(\delta))}+1+\mathcal{O}(\delta), v_{*}^{+}=-1+\mathcal{O}(\delta), w_{*}^{-}=\mathcal{O}(\delta), w_{*}^{+}=\mathcal{O}(\delta),
$$

so that (3.13) reduces to

$$
\frac{1}{3} \sqrt{2} c=\alpha v_{*}^{-}+\gamma+\mathcal{O}(\delta),-\frac{1}{3} \sqrt{2} c=-\alpha+\gamma+\mathcal{O}(\delta) .
$$

Hence, there exists a homoclinic orbit $\gamma_{t r, 1}^{-}(\xi)$ to $P_{\varepsilon}^{-}$in (3.1) for $\alpha>\gamma$ with

$$
c=c_{1}=\frac{3}{2} \sqrt{2}(\alpha-\gamma)+\mathcal{O}(\delta, \varepsilon) .
$$

Moreover, $X_{*, 1}$, and thus $\left(\xi_{*}\right)_{1}$, can be determined through $v_{*}^{-}$and (3.14). By the symmetry (3.2), we conclude that $K=2$ for $\hat{\tau} \gg 1, \hat{\theta} \ll 1$ and $\alpha>\gamma$.

\subsection{Proof of Theorem 3.1}

The construction of

$$
\gamma_{t r, j}^{-}(\xi) \subset W^{u}\left(P_{\varepsilon}^{-}\right) \cap W^{s}\left(P_{\varepsilon}^{-}\right) \subset W^{u}\left(P_{\varepsilon}^{-}\right) \cap W^{s}\left(\mathcal{M}_{\varepsilon}^{-}\right)
$$

is again based on a special heteroclinic orbit $\gamma_{*, *}^{-}(\xi) \subset W^{u}\left(P_{\varepsilon}^{-}\right) \cap W^{s}\left(\mathcal{M}_{\varepsilon}^{+}\right)$, a tube $\mathcal{T}_{*, *}^{-} \subset$ $W^{u}\left(P_{\varepsilon}^{-}\right)$around it, their counterparts in backwards 'time' $\gamma_{*, *}^{+}(\xi) \subset W^{s}\left(P_{\varepsilon}^{-}\right) \cap W^{u}\left(\mathcal{M}_{\varepsilon}^{+}\right)$ and $\mathcal{T}_{*, *}^{+} \subset W^{S}\left(P_{\varepsilon}^{-}\right)$, so that $\gamma_{t r, j}^{-}(\xi) \subset \mathcal{T}_{*, *}^{-} \cap \mathcal{T}_{*, *}^{+}$. 

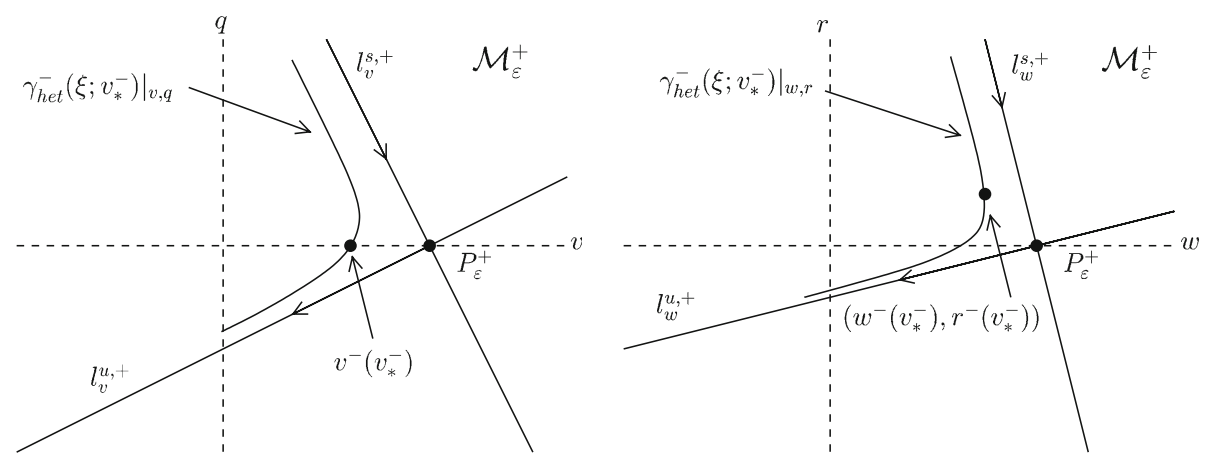

Fig. 8 Example of the construction of $v^{-}\left(v_{*}^{-}\right), w^{-}\left(v_{*}^{-}\right)$, and $r^{-}\left(v_{*}^{-}\right)$

For any $c>0$ (fixed), $W^{u}\left(P_{\varepsilon}^{-}\right)$is represented by the two-parameter family of orbits $\gamma_{P}^{-}\left(\xi ; v_{*}^{-}, w_{*}^{-}\right) \subset W^{u}\left(P_{\varepsilon}^{-}\right)$. We know by the Melnikov analysis that there is a one-parameter subfamily of orbits $\gamma_{\text {het }}^{-}\left(\xi ; v_{*}^{-}\right)=\gamma_{P}^{-}\left(\xi ; v_{*}^{-} ; w_{*}^{-}\left(v_{*}^{-}\right)\right) \subset W^{u}\left(P_{\varepsilon}^{-}\right) \cap W^{s}\left(\mathcal{M}_{\varepsilon}^{+}\right)$, with $w_{*}^{-}\left(v_{*}^{-}\right)$determined by (3.6). The orbits $\gamma_{\text {het }}^{-}\left(\xi ; v_{*}^{-}\right)$follow the slow flow on $\mathcal{M}_{\varepsilon}^{+}$, and it can be checked that those with $v_{*}^{-} \in\left(-1, S_{v}^{-}\right)$again cross the $\{q=0\}$-hyperplane. Here, $S_{v}^{-}$is determined by the observation that $\left(v_{*}^{-}, q_{*}^{-}\right) \in l_{v}^{u,-}$ in the $(v, q)$-subsystem on $\mathcal{M}_{\varepsilon}^{-}(3.10)$, while $\left(v_{*}^{-}, q_{*}^{-}\right)$must be to the left of $l_{v}^{s,+}$ in the $(v, q)$-subsystem on $\mathcal{M}_{\varepsilon}^{+}$so that $\gamma_{\text {het }}^{-}\left(\xi ; v_{*}^{-}\right)$ may cross through $\{q=0\}$; a similar condition must hold for $\left(w_{*}^{-}\left(v_{*}^{-}\right), r_{*}^{-}\right)$in the $(w, r)$ subflows on $\mathcal{M}_{\varepsilon}^{\mp}-$ see Fig. 7. For each $v_{*}^{-} \in\left(-1, S_{v}^{-}\right)$the intersection of $\gamma_{\text {het }}^{-}\left(\xi ; v_{*}^{-}\right)$with $\{q=0\}$ occurs by definition at $\xi=\xi_{\text {het }}^{-}\left(v_{*}^{-}\right) \in\left(-\xi_{*}, \xi_{*}\right)$, and these intersections define a one-dimensional curve denoted by

$$
\begin{aligned}
Z^{-} & =\left\{\left(u^{-}\left(v_{*}^{-}\right), p^{-}\left(v_{*}^{-}\right), v^{-}\left(v_{*}^{-}\right), 0, w^{-}\left(v_{*}^{-}\right), r^{-}\left(v_{*}^{-}\right)\right)\right. \\
& \left.\left.=\gamma_{\text {het }}^{-}\left(\xi_{\text {het }}^{-} ; v_{*}^{-}\right)\right): v_{*}^{-} \in\left(-1, S_{v}^{-}\right)\right\},
\end{aligned}
$$

see Fig. 8, where one point on $Z^{-}$is illustrated, since $v_{*}^{-}$is fixed in the figure. The curve $Z^{-}$ is by construction exponentially close to $\mathcal{M}_{\varepsilon}^{+}$, and its projection on $\mathcal{M}_{\varepsilon}^{+}$is given by

$Z_{\text {slow }}^{-}=\left\{\left(1+\varepsilon u_{1}^{+}\left(v^{-}, 0, w^{-}, r^{-}\right), p_{1}^{+}\left(v^{-}, 0, w^{-}, r^{-}\right), v^{-}, 0, w^{-}, r^{-}\right): v_{*}^{-} \in\left(-1, S_{v}^{-}\right)\right\}$, see (2.8).

We perform the same construction in backwards (spatial) time and define the one-parameter family of orbits $\gamma_{\text {het }}^{+}\left(\xi ; v_{*}^{+}\right) \in W^{s}\left(P_{\varepsilon}^{-}\right) \cap W^{u}\left(\mathcal{M}_{\varepsilon}^{+}\right)$by (3.7), the one-dimensional curve $Z^{+}=\left\{\left(u^{+}\left(v_{*}^{+}\right), p^{+}\left(v_{*}^{+}\right), v^{+}\left(v_{*}^{+}\right), 0, w^{+}\left(v_{*}^{+}\right), r^{+}\left(v_{*}^{+}\right)\right)\right\} \subset\{q=0\}$, and its projection $Z_{\text {slow }}^{+}$on $\mathcal{M}_{\varepsilon}^{+}$. The $(w, r)$-components of $Z_{\text {slow }}^{ \pm}$define two curves, that typically intersect, i.e., the condition $\left(w^{-}\left(v_{*}^{-}\right), r^{-}\left(v_{*}^{-}\right)\right)=\left(w^{+}\left(v_{*}^{+}\right), r^{+}\left(v_{*}^{+}\right)\right)$determines for each given $c$ a discrete number of critical values $\left(v_{*, *}^{-}(c), v_{*, *}^{+}(c)\right)$. However, for general $c$, the one-dimensional curves $Z_{\text {slow }}^{-}$and $Z_{\text {slow }}^{+}$do not intersect within the three-dimensional manifold $\mathcal{M}_{\varepsilon}^{+}$, i.e., $v^{-}\left(v_{*, *}^{-}(c)\right) \neq v^{+}\left(v_{*, *}^{+}(c)\right)$ in general. Nevertheless, the combined condition,

$$
\left(v^{-}\left(v_{*}^{-}(c)\right), w^{-}\left(v_{*}^{-}(c)\right), r^{-}\left(v_{*}^{-}(c)\right)\right)=\left(v^{+}\left(v_{*}^{+}(c)\right), w^{+}\left(v_{*}^{+}(c)\right), r^{+}\left(v_{*}^{+}(c)\right)\right),
$$

in principle determines discrete critical values $c_{j}$ of $c$ for which $Z_{\text {slow }}^{-}$and $Z_{\text {slow }}^{+}$intersect (transversely) in $\mathcal{M}_{\varepsilon}^{+}$. It is a matter of straightforward calculations to show that (3.17) is equivalent to (3.13).

The present construction is computationally more cumbersome than that of Sect. 3.1, but its character is more geometrical and it can thus be more easily extended into a validity proof. 
To do so, we define (for any $c$ ) the special heteroclinic orbits $\gamma_{*, *}^{-}(\xi ; c)=\gamma_{\text {het }}^{-}\left(\xi ; v_{*, *}^{-}\right) \subset$ $W^{u}\left(P_{\varepsilon}^{-}\right) \cap W^{s}\left(\mathcal{M}_{\varepsilon}^{+}\right)$and $\gamma_{*, *}^{+}(\xi ; c)=\gamma_{\text {het }}^{+}\left(\xi ; v_{*, *}^{+}\right) \subset W^{s}\left(P_{\varepsilon}^{-}\right) \cap W^{u}\left(\mathcal{M}_{\varepsilon}^{+}\right)$. The tube $\mathcal{T}_{*, *}^{-}(c) \subset W^{u}\left(P_{\varepsilon}^{-}\right)$is spanned by those orbits $\gamma_{P}^{-}\left(\xi ; v_{*}^{-}, w_{*}^{-}\right) \subset W^{u}\left(P_{\varepsilon}^{-}\right)$that are exponentially close to $\gamma_{*, *}^{-}(\xi ; c)$ for $\xi<\frac{1}{2}\left(-\xi_{*}+\xi_{\text {het }}^{-}\left(v_{*, *}^{-}\right)\right)$. Likewise, the tube $\mathcal{T}_{*, *}^{+}(c) \subset W^{s}\left(P_{\varepsilon}^{-}\right)$is spanned by those orbits $\gamma_{P}^{+}\left(\xi ; v_{*}^{-}, w_{*}^{-}\right) \subset W^{s}\left(P_{\varepsilon}^{-}\right)$that are exponentially close to $\gamma_{*, *}^{+}(\xi ; c)$ for $\xi>\frac{1}{2}\left(\xi_{*}+\xi_{\text {het }}^{+}\left(v_{*, *}^{+}\right)\right)$. In forwards 'time', $\mathcal{T}_{*, *}^{-}(c)$ is stretched along $W^{u}\left(\mathcal{M}_{\varepsilon}^{+}\right)$, while $\mathcal{T}_{*, *}^{+}(c)$ is stretched along $W^{s}\left(\mathcal{M}_{\varepsilon}^{+}\right)$in backwards 'time'. By construction, the (stretched) tubes intersect the five-dimensional hyperplane $\{q=0\}$ in two-dimensional manifolds, $Z_{\mathcal{T}}^{ \pm}(c)$ (by definition).

The theorem is proved if it can be established that there are non-zero values of $c$ for which $Z_{\mathcal{T}}^{-}(c) \cap Z_{\mathcal{T}}^{+}(c) \neq \varnothing$, since each point in this intersection determines a point in $W^{u}\left(P_{\varepsilon}^{-}\right) \cap W^{s}\left(P_{\varepsilon}^{-}\right) \cap\{q=0\}$.

To show this, we extend $\{q=0\}$ to a six-dimensional space, denoted by $\{\{q=0\}, c\}$, by adding $c$ as an independent variable. This space contains the extended manifolds $\left\{Z_{\mathcal{T}}^{-}(c), c\right\}$ and $\left\{Z_{\mathcal{T}}^{+}(c), c\right\}$ as three-dimensional subsets. Since $\gamma_{*, *}^{-}(\xi ; c)$ and $\gamma_{*, *}^{+}(\xi ; c)$ are exponentially close to $\mathcal{M}_{\varepsilon}^{+}$as they intersect $\{q=0\}$, and since the projections $Z_{\text {slow }}^{-}$and $Z_{\text {slow }}^{+}$intersect by construction near $c=c_{j}$ determined by (3.13), it follows that $\left\{Z_{\mathcal{T}}^{-}(c), c\right\}$ and $\left\{Z_{\mathcal{T}}^{+}(c), c\right\}$ are exponentially close for $c$ near $c_{j}$. As in the proof of Theorem 2.1, it now follows from the fact that $\mathcal{T}_{*, *}^{-}(c)$ is stretched along $W^{u}\left(\mathcal{M}_{\varepsilon}^{+}\right)$and $\mathcal{T}_{*, *}^{+}(c)$ along $W^{s}\left(\mathcal{M}_{\varepsilon}^{+}\right)$, that-in the six-dimensional space $\{\{q=0\}, c\}$ - the three-dimensional manifolds $\left\{Z_{\mathcal{T}}^{-}(c), c\right\}$ and $\left\{Z_{\mathcal{T}}^{+}(c), c\right\}$ must intersect transversely in discrete points that have $c$-coordinates $c_{j}^{*}(\varepsilon)$, which are to leading order determined by (3.13) or (3.17). Hence, $Z_{\mathcal{\mathcal { T }}}^{-}(c) \cap Z_{\mathcal{T}}^{+}(c)=\gamma_{t r, j}^{-}(\xi) \cap\{q=0\} \neq \emptyset$ at $c_{j}^{*}(\varepsilon)=c_{j}+\mathcal{O}(\varepsilon)$.

\section{Bifurcation from Stationary to Travelling Pulse Solutions}

\subsection{Leading Order Analysis}

To investigate the nature of the bifurcation from stationary one-pulse solutions to travelling one-pulse solutions, we start by considering the travelling pulse just after 'creation', that is, we set

$$
c=\delta,
$$

with $0<\varepsilon \ll \delta \ll 1$ (so $c$ is no longer an unknown anymore). We expand the three unknowns, $\hat{\tau}=\hat{\tau}_{*, 0}+\mathcal{O}(\delta), \hat{\theta}=\hat{\theta}_{*, 0}+\mathcal{O}(\delta), \xi_{*}=\xi_{*, 0}+\delta \xi_{*, 1}+\mathcal{O}\left(\delta^{2}\right)$. Notice that $\hat{\tau}_{*, 0}$ and $\hat{\theta}_{*, 0}$ determine the bifurcation values of $\hat{\tau}$ and $\hat{\theta}$ at which the bifurcation occurs, since the speed of the bifurcating travelling pulse reduces to zero at $\hat{\tau}=\hat{\tau}_{*, 0}$ and $\hat{\theta}=\hat{\theta}_{*, 0}$. Since the bifurcation is co-dimension one we expect to find a relation between $\hat{\tau}_{*, 0}$ and $\hat{\theta}_{*, 0}$.

The eigenvalues (3.8) and (3.12) become

$$
\begin{aligned}
& \lambda_{v}^{ \pm}= \pm 1-\frac{1}{2} \hat{\tau}_{*, 0} \delta+\mathcal{O}\left(\delta^{2}\right), \quad \lambda_{w}^{ \pm}= \pm \frac{1}{D}-\frac{1}{2} \frac{\hat{\theta}_{*, 0}}{D^{2}} \delta+\mathcal{O}\left(\delta^{2}\right), \\
& s_{v}^{ \pm}=-1 \pm \frac{1}{2} \hat{\tau}_{*, 0} \delta+\mathcal{O}\left(\delta^{2}\right), \quad s_{w}^{ \pm}=-1 \pm \frac{1}{2} \frac{\hat{\theta}_{*, 0}}{D} \delta+\mathcal{O}\left(\delta^{2}\right) .
\end{aligned}
$$




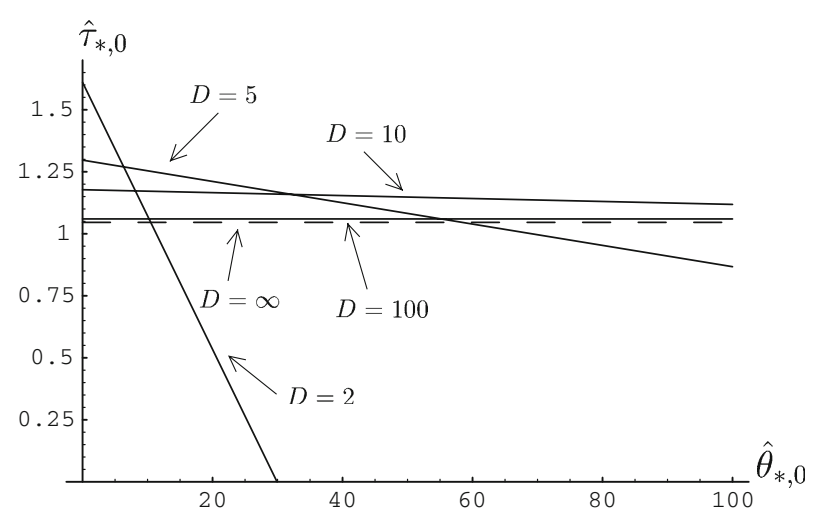

Fig. 9 For $(\alpha, \beta, \gamma, \varepsilon)=(3,1,2,0.01)$, the bifurcation point $\hat{\tau}_{*, 0}\left(\hat{\theta}_{*, 0}\right)$ is plotted for $D=2,5,10,100$. The value of the jump mid-point $\xi_{*, 0}$ is, respectively, 40.547, 47.018, 50.356, 54.393 and is computed through (4.2). When $D=\infty$, we have $\xi_{*, 0}=54.931$ and $\hat{\tau}_{*, 0}\left(\hat{\theta}_{*, 0}\right)=\hat{\tau}_{*, 0}=1.0460$. This is the dotted line in the figure

We also expand the four equalities in (3.11), using $A_{0}:=e^{-\varepsilon \xi_{*, 0}}$,

$$
\begin{aligned}
& v_{*}^{ \pm}=-A_{0}^{2} \mp \hat{\tau}_{*, 0} \delta\left(\frac{1}{2}-\frac{1}{2} A_{0}^{2}+A_{0}^{2} \log A_{0}\right)+2 \varepsilon \xi_{*, 1} A_{0}^{2} \delta+\mathcal{O}\left(\delta^{2}\right), \\
& w_{*}^{ \pm}=-A_{0}^{\frac{2}{D}} \mp \frac{\hat{\theta}_{*, 0}}{D} \delta\left(\frac{1}{2}-\frac{1}{2} A_{0}^{\frac{2}{D}}+\frac{1}{D} A_{0}^{\frac{2}{D}} \log A_{0}\right)+2 \frac{\varepsilon}{D} \xi_{*, 1} A_{0}^{\frac{2}{D}} \delta+\mathcal{O}\left(\delta^{2}\right) .
\end{aligned}
$$

Next, we substitute the above expansions into the jump condition (3.13), and we recall that $c=\delta$, to obtain

$$
\left\{\begin{aligned}
\gamma & =\alpha A_{0}^{2}+\beta A_{0}^{\frac{2}{D}} \quad \text { (twice), } \\
\frac{1}{3} \sqrt{2} & =\alpha \hat{\tau}_{*, 0}\left(\frac{1}{2}-\frac{1}{2} A_{0}^{2}+A_{0}^{2} \log A_{0}\right)+\beta \frac{\hat{\theta}_{*, 0}}{D}\left(\frac{1}{2}-\frac{1}{2} A_{0}^{\frac{2}{D}}+\frac{1}{D} A_{0}^{\frac{2}{D}} \log A_{0}\right), \\
0 & =4 \varepsilon \xi_{*, 1}\left(\alpha A_{0}^{2}+\frac{\beta}{D} A_{0}^{\frac{2}{D}}\right),
\end{aligned}\right.
$$

where we equated coefficients on $\mathcal{O}(1)$ and $\mathcal{O}(\delta)$ terms, respectively, and added and subtracted the two $\mathcal{O}(\delta)$ equations. Note that the equation for $A_{0}$ is identical to that of the stationary one-pulse orbit (2.22): near the bifurcation the width of the travelling pulse is to leading order equal to that of the stationary pulse. Equation 4.2 determine the three unknowns $A_{0}$ (which gives $\xi_{*, 0}$ ), $\hat{\tau}_{*, 0}$ as function of $\hat{\theta}_{*, 0}$, and $\xi_{*, 1}=0$. The solution $\hat{\tau}_{*, 0}$ as function of $\hat{\theta}_{*, 0}$, is plotted in Fig. 9 for several values of $D$.

Remark 4.1 We briefly consider the case of $D$ large, i.e., $D=\mathcal{O}\left(\frac{1}{\delta}\right)$. It immediately follows from (4.2) that $\xi_{*, 0}=-\frac{1}{2} \frac{1}{\varepsilon} \log \left(\frac{\gamma-\beta}{\alpha}\right)$. (Here, we also have to assume that $\gamma>\beta, \alpha>0$ or that $\gamma<\beta, \alpha<0)$. Moreover,

$$
\hat{\tau}_{*, 0}(\hat{\theta})=\frac{2}{3} \sqrt{2}\left(\alpha-(\gamma-\beta)+(\gamma-\beta) \log \left(\frac{\gamma-\beta}{\alpha}\right)\right)^{-1}+\mathcal{O}(\delta) .
$$

This $\hat{\tau}_{*, 0}$ is analogous to the $\left(\hat{\tau}_{2}\right)_{*, 0}$ we find in the analysis for travelling pulses of the reduced two-component system (6.1)—see Sect. 6. 


\subsection{Subcriticality and Supercriticality of the Bifurcation}

To determine the nature (supercritical versus subcritical) of the bifurcation, see Fig. 11, and also for the stability analysis [23], we actually need the correction terms up to and including third order in $\delta$ in the above calculations. To keep the calculations within reasonable limits, we set the bifurcation parameter $\theta$ equal to one, such that in the above analysis the $w$-component is symmetric and has no higher order corrections, i.e., $\hat{\theta}=0$ in (3.8), etc. Note that $\theta$ has also been set to $\theta=1$ in [17,24,25]. Moreover, most of the numerical results presented in $[2,15,18,22]$ are for $\theta=1$. We also assume that $\alpha A_{0}^{2}+\frac{\beta}{D} A_{0}^{2 / D}>0$, which implies that the stationary one-pulse limit is not near a saddle-node bifurcation and that it is stable [23].

Lemma 4.1 Let $(\alpha, \beta, \gamma, D, \tau, \theta, \varepsilon)$ be such that $\tau=\mathcal{O}\left(\frac{1}{\varepsilon^{2}}\right), \theta=1, \alpha>0$, (2.22) holds, and $\alpha A_{0}^{2}+\frac{\beta}{D} A_{0}^{2 / D}>0$, where $A_{0}=e^{-\varepsilon \xi_{*, 0}}$ and $0<\varepsilon \ll 1$. For $c=\delta$, with $\varepsilon \ll \delta \ll 1, a$ travelling pulse exists for $\tau=\frac{1}{\varepsilon^{2}}\left(\hat{\tau}_{*, 0}+\delta^{2} \hat{\tau}_{*, 2}+\mathcal{O}\left(\delta^{3}\right)\right)$, with

$$
\begin{aligned}
\hat{\tau}_{*, 0}= & \frac{2}{3} \sqrt{2} \frac{1}{\alpha\left(1-A_{0}^{2}+A_{0}^{2} \log A_{0}^{2}\right)}>0, \\
\hat{\tau}_{*, 2}= & \frac{3}{32} \sqrt{2} \alpha\left(\hat{\tau}_{*, 0}\right)^{4}\left[1-A_{0}^{2}+A_{0}^{2} \log A_{0}^{2}-\frac{1}{3} A_{0}^{2} \log ^{3} A_{0}^{2}\right. \\
& +\frac{\alpha A_{0}^{4} \log ^{2} A_{0}^{2}\left(\log A_{0}^{2}-1\right)}{\left.\alpha A_{0}^{2}+\frac{\beta}{D} A_{0}^{2 / D}\right] .}
\end{aligned}
$$

Note that the sign of $\hat{\tau}_{*, 2}$ determines the nature of the bifurcation: a negative $\hat{\tau}_{*, 2}$ yields a subcritical bifurcation, while a positive $\hat{\tau}_{*, 2}$ yields a supercritical bifurcation. For given system parameters, we can evaluate (4.3) to determine the sign of $\hat{\tau}_{*, 2}$. Moreover, we observe that it is possible for the same $(\alpha, \beta, D)$ for $\hat{\tau}_{*, 2}$ to take on positive, as well as negative, values, depending on $\gamma$ (via $A_{0}$ ), as is illustrated in Fig. 10.

Proof The proof consists of an elaborate-but straightforward-asymptotic analysis of the jump conditions (3.13). Plugging in $v_{*}^{ \pm}, w_{*}^{ \pm}$with $\theta=1$ yields, to leading order in $\varepsilon$,

$$
\alpha\left(s_{v}^{ \pm}\left(e^{ \pm 2 \varepsilon \lambda_{v}^{\mp} \xi_{*}}-1\right)-1\right)-\beta e^{-2 \frac{\varepsilon}{D} \xi_{*}}+\gamma=\mp \frac{1}{3} \sqrt{2} c .
$$
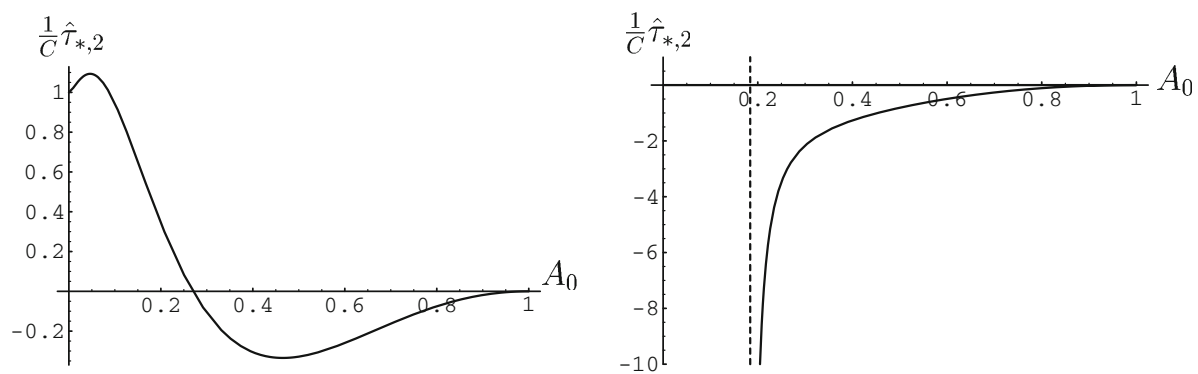

Fig. 10 Left frame: $(\alpha, \beta, D)=(3,1,5)$. Right frame: $(\alpha, \beta, D)=(3,-1,5)$. Note that we did not plot $\hat{\tau}_{*, 2}$ but a 'scaled' version $\hat{\tau}_{*, 2} / C$. To be more precise, $C=\frac{3}{32} \sqrt{2} \alpha\left(\hat{\tau}_{*, 0}\right)^{4}$, and the scaling therefore depends on $A_{0}$. However, $C>0$ for $A_{0} \in(0,1)$. Thus, the scaling does not change the sign of $\hat{\tau}_{*, 2}$. Moreover, note that the vertical asymptote (for $\beta<0$ ) is exactly where $\alpha A_{0}^{2}+\frac{\beta}{D} A_{0}^{\frac{2}{D}}=0\left(A_{0}=A_{c}\right.$, see (2.25)). The last free parameter, $\gamma$, actually determines the value of $A_{0}$ via (4.7). Thus for $(\alpha, \beta, D)=(3,1,5)$ it is possible to have a negative, as well as a positive $\hat{\tau}_{*, 2}$ 
After expanding the two unknown variables $\hat{\tau}$ and $\xi_{*}$,

$$
\hat{\tau}=\hat{\tau}_{*, 0}+\delta \hat{\tau}_{*, 1}+\delta^{2} \hat{\tau}_{*, 2}+\delta^{3} \hat{\tau}_{*, 3}+\mathcal{O}\left(\delta^{4}\right), \quad \xi_{*}=\xi_{*, 0}+\delta \xi_{*, 1}+\delta^{2} \xi_{*, 2}+\delta^{3} \xi_{*, 3}+\mathcal{O}\left(\delta^{4}\right),
$$

we obtain the leading order approximations of (3.8) and (3.12),

$$
\begin{aligned}
& \lambda_{v}^{ \pm}= \pm 1-\frac{1}{2} \hat{\tau}_{*, 0} \delta+\left( \pm \frac{1}{8} \hat{\tau}_{*, 0}^{2}-\frac{1}{2} \hat{\tau}_{*, 1}\right) \delta^{2}+\left( \pm \frac{1}{4} \hat{\tau}_{*, 0} \hat{\tau}_{*, 1}-\frac{1}{2} \hat{\tau}_{*, 2}\right) \delta^{3}+\mathcal{O}\left(\delta^{4}\right), \\
& s_{v}^{ \pm}=-1 \pm \frac{1}{2} \hat{\tau}_{*, 0} \delta \pm \frac{1}{2} \hat{\tau}_{*, 1} \delta^{2} \mp\left(\frac{1}{16} \hat{\tau}_{*, 0}^{3}-\frac{1}{2} \hat{\tau}_{*, 2}\right) \delta^{3}+\mathcal{O}\left(\delta^{4}\right) .
\end{aligned}
$$

With these expressions we deduce

$$
\begin{aligned}
e^{ \pm 2 \varepsilon \lambda_{v}^{\mp} \xi_{*}}= & e^{-2 \varepsilon \xi_{*, 0}}+e^{-2 \varepsilon \xi_{*, 0}}\left(\mp \varepsilon \hat{\tau}_{0} \xi_{*, 0}-2 \varepsilon \xi_{*, 1}\right) \delta+e^{-2 \varepsilon \xi_{*, 0}}\left[-\frac{1}{4} \varepsilon\left(\hat{\tau}_{*, 0}\right)^{2} \xi_{*, 0} \mp \varepsilon \hat{\tau}_{*, 1} \xi_{*, 0}\right. \\
& \left.\mp \varepsilon \hat{\tau}_{*, 0} \xi_{*, 1} \pm 2 \varepsilon^{2} \hat{\tau}_{*, 0} \xi_{*, 0} \xi_{*, 1}+\frac{1}{2} \varepsilon^{2}\left(\hat{\tau}_{*, 0}\right)^{2}\left(\xi_{*, 0}\right)^{2}+2 \varepsilon^{2}\left(\xi_{*, 1}\right)^{2}-2 \varepsilon \xi_{*, 2}\right] \delta^{2} \\
& +e^{-2 \varepsilon \xi_{*, 0}}\left[-\frac{1}{2} \varepsilon \hat{\tau}_{*, 0} \hat{\tau}_{*, 1} \xi_{*, 0} \mp \varepsilon \hat{\tau}_{2} \xi_{*, 0} \pm \frac{1}{4} \varepsilon^{2}\left(\hat{\tau}_{*, 0}\right)^{3}\left(\xi_{*, 0}\right)^{2}+\varepsilon^{2} \hat{\tau}_{*, 0} \hat{\tau}_{*, 1}\left(\xi_{*, 0}\right)^{2}\right. \\
& \mp \frac{1}{6} \varepsilon^{3}\left(\hat{\tau}_{*, 0}\right)^{3}\left(\xi_{*, 0}\right)^{3}-\frac{1}{4} \varepsilon\left(\hat{\tau}_{*, 0}\right)^{2} \xi_{*, 1} \mp \varepsilon \hat{\tau}_{*, 1} \xi_{*, 1}+\frac{3}{2} \varepsilon^{2}\left(\hat{\tau}_{*, 0}\right)^{2} \xi_{*, 0} \xi_{*, 1} \\
& \pm 2 \varepsilon^{2} \hat{\tau}_{*, 1} \xi_{*, 0} \xi_{*, 1}-\varepsilon^{3}\left(\hat{\tau}_{*, 0}\right)^{2}\left(\xi_{*, 0}\right)^{2} \xi_{*, 1} \pm 2 \varepsilon^{2} \hat{\tau}_{*, 0}\left(\xi_{*, 1}\right)^{2} \mp 2 \varepsilon^{3} \hat{\tau}_{*, 0} \xi_{*, 0}\left(\xi_{*, 1}\right)^{2} \\
& \left.-\frac{4}{3} \varepsilon^{3}\left(\xi_{*, 1}\right)^{3} \mp \varepsilon \hat{\tau}_{*, 0} \xi_{*, 2} \pm 2 \varepsilon^{2} \hat{\tau}_{*, 0} \xi_{*, 0} \xi_{*, 2}+4 \varepsilon^{2} \xi_{*, 1} \xi_{*, 2}-2 \varepsilon \xi_{*, 3}\right] \delta^{3}+\mathcal{O}\left(\delta^{4}\right),
\end{aligned}
$$

and

$$
\begin{aligned}
e^{-2 \frac{\varepsilon}{D} \xi_{*}}= & e^{-2 \frac{\varepsilon}{D} \xi_{*, 0}}-\frac{2}{D} \varepsilon \xi_{*, 1} e^{-2 \frac{\varepsilon}{D} \xi_{*, 0}} \delta+e^{-2 \frac{\varepsilon}{D} \xi_{*, 0}}\left[\frac{2}{D^{2}} \varepsilon^{2}\left(\xi_{*, 1}\right)^{2}-\frac{2}{D} \varepsilon \xi_{*, 2}\right] \delta^{2} \\
& +e^{-2 \frac{\varepsilon}{D} \xi_{*, 0}}\left[-\frac{4}{3 D^{3}} \varepsilon^{3}\left(\xi_{*, 1}\right)^{3}+\frac{4}{D^{2}} \varepsilon^{2} \xi_{*, 1} \xi_{*, 2}-\frac{2}{D} \varepsilon \xi_{*, 3}\right] \delta^{3}+\mathcal{O}\left(\delta^{4}\right) .
\end{aligned}
$$

(Recall that $\varepsilon \xi_{*, j}=\mathcal{O}(1)$.)

Combining (4.4), (4.5), and (4.6), we find to leading order (twice)

$$
\alpha A_{0}^{2}+\beta A_{0}^{\frac{2}{D}}=\gamma
$$

which agrees with the first equation in (4.2).

The $\mathcal{O}(\delta)$ corrections read

$$
\pm \frac{1}{2} \alpha \hat{\tau}_{*, 0}\left(1-A_{0}^{2}+A_{0}^{2} \log A_{0}^{2}\right)+2 \varepsilon \xi_{*, 1}\left(\alpha A_{0}^{2}+\frac{\beta}{D} A_{0}^{\frac{2}{D}}\right)= \pm \frac{1}{3} \sqrt{2} .
$$

By adding and subtracting the above two equations, we obtain

$$
\xi_{*, 1}=0, \quad \hat{\tau}_{*, 0}=\frac{2}{3} \sqrt{2} \frac{1}{\alpha\left(1-A_{0}^{2}+A_{0}^{2} \log A_{0}^{2}\right)},
$$

which agrees with (4.2), since $\hat{\theta}_{*, 0}=0$. Note that the function $1-A_{0}^{2}+A_{0}^{2} \log A_{0}^{2}$ is positive for all $A_{0} \in(0,1)$ - it decreases monotonically from one to zero as $A_{0}$ increases from zero to one. Since $\alpha>0$ it follows that $\hat{\tau}_{*, 0}>0$. 
At $\mathcal{O}\left(\delta^{2}\right)$, we find

$$
\begin{aligned}
0= & \pm \frac{1}{2} \alpha \hat{\tau}_{*, 1}\left(A_{0}^{2}-1\right)-\alpha A_{0}^{2}\left[-\frac{1}{4} \varepsilon\left(\hat{\tau}_{*, 0}\right)^{2} \xi_{*, 0} \mp \varepsilon \hat{\tau}_{1} \xi_{*, 0}+\frac{1}{2} \varepsilon^{2}\left(\hat{\tau}_{*, 0}\right)^{2}\left(\xi_{*, 0}\right)^{2}-2 \varepsilon \xi_{*, 2}\right] \\
& -\frac{1}{2} \varepsilon \alpha\left(\hat{\tau}_{*, 0}\right)^{2} \xi_{*, 0} A_{0}^{2}+2 \frac{\beta}{D} \varepsilon \xi_{*, 2} A_{0}^{\frac{2}{D}},
\end{aligned}
$$

(since $\xi_{*, 1}=0$ ). Subtracting the two equalities implies

$$
\alpha \hat{\tau}_{*, 1}\left(1-A_{0}^{2}+A_{0}^{2} \log A_{0}^{2}\right)=0 \Rightarrow \hat{\tau}_{*, 1}=0
$$

Adding both terms yields

$$
\xi_{*, 2}=\frac{1}{16} \frac{1}{\varepsilon} \frac{\alpha A_{0}^{2}\left(\hat{\tau}_{*, 0}\right)^{2} \log A_{0}^{2}\left(\log A_{0}^{2}-1\right)}{\alpha A_{0}^{2}+\frac{\beta}{D} A_{0}^{2 / D}} .
$$

We note that $\log A_{0}^{2}-1<\log A_{0}^{2}<0$ and $\alpha A_{0}^{2}+\frac{\beta}{D} A_{0}^{2 / D}>0$, therefore, $\operatorname{sgn}\left(\xi_{*, 2}\right)=$ $\operatorname{sgn}(\alpha)=+1$. Thus, the width of the pulse $\left(2 \xi_{*}\right)$ is larger than the leading order width $\left(2 \xi_{*, 0}\right)$, i.e., the width of the travelling pulse is larger than the width of the standing pulse.

The $\mathcal{O}\left(\delta^{3}\right)$-term is given by

$$
\begin{aligned}
0= & \pm \alpha\left(\frac{1}{16}\left(\hat{\tau}_{*, 0}\right)^{3}-\frac{1}{2} \hat{\tau}_{*, 2}\right)\left(A_{0}^{2}-1\right) \mp \frac{1}{2} \alpha \hat{\tau}_{*, 0} A_{0}^{2}\left[-\frac{1}{4} \varepsilon\left(\hat{\tau}_{*, 0}\right)^{2} \xi_{*, 0}\right. \\
& \left.+\frac{1}{2} \varepsilon^{2}\left(\hat{\tau}_{*, 0}\right)^{2}\left(\xi_{*, 0}\right)^{2}-2 \varepsilon \xi_{*, 2}\right] \\
& \mp \alpha A_{0}^{2}\left[\varepsilon \hat{\tau}_{*, 2} \xi_{*, 0}-\frac{1}{4} \varepsilon^{2}\left(\hat{\tau}_{*, 0}\right)^{3}\left(\xi_{*, 0}\right)^{2}+\frac{1}{6} \varepsilon^{3}\left(\hat{\tau}_{*, 0}\right)^{3}\left(\xi_{*, 0}\right)^{3}\right. \\
& \left.+\varepsilon \hat{\tau}_{*, 0} \xi_{*, 2}-2 \varepsilon^{2} \hat{\tau}_{*, 0} \xi_{*, 0} \xi_{*, 2} \mp 2 \varepsilon \xi_{*, 3}\right] \\
& +2 \frac{\beta}{D} \varepsilon \xi_{*, 3} A_{0}^{\frac{2}{D}} .
\end{aligned}
$$

Adding both terms implies $\xi_{*, 3}=0$, subtracting yields,

$$
\begin{aligned}
& \alpha\left(\frac{1}{16}\left(\hat{\tau}_{*, 0}\right)^{3}-\frac{1}{2} \hat{\tau}_{*, 2}\right)\left(A_{0}^{2}-1\right)-\frac{1}{2} \alpha \hat{\tau}_{*, 0} A_{0}^{2}\left[-\frac{1}{4} \varepsilon\left(\hat{\tau}_{*, 0}\right)^{2} \xi_{*, 0}+\frac{1}{2} \varepsilon^{2}\left(\hat{\tau}_{*, 0}\right)^{2}\left(\xi_{*, 0}\right)^{2}\right. \\
& \left.-2 \varepsilon \xi_{*, 2}\right]-\alpha A_{0}^{2}\left[\varepsilon \hat{\tau}_{*, 2} \xi_{*, 0}-\frac{1}{4} \varepsilon^{2}\left(\hat{\tau}_{*, 0}\right)^{3}\left(\xi_{*, 0}\right)^{2}+\frac{1}{6} \varepsilon^{3}\left(\hat{\tau}_{*, 0}\right)^{3}\left(\xi_{*, 0}\right)^{3}+\varepsilon \hat{\tau}_{*, 0} \xi_{*, 2}\right. \\
& \left.-2 \varepsilon^{2} \hat{\tau}_{*, 0} \xi_{*, 0} \xi_{*, 2}\right]=0
\end{aligned}
$$

which can be rewritten as

$$
\begin{aligned}
0= & -\alpha A_{0}^{2} \hat{\tau}_{*, 0} \varepsilon \xi_{*, 2} \log A_{0}^{2}+\frac{1}{48} \alpha A_{0}^{2}\left(\hat{\tau}_{*, 0}\right)^{3} \log ^{3} A_{0}^{2}-\frac{1}{16} \alpha\left(\hat{\tau}_{*, 0}\right)^{3}\left(1-A_{0}^{2}+A_{0}^{2} \log A_{0}^{2}\right) \\
& +\frac{1}{2} \alpha \hat{\tau}_{*, 2}\left(1-A_{0}^{2}+A_{0}^{2} \log A_{0}^{2}\right) .
\end{aligned}
$$


Then, using the expression for $\hat{\tau}_{*, 0}$ and $\xi_{*, 2}$, we obtain

$$
\hat{\tau}_{*, 2}=\frac{1}{8}\left(\hat{\tau}_{*, 0}\right)^{3}-\frac{1}{32} \sqrt{2} \alpha A_{0}^{2}\left(\hat{\tau}_{*, 0}\right)^{4} \log ^{3} A_{0}^{2}+\frac{3}{32} \sqrt{2} \frac{\alpha^{2} A_{0}^{4}\left(\hat{\tau}_{*, 0}\right)^{4} \log ^{2} A_{0}^{2}\left(\log A_{0}^{2}-1\right)}{\alpha A_{0}^{2}+\frac{\beta}{D} A_{0}^{2 / D}},
$$

which can be rewritten as in (4.3).

For $D$ large, we can analytically determine the sign of $\hat{\tau}_{*, 2}$ in (4.3), as we now show.

Corollary 4.2 Let $(\alpha, \beta, \gamma, D, \tau, \theta, \varepsilon)$ and $A_{0}$ be as in Lemma 4.1 and assume that $D=\frac{1}{\delta}$ with $0<\varepsilon \ll \delta \ll 1$. Define $A_{0}^{Z} \in(0,1)$ as the (unique) solution of

$$
1-A_{0}^{2}+A_{0}^{2} \log A_{0}^{2}+\frac{2}{3} A_{0}^{2} \log ^{3} A_{0}^{2}-A_{0}^{2} \log ^{2} A_{0}^{2}=0,
$$

$\left(A_{0}^{Z}=0.11063 \ldots\right)$. Then, $\hat{\tau}_{*, 2}>0$ for parameter combinations such that $0<A_{0}<$ $A_{0}^{Z}+\mathcal{O}(\delta)$ and $\hat{\tau}_{*, 2}<0$ for $1>A_{0}>A_{0}^{Z}+\mathcal{O}(\delta)$.

Proof It follows from (4.3) that, to leading order in $\delta$,

$$
\begin{aligned}
\left.\hat{\tau}_{*, 2}\right|_{D=\mathcal{O}\left(\delta^{-1}\right)=} & \frac{3}{32} \sqrt{2} \alpha\left(\hat{\tau}_{*, 0}\right)^{4}\left[1-A_{0}^{2}+A_{0}^{2} \log A_{0}^{2}\right. \\
& \left.-\frac{1}{3} A_{0}^{2} \log ^{3} A_{0}^{2}+A_{0}^{2} \log ^{2} A_{0}^{2}\left(\log A_{0}^{2}-1\right)\right] \\
= & \frac{3}{32} \sqrt{2} \alpha\left(\hat{\tau}_{*, 0}\right)^{4}\left[1-A_{0}^{2}+A_{0}^{2} \log A_{0}^{2}+\frac{2}{3} A_{0}^{2} \log ^{3} A_{0}^{2}\right. \\
& \left.-A_{0}^{2} \log ^{2} A_{0}^{2}\right]=: C \hat{\tau}_{*, 2}^{\prime},
\end{aligned}
$$

with $C=\frac{3}{32} \sqrt{2} \alpha\left(\hat{\tau}_{*, 0}\right)^{4}>0$ and $\hat{\tau}_{*, 2}^{\prime}=1-A_{0}^{2}+A_{0}^{2} \log A_{0}^{2}+\frac{2}{3} A_{0}^{2} \log ^{3} A_{0}^{2}-A_{0}^{2} \log ^{2} A_{0}^{2}$. Thus $\operatorname{sgn}\left(\hat{\tau}_{*, 2}\right)=\operatorname{sgn}\left(\hat{\tau}_{*, 2}^{\prime}\right)$. We notice that $\hat{\tau}_{*, 2}^{\prime}(0)=1$ and $\hat{\tau}_{*, 2}^{\prime}(1)=0$. We now show that $\hat{\tau}_{*, 2}^{\prime}(s)$, with $s:=A_{0}^{2}$, has a negative minimum by differentiating,

$$
\frac{d}{d s} \hat{\tau}_{*, 2}^{\prime}=(\log s)\left(\frac{2}{3} \log ^{2} s+\log s-1\right) .
$$

Thus, with $z:=\log s$ (so that $z \in(-\infty, 0)$ ), we see that $\hat{\tau}_{*, 2}^{\prime}(z)$ has a unique extremum if $\frac{2}{3} z^{2}+z-1=0$, i.e., $z=z^{M}=-\frac{3}{4}-\frac{1}{4} \sqrt{33}$. This implies that $A_{0}^{M}=e^{-\frac{1}{8}(3+\sqrt{33})} \in(0,1)$, so that

$$
\hat{\tau}_{*, 2}^{\prime}\left(A_{0}^{M}\right)=1-e^{-\frac{1}{4}(3+\sqrt{33})}\left(\frac{31}{4}+\frac{5}{4} \sqrt{33}\right)<0 .
$$

Hence, $A_{0}^{M}$ determines a negative minimum of $\hat{\tau}_{*, 2}^{\prime}$, which implies $\hat{\tau}_{*, 2}^{\prime}$ must change sign once for $A=A_{0}^{Z} \in\left(0, A_{0}^{M}\right)$, where $A_{0}^{Z}$ is determined by (4.9).

An additional consequence of Lemma 4.1, that holds for more general values of $D$, is

Corollary 4.3 Let $(\alpha, \beta, \gamma, D, \tau, \theta, \varepsilon)$ and $A_{0}$ be as in Lemma 4.1. Furthermore, assume that $\beta<0, \alpha D>-\beta, A_{0}>A_{c}>A_{0}^{Z}$ (with $A_{c}, A_{0}^{Z}$ as in (2.25), (4.9), respectively), then the bifurcation is subcritical, i.e., $\hat{\tau}_{*, 2}<0$. 


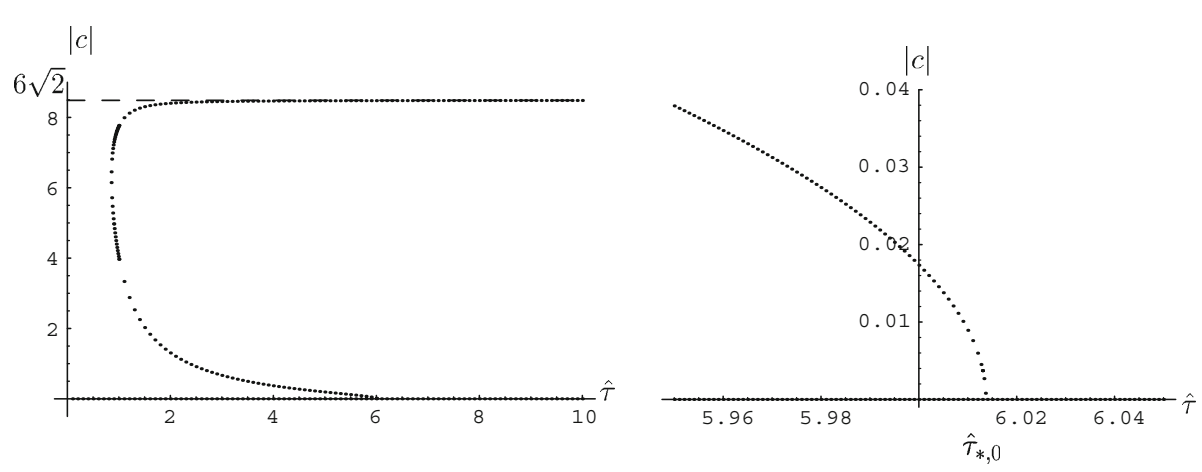

Fig. 11 The solution curve of equation (3.13) in the ( $\hat{\tau}, c)$ plane for the parameter values $(\alpha, \beta, \gamma, D, \theta, \varepsilon)=$ $(5,-3,1,4,1,0.01)$. We have chosen the parameters in such a fashion that they satisfy the conditions in Remark 4.2. In the left frame we observe a subcritical bifurcation at $\hat{\tau}=\hat{\tau}_{*, 0}=6.01363$. Moreover, we observe that as $\hat{\tau}$ goes to infinity the upperbranch, $c_{+}(\hat{\tau})$, goes to the theoretically predicted, leading order value, $\frac{3}{2} \sqrt{2}(\alpha-\gamma)=6 \sqrt{2}$, see (3.15). Finally, from this numerical continuation we observe that the two branches merge at a saddle-node bifurcation at $\hat{\tau}_{S N}^{n u m}=0.84917$ and $c_{S N}^{n u m}=6.3027$. In the right frame, the region near $\hat{\tau}=\hat{\tau}_{*, 0}$ is magnified

Proof Observe that in this case

$$
\frac{\alpha A_{0}^{4} \log ^{2} A_{0}^{2}\left(\log A_{0}^{2}-1\right)}{\alpha A_{0}^{2}+\frac{\beta}{D} A_{0}^{2 / D}}<A_{0}^{2} \log ^{2} A_{0}^{2}\left(\log A_{0}^{2}-1\right)<0 .
$$

Therefore, $\hat{\tau}_{*, 2}\left(A_{0}\right)<C \hat{\tau}_{*, 2}^{\prime}\left(A_{0}\right)$, with $\hat{\tau}_{*, 2}^{\prime}\left(A_{0}\right)$ as defined above, and $C \hat{\tau}_{*, 2}^{\prime}\left(A_{0}\right)$ is negative for $A_{0}>A_{0}^{Z}$.

Remark 4.2 If, in addition to the conditions in Corollary 4.3, it is also assumed that $\alpha>\gamma$, then it follows from our analysis in Sect. 3.2 that there is a travelling pulse with speed $c=\frac{3}{2} \sqrt{2}(\alpha-\gamma)+\mathcal{O}(\delta, \varepsilon)>0$ for $\hat{\tau} \gg 1(3.15)$. This indicates that the curve $c=c(\hat{\tau})$ has a fold structure, i.e., for increasing $\hat{\tau}$ (and all other parameters fixed) there is a saddle-node bifurcation of travelling pulses at $\hat{\tau}=\hat{\tau}_{S N}<\hat{\tau}_{*, 0}$ at which two travelling pulses bifurcate with speeds $c_{ \pm}(\hat{\tau})>0$ and $c_{ \pm}\left(\hat{\tau}_{S N}\right)=c_{S N}>0$; the pulse associated to $c_{-}(\hat{\tau})$ merges with the stationary pulse at $\hat{\tau}=\hat{\tau}_{*, 0}$, while the other pulse exists for all $\hat{\tau}>\hat{\tau}_{S N}$, so that $c_{+}(\hat{\tau}) \rightarrow \frac{3}{2} \sqrt{2}(\alpha-\gamma)$ as $\hat{\tau} \rightarrow \infty$. This can be checked by using a continuation method for the solutions of (3.13), see Fig. 11. Hence, there exist parameter combinations for which two types of travelling pulses coexist with the stationary pulse (for $\hat{\tau}_{S N}<\hat{\tau}<\hat{\tau}_{*, 0}$ ). Both the stationary pulse and the travelling pulse associated to $c_{+}(\hat{\tau})$ may be stable [23].

\section{Stationary Two-Pulse Solutions}

In this section, we establish the existence of localized, symmetric, standing, two-pulse solutions of (1.6). We construct these pulses as homoclinic orbits $\gamma_{2 p, j}^{-}(\xi)$ to the critical point $P_{\varepsilon}^{-}$.

\subsection{The Construction of $\gamma_{2 p, j}^{-}(\xi)$ Homoclinic to $P_{\varepsilon}^{-}$}

We search for stationary pulse-like solutions. Therefore, the PDE (1.7) again reduces to (2.1), and the basic observations (on the fixed points, the reduced limits, the slow manifolds, etc.) are the same as in Sect. 2.1. However, for symmetric standing two-pulse solutions, we have 
to distinguish nine different regions instead of the five regions as we did for the one-pulse solutions-see Sect. 2.2. We again parametrize the two-pulse solutions so that its $u, v, w$ components are at a local extremum at $\xi=0$. However, there are three local extrema, see Fig. 1, and for symmetry considerations we choose to put the zero of the $\xi$-axis at the second location, the one exponentially close to $\mathcal{M}_{\varepsilon}^{-}$. It turns out that $v_{2 p, j}^{-}(0)$ and $w_{2 p, j}^{-}(0)$ are local minima, while $u_{2 p, j}^{-}(0)$ is a local maximum, see Figs. 1 and 12 . We define the four 'jump mid-points' of $\gamma_{2 p, j}^{-}$by $\pm \xi_{*}^{1,2}$ (not to be confused with the $\xi_{*, 1}, \xi_{*, 2}$ of the previous section). Where the last 'back' (i.e., the final jump of $\mathcal{M}_{\varepsilon}^{+}$back to $\mathcal{M}_{\varepsilon}^{-}$) of $\gamma_{2 p, j}^{-}(\xi)$ crosses the $\{u=0\}$-hyperplane at $\xi=\xi_{*}^{1}$, and the last front of $\gamma_{2 p, j}^{-}(\xi)$ crosses the same hyperplane at $\xi=\xi_{*}^{2}$. Note that by construction $0<\xi_{*}^{2}<\xi_{*}^{1}$. The reversibility symmetry implies that $-\xi_{*}^{1}$ is the jump mid-point of the first front and $-\xi_{*}^{2}$ is the jump mid-point of the first back. Thus,

$$
\gamma_{2 p, j}^{-}\left( \pm \xi_{*}^{1}\right)=\left(0, \mp p_{*}^{1}, v_{*}^{1}, \mp q_{*}^{1}, w_{*}^{1}, \mp r_{*}^{1}\right), \gamma_{2 p, j}^{-}\left( \pm \xi_{*}^{2}\right)=\left(0, \pm p_{*}^{2}, v_{*}^{2}, \pm q_{*}^{2}, w_{*}^{2}, \pm r_{*}^{2}\right)
$$

We assume that $\xi_{*}^{1}, \xi_{*}^{2}$, as well as $\xi_{*}^{1}-\xi_{*}^{2}$, are large, i.e., $\xi_{*}^{1,2}$ and $\xi_{*}^{1}-\xi_{*}^{2}$ are $\mathcal{O}\left(\frac{1}{\varepsilon}\right)$. We now define the four fast intervals $I_{f}^{2,4,6,8}$ and the five slow intervals $I_{s}^{1,3,5,7,9}$

$$
\begin{aligned}
I_{f}^{2,4}: & =\left(-\xi_{*}^{1,2}-\frac{1}{\sqrt{\varepsilon}},-\xi_{*}^{1,2}+\frac{1}{\sqrt{\varepsilon}}\right), \quad I_{f}^{6,8}:=\left(\xi_{*}^{2,1}-\frac{1}{\sqrt{\varepsilon}}, \xi_{*}^{2,1}+\frac{1}{\sqrt{\varepsilon}}\right), \\
I_{s}^{1}: & =\left(-\infty,-\xi_{*}^{1}-\frac{1}{\sqrt{\varepsilon}}\right], \quad I_{s}^{3,7}:=\left[\mp \xi_{*}^{1,2}+\frac{1}{\sqrt{\varepsilon}}, \mp \xi_{*}^{2,1}-\frac{1}{\sqrt{\varepsilon}}\right], \\
I_{s}^{5}: & =\left[-\xi_{*}^{2}+\frac{1}{\sqrt{\varepsilon}}, \xi_{*}^{2}-\frac{1}{\sqrt{\varepsilon}}\right], \quad I_{s}^{9}:=\left[\xi_{*}^{1}+\frac{1}{\sqrt{\varepsilon}}, \infty\right) .
\end{aligned}
$$

The nine different regions are then

1. The dynamics take place exponentially close to the slow manifold $\mathcal{M}_{\varepsilon}^{-}: \xi \in I_{s}^{1}$.

2. The dynamics take place in the fast field: $\xi \in I_{f}^{2}$.

3. The dynamics take place exponentially close to $\mathcal{M}_{\varepsilon}^{+}: \xi \in I_{s}^{3}$.

4. The dynamics take place in the fast field: $\xi \in I_{f}^{4}$.

5. The dynamics take place exponentially close to $\mathcal{M}_{\varepsilon}^{-}: \xi \in I_{s}^{5}$.

6. The dynamics take place in the fast field: $\xi \in I_{f}^{6}$.

7. The dynamics take place exponentially close to $\mathcal{M}_{\varepsilon}^{+}: \xi \in I_{s}^{7}$.

8. The dynamics take place in the fast field: $\xi \in I_{f}^{8}$.

9. The dynamics take place exponentially close to $\mathcal{M}_{\varepsilon}^{-}: \xi \in I_{s}^{9}$.

The analysis of the formal construction is now nearly the same as for the standing one-pulse case (Sect. 2.2); the only difference is that it involves a bit more bookkeeping. However, qualitatively, nothing changes; for example we still have $\Delta_{f}^{2,4,6,8}(v, w, q, r)=\mathcal{O}(\sqrt{\varepsilon})$, the equivalent of (2.15). The homoclinic $v, w$-component on the slow manifolds are still governed by (2.10) and (2.11). Together with the usual boundary conditions, of which there are in total forty, we get 


$$
v_{2 p}(\xi)= \begin{cases}2 e^{\varepsilon \xi}\left(\sinh \left(\varepsilon \xi_{*}^{1}\right)-\sinh \left(\varepsilon \xi_{*}^{2}\right)\right)-1 & \text { in } 1 \\ -e^{-\varepsilon\left(\xi+\xi_{*}^{1}\right)}-e^{\varepsilon\left(\xi-\xi_{*}^{1}\right)}-2 e^{\varepsilon \xi}\left(\sinh \left(\varepsilon \xi_{*}^{2}\right)\right)+1 & \text { in } 3 \\ -e^{-\varepsilon\left(\xi+\xi_{*}^{1}\right)}+e^{-\varepsilon\left(\xi+\xi_{*}^{2}\right)}+e^{\varepsilon\left(\xi-\xi_{*}^{2}\right)}-e^{\varepsilon\left(\xi-\xi_{*}^{1}\right)}-1 & \text { in } 5 \\ -e^{-\varepsilon\left(\xi+\xi_{*}^{1}\right)}-e^{\varepsilon\left(\xi-\xi_{*}^{1}\right)}-2 e^{-\varepsilon \xi}\left(\sinh \left(\varepsilon \xi_{*}^{2}\right)\right)+1 & \text { in 7 } \\ 2 e^{-\varepsilon \xi}\left(\sinh \left(\varepsilon \xi_{*}^{1}\right)-\sinh \left(\varepsilon \xi_{*}^{2}\right)\right)-1 & \text { in } 9,\end{cases}
$$

and likewise

$$
w_{2 p}(\xi)= \begin{cases}2 e^{\frac{\varepsilon}{D} \xi}\left(\sinh \left(\frac{\varepsilon}{D} \xi_{*}^{1}\right)-\sinh \left(\frac{\varepsilon}{D} \xi_{*}^{2}\right)\right)-1 & \text { in } 1 \\ -e^{-\frac{\varepsilon}{D}\left(\xi+\xi_{*}^{1}\right)}-e^{\frac{\varepsilon}{D}\left(\xi-\xi_{*}^{1}\right)}-2 e^{\frac{\varepsilon}{D} \xi}\left(\sinh \left(\frac{\varepsilon}{D} \xi_{*}^{2}\right)\right)+1 & \text { in } 3 \\ -e^{-\frac{\varepsilon}{D}\left(\xi+\xi_{*}^{1}\right)}+e^{-\frac{\varepsilon}{D}\left(\xi+\xi_{*}^{2}\right)}+e^{\frac{\varepsilon}{D}\left(\xi-\xi_{*}^{2}\right)}-e^{\frac{\varepsilon}{D}\left(\xi-\xi_{*}^{1}\right)}-1 & \text { in } 5 \\ -e^{-\frac{\varepsilon}{D}\left(\xi+\xi_{*}^{1}\right)}-e^{\frac{\varepsilon}{D}\left(\xi-\xi_{*}^{1}\right)}-2 e^{-\frac{\varepsilon}{D} \xi}\left(\sinh \left(\frac{\varepsilon}{D} \xi_{*}^{2}\right)\right)+1 & \text { in } 7 \\ 2 e^{-\frac{\varepsilon}{D} \xi}\left(\sinh \left(\frac{\varepsilon}{D} \xi_{*}^{1}\right)-\sinh \left(\frac{\varepsilon}{D} \xi_{*}^{2}\right)\right)-1 & \text { in } 9 .\end{cases}
$$

By the reversibility symmetry (2.2), there are two Melnikov conditions (instead of the expected four), which are analogous to (2.17),

$$
\alpha v_{*}^{1,2}+\beta w_{*}^{1,2}+\gamma=0,
$$

with $v_{*}^{1,2}$ and $w_{*}^{1,2}$ defined in (5.1). When we define $A_{1}:=e^{-\varepsilon \xi_{*}^{1}}$ and $A_{2}:=e^{-\varepsilon \xi_{*}^{2}}$ $\left(0<A_{1}<A_{2}<1\right)$, and combine this with the above results (5.2), (5.3), and (5.4), we obtain

$$
\left\{\begin{array}{l}
-\alpha A_{1}^{2}+\alpha A_{1} A_{2}-\alpha A_{1} A_{2}^{-1}-\beta A_{1}^{\frac{2}{D}}+\beta A_{1}^{\frac{1}{D}} A_{2}^{\frac{1}{D}}-\beta A_{1}^{\frac{1}{D}} A_{2}^{-\frac{1}{D}}+\gamma=0 \\
+\alpha A_{2}^{2}-\alpha A_{1} A_{2}-\alpha A_{1} A_{2}^{-1}+\beta A_{2}^{\frac{2}{D}}-\beta A_{1}^{\frac{1}{D}} A_{2}^{\frac{1}{D}}-\beta A_{1}^{\frac{1}{D}} A_{2}^{-\frac{1}{D}}+\gamma=0 .
\end{array}\right.
$$

By adding and subtracting, this system can be transformed into

$$
\left\{\begin{array}{l}
G_{1}\left(A_{1}, A_{2}\right):=\alpha\left(A_{1}-A_{2}\right)^{2}+\beta\left(A_{1}^{\frac{1}{D}}-A_{2}^{\frac{1}{D}}\right)^{2}=0 \\
G_{2}\left(A_{1}, A_{2}\right):=\alpha\left(A_{2}^{2}-A_{1}^{2}\right)-2 \alpha A_{1} A_{2}^{-1}+\beta\left(A_{2}^{\frac{2}{D}}-A_{1}^{\frac{2}{D}}\right) 2-\beta A_{1}^{\frac{1}{D}} A_{2}^{-\frac{1}{D}}=-2 \gamma .
\end{array}\right.
$$

The above formal analysis gives rise to the following theorem.

Theorem 5.1 Let $(\alpha, \beta, \gamma, D, \tau, \theta, \varepsilon)$ be such that (5.6) has $K$ solution pairs $\left(A_{1}, A_{2}\right)$ with $0<A_{1}<A_{2}<1$. Let $\varepsilon>0$ be small enough. If $K=0$, then there are no homoclinic orbits to $P_{\varepsilon}^{-}$in (2.1) that have a structure as sketched in Fig. 12. If $K>0$, there are $K$ homoclinic orbits $\gamma_{2 p, j}^{-}(\xi), j \in\{1, \ldots, K\}$, to $P_{\varepsilon}^{-}$in (2.1) (with structure as in Fig. 12). These correspond to symmetric standing two-pulse solutions of (1.6).

Given the form of Eq. 5.6, it is natural to solve $A_{1}$ and $\gamma$ as functions of $A_{2}$ and the system parameters $\alpha, \beta$ and $D$. In Fig. 13, both $A_{1}$ and $\gamma$ are plotted. Note also that $G_{1}\left(A_{1}, A_{2}\right)$ cannot vanish in (5.6) if $\operatorname{sgn}(\alpha)=\operatorname{sgn}(\beta)$. Thus, there only exist homoclinic 2-pulse solutions if $\operatorname{sgn}(\alpha) \neq \operatorname{sgn}(\beta)$ - see Sect. 6 .

Proof of Theorem 5.1 A symmetric standing two-pulse $\gamma_{2 p, j}^{-}(\xi)$ is reversible (2.2) and we can therefore argue along the same lines as in the proof of Theorem 2.1. In fact, the proof of this theorem goes in essence very similar to that of Theorem 2.1. Therefore, we will omit most details. By the first Melnikov condition in (5.4), there exists a one-parameter family of orbits $\gamma_{\text {het }}^{1,-}\left(\xi ; v_{*}^{1} ; w_{*}^{1}\left(v_{*}^{1}\right)\right) \in W^{u}\left(P_{\varepsilon}^{-}\right) \cap W^{s}\left(\mathcal{M}_{\varepsilon}^{+}\right)$. We define the tube $\mathcal{T}_{1, *}^{-} \subset W^{u}\left(P_{\varepsilon}^{-}\right)$as the collection of orbits in $W^{u}\left(P_{\varepsilon}^{-}\right)$that are exponentially close to $\gamma_{h e t}^{1,-}\left(\xi ; v_{*}^{1} ; w_{*}^{1}\left(v_{*}^{1}\right)\right)$ for $\xi<-\xi_{*}^{1}$. 


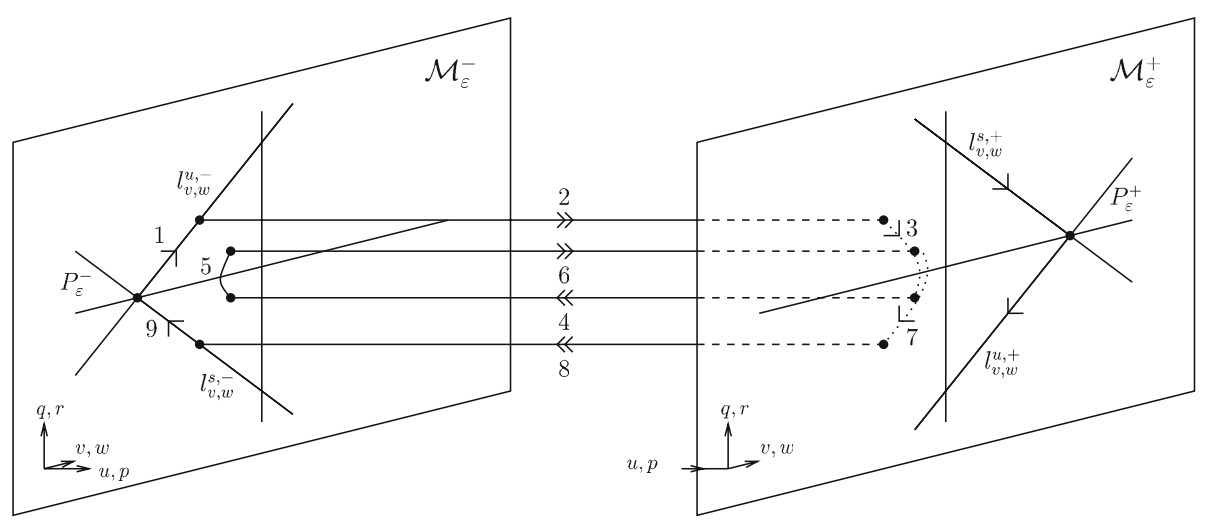

Fig. 12 A schematic sketch of a symmetric two-pulse $\gamma_{2 p, j}^{-}(\xi)$ homoclinic to $P_{\varepsilon}^{-}$
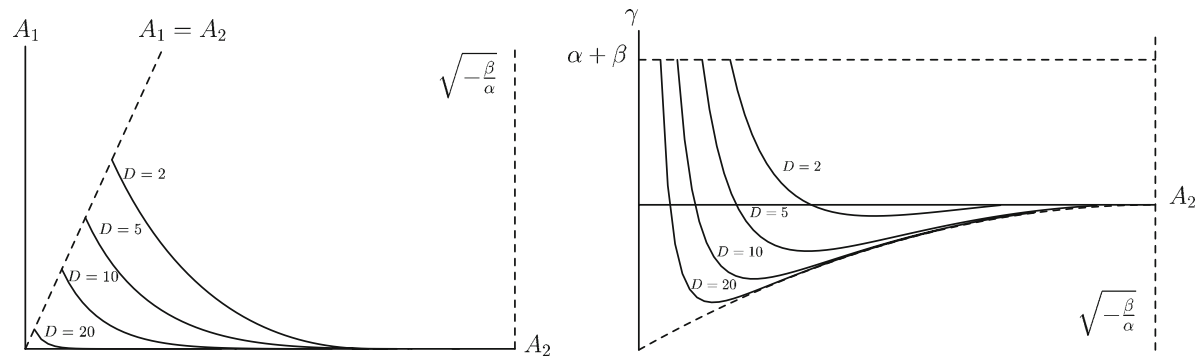

Fig. 13 In the left frame, $A_{1}$ is plotted as function of $A_{2}$ for several values of $D$. In the right frame, $\gamma$ is plotted as function of $A_{2}$ for the same values for $D$. The dashed curve represents the asymptotic behavior for $D$ large and is given by (5.12). The two-pulse orbits are typically created or annihilated in a saddle-node bifurcation—see Sect. 7, Fig. 15

All orbits in $\mathcal{T}_{1, *}^{-}$approach $\mathcal{M}_{\varepsilon}^{+}$and follow the slow flow on $\mathcal{M}_{\varepsilon}^{+}$for some 'time' (which may be infinite), after which they take off parallel (and exponentially close to) $W^{u}\left(\mathcal{M}_{\varepsilon}^{+}\right)$. In other words, near $\mathcal{M}_{\varepsilon}^{+} \mathcal{T}_{1, *}^{-}$is strongly stretched along the direction of $W^{u}\left(\mathcal{M}_{\varepsilon}^{+}\right)$. It thus follows by the application of the second Melnikov condition in (5.4) that $\mathcal{T}_{1, *}^{-}$intersects $W^{s}\left(\mathcal{M}_{\varepsilon}^{-}\right)$; the intersection $\mathcal{T}_{1, *}^{-} \cap W^{s}\left(\mathcal{M}_{\varepsilon}^{-}\right)$is again two-dimensional, i.e., it consists of a one-parameter family of orbits $\subset W^{u}\left(P_{\varepsilon}^{-}\right) \cap W^{s}\left(\mathcal{M}_{\varepsilon}^{-}\right)$. As in the proof of Theorem 2.1, it can now be shown that there is a unique orbit $\gamma_{0, *}^{2,-}(\xi) \subset \mathcal{T}_{1, *}^{-} \cap W^{s}\left(\mathcal{M}_{\varepsilon}^{-}\right)$that is homoclinic to $\mathcal{M}_{\varepsilon}^{-}$such that $\gamma_{0, *}^{2,-}(0) \in\{q=r=0\}$ - note that this also determines the position of the symmetry point $\xi=0$. Again, the algebra leading to the construction of $\gamma_{0, *}^{2,-}(\xi)$ is equivalent to the above analysis and yields at leading order (5.6). The existence of the 2-pulse homoclinic orbits $\gamma_{2 p, j}^{-}(\xi)$ now follows by arguments that are identical to those in Theorem 2.1. It is based on the construction of the sub-tube $\mathcal{T}_{2, *}^{-} \subset \mathcal{T}_{1, *}^{-}$around $\gamma_{0, *}^{2,-}(\xi)$, its symmetrical counterpart $\mathcal{T}_{2, *}^{+}$around the orbit $\gamma_{0, *}^{2,+}(\xi)$ and the application of the reversibility symmetry.

Remark 5.1 In the proof presented above we have used that the jump mid-points $v_{*}^{1,2}$ and $w_{*}^{1,2}$ satisfy certain constraints. In particular, $v_{*}^{1} \in(-1,0), w_{*}^{1}=-\frac{1}{\beta}\left(\alpha v_{*}^{1}+\gamma\right), v_{*}^{2} \in\left(v_{*}^{1}, V\right)$ and $w_{*}^{2}=-\frac{1}{\beta}\left(\alpha v_{*}^{2}+\gamma\right)$, where $V=-\frac{\xi_{*}^{1}+\xi_{*}^{2}}{2}-\frac{1}{2 \varepsilon} \log \left(1-e^{-2 \varepsilon \xi_{*}^{2}}+e^{-\varepsilon\left(\xi_{*}^{1}+\xi_{*}^{2}\right)}\right)$. These 
constraints arise naturally from the requirement that the tracked orbits lie on the correct side of the stable and unstable manifolds of the slow manifold, so that they can have a second pulse.

Remark 5.2 In our analysis we have focused on the existence of localized one- and twopulse patterns. As for instance in [4], the same geometrical approach as in the proofs of Theorems 2.1, 3.1 and 5.1 can be applied to establish the existence of many other kinds of stationary or travelling patterns, such as $N$-pulse solutions and various kinds of spatially periodic wave trains. We refrain from going into the details here. However, we do notice that these patterns can be stable and do play an important role in the dynamics of (1.7) - see section 7.1 and especially Fig. 15.

\subsection{The Existence of Two-Pulse Solutions}

Just as was the case for the $K$ of Theorem 2.1, it is, a priori, not clear whether there exist parameter combinations for which the $K$ of Theorem 5.1 is non-zero. To show that these parameter regimes do exist we first choose an explicit $D$ as an example, that is, we put $D=2$. Naturally, we also have to assume $\operatorname{sgn}(\alpha) \neq \operatorname{sgn}(\beta)$. With this special choice of $D$ we analyze (5.6). It transforms into

$$
\left\{\begin{array}{l}
H_{1}\left(A_{1}, A_{2}\right):=\alpha\left(A_{1}-A_{2}\right)^{2}+\beta\left(\sqrt{A_{1}}-\sqrt{A_{2}}\right)^{2}=0 \\
H_{2}\left(A_{1}, A_{2}\right):=\alpha\left(A_{2}^{2}-A_{1}^{2}\right)-2 \alpha \frac{A_{1}}{A_{2}}+\beta\left(A_{2}-A_{1}\right)-2 \beta \sqrt{\frac{A_{1}}{A_{2}}}=-2 \gamma .
\end{array}\right.
$$

Observe that the equality $H_{1}\left(A_{1}, A_{2}\right)=0$ does not depend on $\gamma$. Moreover, $\gamma$ only appears in the right hand side of $H_{2}\left(A_{1}, A_{2}\right)=-2 \gamma$. That is, $\gamma$ only shifts $H_{2}\left(A_{1}, A_{2}\right)$ up or down. So, instead of solving for $A_{1}$ and $A_{2}$ in terms of the unknown parameters $\alpha, \beta$ and $\gamma$, it is much easier to fix $\alpha, \beta$ and $A_{2}$ and to determine $A_{1}$ and $\gamma$ such that (5.7) is solved. Actually, by doing so, we impose, alongside $\alpha$ and $\beta$, one of the jump mid-points $\xi_{*}^{2}$ and try to locate the second jump mid-point $\xi_{*}^{1}$ and $\gamma$ such that (1.7) possesses a standing two-pulse. Of course, we could also choose to start with $\alpha, \beta$, and $A_{1}$ and determine $A_{2}$ and $\gamma$ that satisfy (5.7).

The zero of $H_{1}\left(A_{1}, A_{2}\right)$, for which $0<A_{1}<A_{2}$, is given by the relation

$$
\sqrt{A_{1}}+\sqrt{A_{2}}=\sqrt{-\beta / \alpha} .
$$

When we implement this into formula (5.7) for $H_{2}\left(A_{1}, A_{2}\right)$ we find, after some manipulation, a unique $\gamma$ :

$$
\gamma=\alpha-2 \alpha\left(1+A_{2}^{2}\right) \sqrt{-\frac{\beta}{\alpha A_{2}}}-\beta\left(1+3 A_{2}+\frac{1}{A_{2}}-\sqrt{-\frac{A_{2} \beta}{\alpha}}-\sqrt{-\frac{\beta}{\alpha A_{2}}}\right) .
$$

However, there are also restrictions on the choice of $A_{2}$. We need $0<A_{1}<A_{2}<1$. Therefore,

$$
-\frac{1}{4} \frac{\beta}{\alpha}<A_{2}<\min \left\{-\frac{\beta}{\alpha}, 1\right\} .
$$

We conclude that if $A_{2}$ satisfies (5.10), there is a $(\alpha, \beta, \gamma)$-parameter combination such that (5.7) is satisfied, i.e., such that a two-pulse solution exists. However, if (5.10) cannot be satisfied - which is the case when $|4 \alpha|<|\beta|$, there are no such two-pulse solutions.

This nonexistence result can be generalized to all $D>1$ :

Corollary 5.1 Let $\operatorname{sgn}(\alpha) \neq \operatorname{sgn}(\beta)$. There is an open region in $(\alpha, \beta, \gamma, D)$-space for which homoclinic two-pulse solutions as described in Theorem 5.1 exist. However, if $|\alpha| D^{2}<|\beta|$, then there are no such two-pulse solutions. 
Proof We start again by observing that $G_{1}\left(A_{1}, A_{2}\right)=0$ does not depend on $\gamma$, and that the $\gamma$ in $G_{2}\left(A_{1}, A_{2}\right)=-2 \gamma$ only shifts $G_{2}\left(A_{1}, A_{2}\right)$ up or down. So, again instead of solving $A_{1}$ and $A_{2}$ in terms of $\alpha, \beta$ and $\gamma$ via (5.6), we solve this equation for given $\alpha, \beta$ and $A_{2}$ with the unknown parameters $A_{1}$ and $\gamma$.

The condition $0<A_{1}<A_{2}<1$ yields the following generalization of (5.10)

$$
\left(-\frac{\beta}{\alpha D^{2}}\right)^{\frac{1}{2} \frac{D}{D-1}}<A_{2}<\min \left\{\left(-\frac{\beta}{\alpha}\right)^{\frac{1}{2} \frac{D}{D-1}}, 1\right\} .
$$

Here, the latter inequality ensures $A_{2} \in(0,1)$, and the former implies $A_{1}<A_{2}$. This interval is empty when $|\alpha| D^{2}<|\beta|$.

\subsection{Asymptotics for $D \rightarrow \infty$}

In this section, we analyze the large $D$ asymptotics of solutions of equation (5.6). From Fig. 13, we observe that, over a large portion of the interval $A_{2} \in(0,1)$, the solution curves for $A_{1}$ lie near the axis, and the solution curves for $\gamma$ lie near the lower dashed curve. Moreover, these curves approach their respective asymptotes as $D$ increases. We establish this result precisely in the following lemma:

Lemma 5.2 Assume that $\alpha>0>\gamma>\beta$. Then, for strictly $\mathcal{O}(1)$ values of $A_{2} \in$ $(0, \sqrt{-\beta / \alpha})$, as measured with respect to the asymptotically small parameter $1 / D$, the solutions $A_{1}=A_{1}\left(\alpha, \beta, A_{2}, D\right)$ and $\gamma=\gamma\left(\alpha, \beta, A_{2}, D\right)$ of Eq. 5.6 satisfy, to leading order,

$$
A_{1}=\left(1-\sqrt{-\frac{\alpha}{\beta}} A_{2}\right)^{D}, \quad \gamma=-\alpha\left(\sqrt{-\frac{\beta}{\alpha}}-A_{2}\right)^{2} \quad \text { as } \quad D \rightarrow \infty .
$$

The lower dashed curve in the right frame of Fig. 13 is this parabola of $\gamma$ as function of $A_{2}$. It is also useful to combine the results of (5.12) of this lemma into expressions for $A_{1}$ and $A_{2}$ in terms of the given system parameters. The result is, to leading order,

$$
A_{1}=\left(\frac{\gamma}{\beta}\right)^{\frac{D}{2}}, \quad A_{2}=\sqrt{-\frac{\beta}{\alpha}}-\sqrt{-\frac{\gamma}{\alpha}} .
$$

We also remark that in both frames there is a boundary layer at $A_{2}=A_{1}$, which is why we require $A_{2}$ to be strictly of $\mathcal{O}(1)$ for this result and we recall that the existence construction requires that $A_{1}<A_{2}$. In the boundary layer, the graph of $A_{1}$ limits on the diagonal, with a slope of -1 , while the graph of $\gamma$ is nearly vertical. Although the asymptotic analysis is not too involved, we refrain from going into the details here. Nevertheless, we notice that, by (5.6), $\gamma=\alpha+\beta$ in the limit $A_{2} \downarrow A_{1}$, see Fig. 13.

Proof of Lemma 5.2 We observe that, for $A_{2}$ strictly of $\mathcal{O}(1)$ in $(0,1)$, we may assume that

$$
A_{1}=C^{D}
$$

to leading order, for some $C \in(0,1)$. Indeed, if one instead assumed that $A_{1}=a \delta^{\sigma}$ to leading order, for $\delta=1 / D$ and for some $\sigma>0$, then from the first equation in (5.6) one would find that $A_{2}=0$ to leading order, which is a contradiction. Hence, with the assumption (5.13), the first equation in (5.6) becomes

$$
\alpha A_{2}^{2}+\beta(C-1)^{2}=0,
$$


to leading order, where we used that $A_{2}^{1 / D}=1+\mathcal{O}(1 / D)$ for $A_{2} \in(0,1)$, and that $(1 / D) \log \left(A_{2}\right) \ll C$. Solving, one finds, to leading order,

$$
A_{1}=\left(1-\sqrt{-\frac{\alpha}{\beta}} A_{2}\right)^{D},
$$

which is precisely the first formula of (5.12).

With the asymptotics for $A_{1}$ in hand, one may use the second formula in (5.6) to find the asymptotics for $\gamma$. To leading order,

$$
\gamma=-\frac{1}{2}\left[\alpha A_{2}^{2}+\beta\left(1-\left(1-\sqrt{-\frac{\alpha}{\beta}} A_{2}\right)^{2}\right)-2 \beta\left(1-\sqrt{-\frac{\alpha}{\beta}} A_{2}\right)\right] .
$$

Simplifying the right member, we find precisely the asymptotic result (5.12) for $\gamma$.

To conclude this section on the large $D$ asymptotics, we comment briefly on the form of the $W$ profile for stationary two-pulse solutions in the interval between the two pulses. From the above asymptotics, we find, to leading order,

$$
\varepsilon \xi=\mathcal{O}(1), \quad \varepsilon \xi_{*}^{2}=-\log A_{2}=\mathcal{O}(1), \quad \varepsilon \xi_{*}^{1}=-D \log \left(1-\sqrt{-\frac{\alpha}{\beta}} A_{2}\right)=\mathcal{O}(D) .
$$

Hence, from (5.3), we find in region 5, to leading order,

$$
\begin{aligned}
w_{2 p}(\xi) & =-e^{-\frac{\varepsilon}{D}\left(\xi+\xi_{*}^{1}\right)}+e^{-\frac{\varepsilon}{D}\left(\xi+\xi_{*}^{2}\right)}+e^{\frac{\varepsilon}{D}\left(\xi-\xi_{*}^{2}\right)}-e^{\frac{\varepsilon}{D}\left(\xi-\xi_{*}^{1}\right)}-1 \\
& =2 \sqrt{-\frac{\alpha}{\beta}} A_{2}-1 \\
& =1-2 \sqrt{\frac{\gamma}{\beta}} .
\end{aligned}
$$

Therefore, for each $A_{2} \in(0, \sqrt{-\beta / \alpha})$, the $W$-component is constant to leading order, where the constant is given by (5.16). Moreover, we observe that $W$ takes on all of the values in the interval $(-1,1)$, since the above analysis applies for all $A_{2} \in(0, \sqrt{-\beta / \alpha})$.

A stability analysis similar to that presented in [23] shows that the two-pulse solutions are stable for parameter combinations in the 'boundary layer'. However, they are unstable for parameter values near the dashed curve in the asymptotic regime studied in Lemma 5.2.

\section{The Two-Component Model}

In this section, we investigate the two-component $(U, V)$-subsystem of the three-component model, that is, we send $D$ to infinity and assume that the $W$-component is constant at $W=-1$ everywhere in the PDE (1.6). The PDE model reduces to

$$
\left\{\begin{array}{l}
U_{t}=\varepsilon^{2} U_{x x}+U-U^{3}-\varepsilon\left(\alpha_{2} V+\gamma_{2}\right) \\
\tau_{2} V_{t}=V_{x x}+U-V
\end{array}\right.
$$

with the same assumptions as before, $0<\varepsilon \ll 1,0<\tau_{2} \ll 1 / \varepsilon^{3}$ and $\alpha_{2}, \gamma_{2} \in \mathbb{R}$. Note that the notation for the parameters has the following correspondence with the parameters of the three-component model: $\alpha_{2}=\alpha, \tau_{2}=\tau$ and $\gamma_{2}=\gamma-\beta$. 
It can be shown with the same techniques used in this article that for $\tau_{2}=\mathcal{O}(1)$ the twocomponent system has standing one-pulse solutions homoclinic to $P_{2, \varepsilon}^{-}=\left(u_{2, \varepsilon}^{-}, 0, u_{2, \varepsilon}^{-}, 0\right)$ with $u_{2, \varepsilon}^{-}=-1+\frac{1}{2} \varepsilon\left(\alpha_{2}-\gamma_{2}\right)+\mathcal{O}\left(\varepsilon^{2}\right)$ if there exists an $A \in(0,1)$ satisfying

$$
\alpha_{2} A^{2}=\gamma_{2}+\mathcal{O}(\sqrt{\varepsilon})
$$

recall (2.22). Hence, we immediately observe that necessary conditions for a standing pulse homoclinic to $P_{2, \varepsilon}^{-}$to exist are that $\operatorname{sgn}\left(\alpha_{2}\right)=\operatorname{sgn}\left(\gamma_{2}\right)$ and $0<\left|\gamma_{2}\right|<\left|\alpha_{2}\right|$. Also, the existence of travelling pulse solutions to $P_{2, \varepsilon}^{-}$for large $\tau_{2}$ can be proved, and in the end it boils down to solving a system of equations which is a simplification of (3.13). Moreover, when we increase $\tau_{2}$ from an $\mathcal{O}(1)$-parameter to an $\mathcal{O}\left(\varepsilon^{-2}\right)$-parameter a travelling pulse solution bifurcates from a standing pulse solution at $\left(\tau_{2}\right)_{0, *}=\frac{1}{\varepsilon^{2}}\left(\hat{\tau}_{2}\right)_{0, *}=$ $\frac{1}{\varepsilon^{2}} \frac{2}{3} \sqrt{2}\left(\alpha_{2}-\gamma_{2}+\gamma_{2} \log \left(\frac{\gamma_{2}}{\alpha_{2}}\right)\right)$. This bifurcation can be supercritical, as well as subcritical. See also Sect. 4 and especially the proof of Lemma 4.2.

Finally, the two-component system possesses no symmetric standing two-pulse solutions to $P_{2, \varepsilon}^{-}$. Physically, this can be explained by the fact that the model has too few free constants (too few dimensions). The absence of two-pulse solutions is also plausible when we look at Theorem 5.1. There only exists a standing two-pulse solution if at least $\operatorname{sgn}(\alpha) \neq \operatorname{sgn}(\beta)$ and for the two-component system this condition cannot be fulfilled because there is no equivalent parameter for $\beta$ in the two-component system.

To summarize, we have shown that the two-component model also possesses stationary and travelling pulse solutions. However, it does not support two-pulse solutions.

Remark 6.1 There are two ways in which the three-component system (1.6) may limit on a two-component system, either by considering $W \rightarrow V$, associated to $D \downarrow 1$, or by $W \rightarrow W_{0}$, a constant when $D \rightarrow \infty$. In the former case one has to make the additional assumption that $\tau=\theta$. Since in most studies of systems like (1.1)/(1.6) $D \gg 1$ and $\tau \gg \theta$, we do not consider this limit here.

If one considers the limit $D \rightarrow \infty$ in Theorems 2.1 and 3.1 for one-pulse solutions, then it immediately follows that $W \rightarrow-1$ uniformly on $\mathbb{R}$ - see for instance (2.21). However, since the two-component limit cannot have standing two-pulse solutions, taking the limit $D \rightarrow \infty$ in Theorem 5.1 is less straightforward. In fact, this limit has already been discussed in section 5.3 (under the assumption that $A_{2}=\mathcal{O}(1)$ ). It follows from (5.15) that the width of the pulses in the two-pulse solution increases linearly with $D$, while the distance between the pulses approaches a finite limit. Thus, on bounded intervals, the two-pulse solution of the three-component system limits on a one-pulse solution of a two-component $(U, V)$-system that is homoclinic to $(U, V)=(+1,+1)$ (with $W \rightarrow 1-2 \sqrt{\gamma / \beta}$, the constant value given in (5.16)).

\section{Simulations, Conclusions and Discussion}

\subsection{Simulations}

In this section, we show the results of some numerical simulations to further illustrate the theory presented in this article and also to illustrate some of the basic pulse interactions and instabilities. These simulations are carried out using the numerical software presented in [1].

We already illustrated a stationary one-pulse solution in the left frame of Fig. 1. Therefore, we begin here with some travelling pulses of the type constructed in Sect. 3. The pulses shown in Fig. 14 exist for values of $\tau$ greater than the theoretically predicted value 

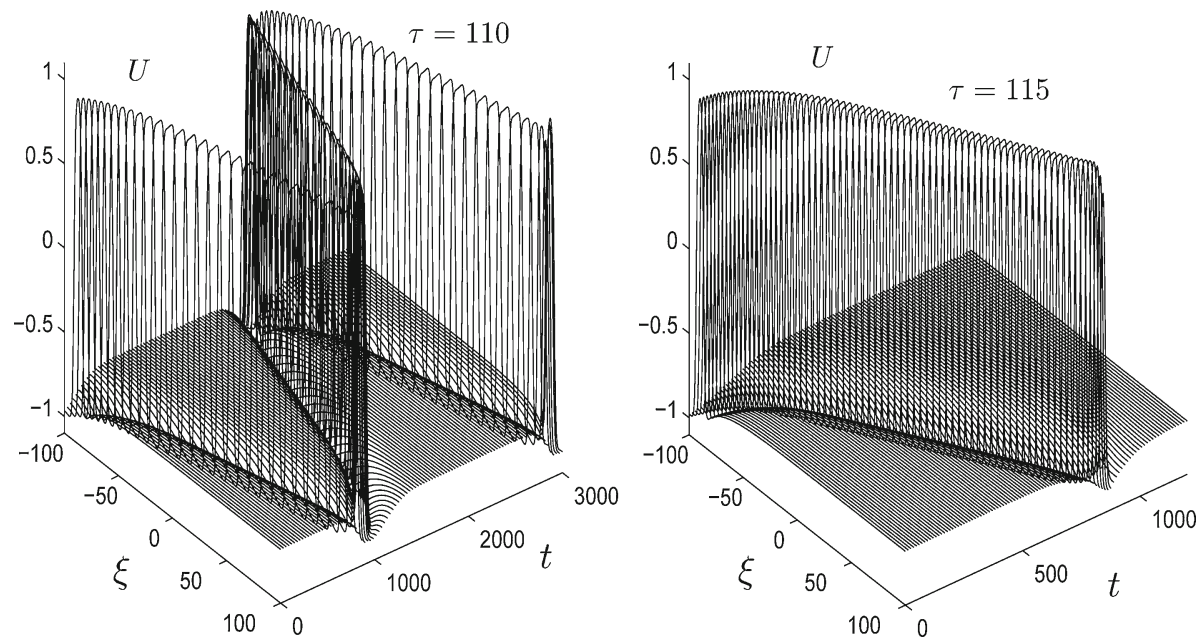

Fig. 14 Stable travelling pulses. The parameter values are $(\alpha, \beta, \gamma, D, \theta, \varepsilon)=(6,3,4,2,1,0.1)$, and $\tau$ is the bifurcation parameter. Here, we plotted a bouncing travelling pulse solution for $\tau=110$ and an annihilation of a travelling pulse for $\tau=115$

$\hat{\tau}_{*, 0}=0.59$ for the bifurcation in which travelling pulses are created (which translates into an unscaled $\tau_{*, 0}=59$ ). In the left frame, the travelling pulse collides with its mirror image pulse at the boundary, since the boundary conditions are of homogeneous Neumann type, and afterwards they repel each other. By contrast, in the right frame, the pulse and its mirror image collide and then annihilate. The changeover from repulsion to annihilation after the collision occurs at $\tau_{a n n}^{\text {num }}=112$. Finally, we observe that the numerically observed value of the bifurcation to travelling waves is $\tau_{*}^{\text {num }}=103$, which is within the relative error of magnitude $\mathcal{O}(1 / \varepsilon)=\mathcal{O}(10)$ of the leading order theoretical value $\tau_{*, 0}=59$. Of course, in these simulations $\varepsilon$ is not yet really small, and hence we checked that the value of $\tau_{*}^{\text {num }}$ decreases toward the value predicted by the leading order theory as $\varepsilon$ is decreased. For example, for $\varepsilon=0.01$, we find $\tau_{*}^{\text {num }}=5.95 \times 10^{3}$ (compared to $5.9 \times 10^{3}$ theoretically).

Next, we illustrate the theoretical results for stationary two-pulse solutions of (1.6), as derived in Sect. 5. For each of the four values of $\gamma=0.8,0.75,-0.25,-0.3$, Fig. 15 shows the corresponding stationary solution. Based on the simulations for these parameter values, we find that the homogeneous background state $U=-1$ undergoes a subcritical bifurcation into a two-pulse solution at $\gamma^{\text {num }}=0.78$. Likewise, due to the reversibility symmetry, the homogeneous state $U=+1$ bifurcates supercritically into a two-pulse solution at $\gamma^{\text {num }}=-0.78$, though we do not show this. In addition, we observe that, as we decrease $\gamma$ from 0.78 , the width of the pulses increases, until there is a bifurcation at $\gamma^{\text {num }}=-0.27$ at which the pulses coalesce, and the solution is $U=+1$ everywhere, except inside an interior layer and inside the layers at the boundaries of the computational interval. This solution is a spatially periodic solution. Moreover, the observed value for this coalescence of the pulses agrees well with the theoretically predicted value of $\gamma=-0.31$ for the saddle-node bifurcation, which occurs at the minimum in the curve shown in the right frame of Fig. 13.

One of the most commonly-encountered bifurcations that the pulse solutions undergo is a supercritical Hopf bifurcation in which the widths, and heights, of the pulses oscillate periodically in time. In Fig. 16, we show a breathing one-pulse in the left frame, and a breathing two-pulse in the right frame. For the one-pulse solution (with $\varepsilon=0.1$ ), the Hopf bifurcation occurs at $\tau_{H}^{\text {num }}=47$. Moreover, we find that the breather dies out for $\tau=49.8$. For 

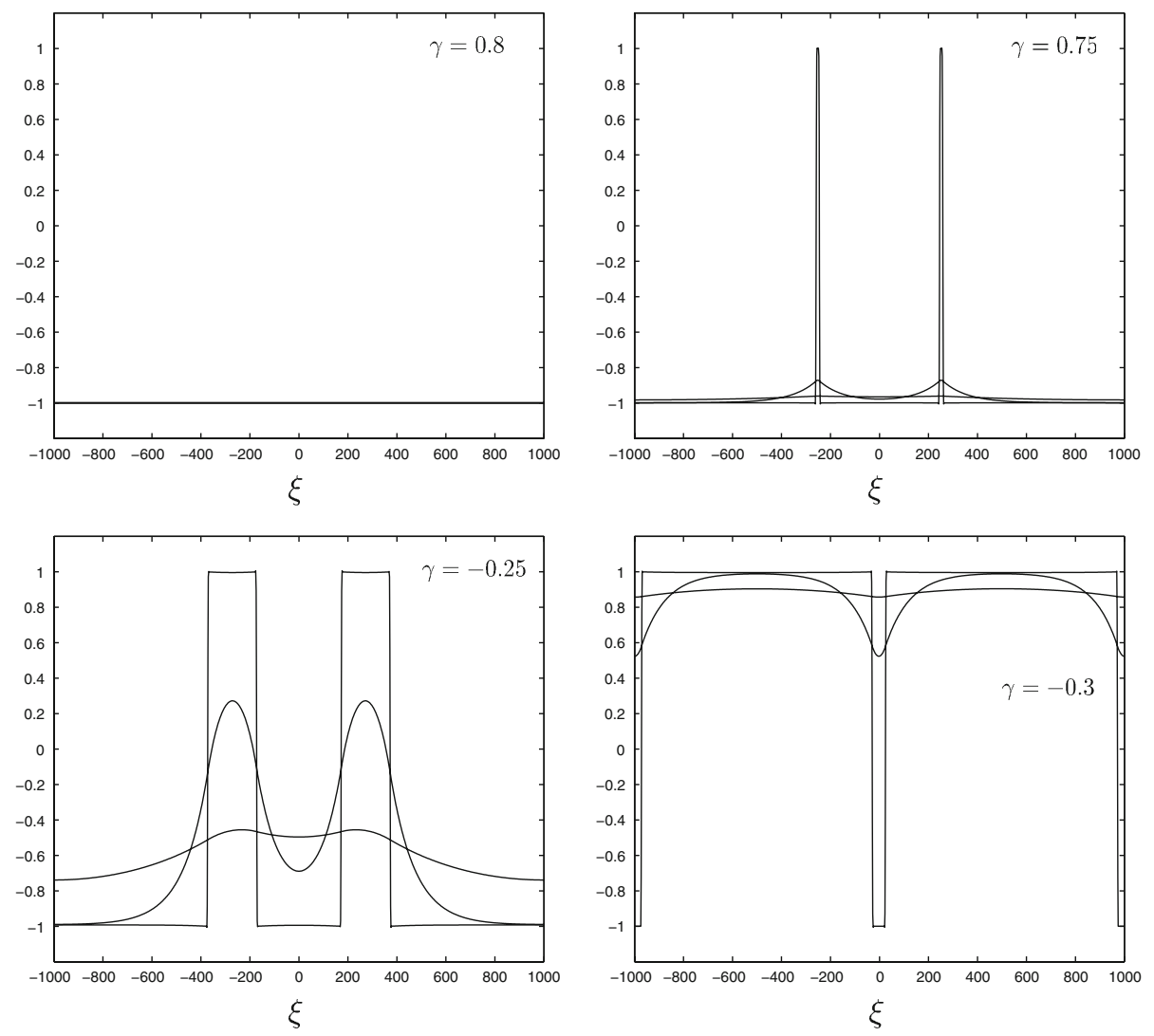

Fig. 15 Plots of the stationary solutions of the three-component model (1.6) for four values of $\gamma: \gamma=$ $0.8,0.75,-0.25,-0.3$. The values of the other parameters are $(\alpha, \beta, D, \tau, \theta, \varepsilon)=(2,-1,5,1,1,0.01)$

the two-pulse solution (with $\varepsilon=0.01$ ), the Hopf bifurcation takes place at $\tau_{H, 2 p}^{\text {num }}=4590$. Moreover, at $\tau=5060$, the breathing two-pulse solution becomes unstable and dies out. We note that we have observed breathing two-pulse solutions for which the pulse widths breath in an antisymmetric manner.

Scattering of pulses is also observed in the three-component model (1.7). In the left frame of Fig. 17, we show the $V$-component of a two-pulse solution in which the pulses initially approach each other, spend a substantial amount of time at a nearly constant distance from each other with a significantly-decreased amplitude, and then regain their original amplitudes and repel each other. The pulses continue to repel each other until they reflect off the boundary, and the process repeats. A similar phenomenon has been observed in $[15,16]$. There the unstable, stationary two-pulse, which the two-pulse data approaches, is called a 'scattor' (or 'separator'). The importance of a scattor stems from the observation made in $[15,16]$ that the forward evolution of two-pulse data that approaches it is determined by where that data lies with respect to the stable and unstable manifolds of the scattor or separator solution. The relation between scattors and the two-pulse solutions constructed in this article is the subject of future investigation.

We emphasize that the time interval shown in Fig. 17 is long and that the length of time where the two pulses are near to each other is also long in comparison to the time interval over which the pulses move an $\mathcal{O}(1)$ distance. Moreover, we found that the duration of this 

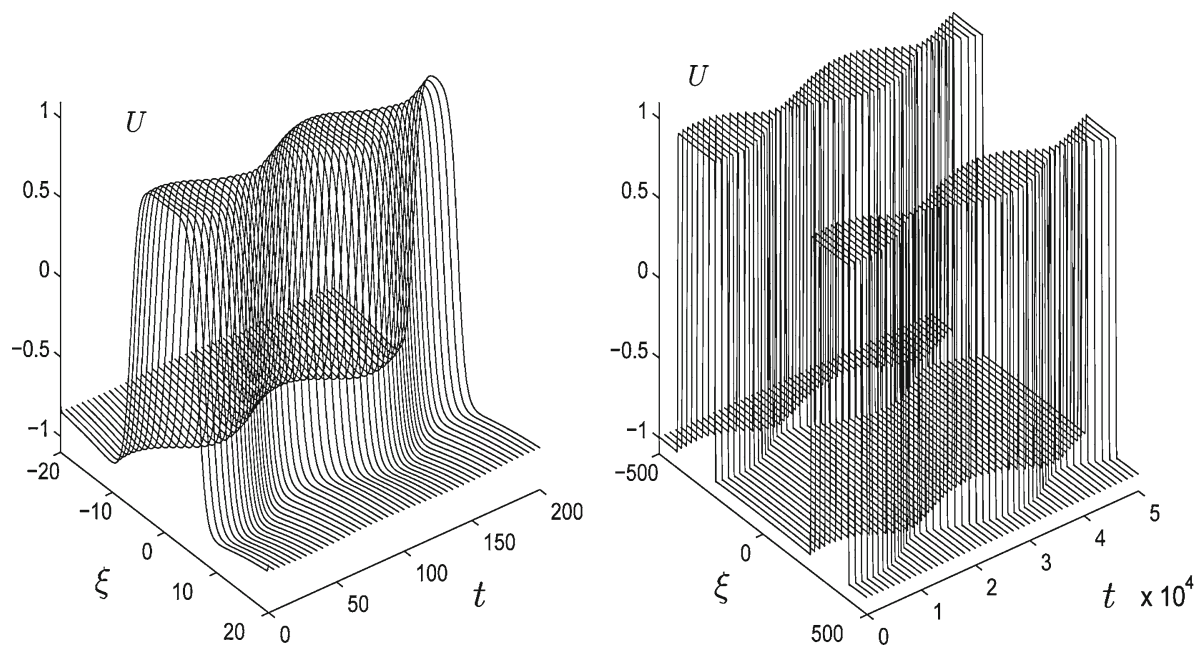

Fig. 16 Stable breathing one-pulse and two-pulse solutions. For the simulation shown in the left frame, $\tau=49.7$, and the other parameters are $(\alpha, \beta, \gamma, D, \theta, \varepsilon)=(6,3,4,10,1,0.1)$. Also, we note that the interval used in the simulation is $\xi \in[-100,100]$, however we have displayed only a subinterval to better display the breathing behavior. For the simulation shown in the right frame, $\tau=5000$, and the other parameters are $(\alpha, \beta, \gamma, D, \theta, \varepsilon)=(2.2,-1,0,10,1,0.01)$. Also, we note that the interval used in the simulation is $\xi \in[-1000,1000]$
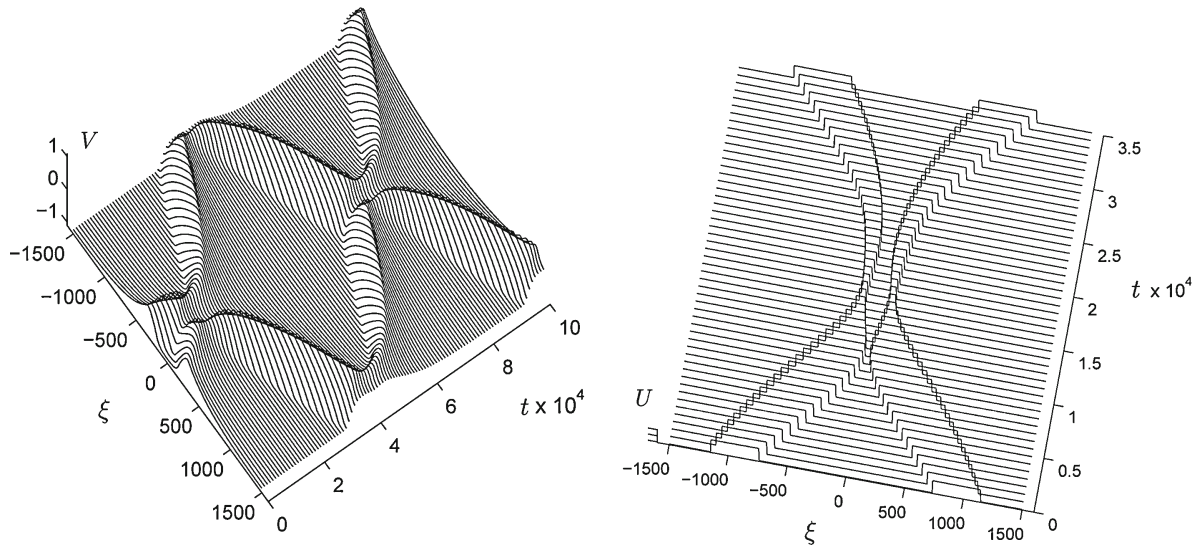

Fig. 17 Scattering of two pulses. In the left frame, we show the $V$-component over a long time interval, and in the right frame we show the $U$-component during the third central scattering event (not shown for the $V$-component). The parameter values are $(\alpha, \beta, \gamma, D, \tau, \theta, \varepsilon)=(6,3,2,2,6500,1,0.01)$

time interval can be changed by varying the parameter values. Finally, it is worth noting that, during the time that the two pulses are near the boundaries, they are also near their counterparts across the boundary, in what also appears to be a scattor state.

To conclude this brief section illustrating some of the pulse dynamics, we show the spatiotemporal evolution of four-pulse initial data in Fig. 18. Initially, the four pulses approach each other. Then, they start to breath in a time-periodic manner, until finally the middle two pulses die out and the two remaining pulses become stationary. In the right frame, we have zoomed in on the time interval containing the last few breathing periods, and here the destabilization 

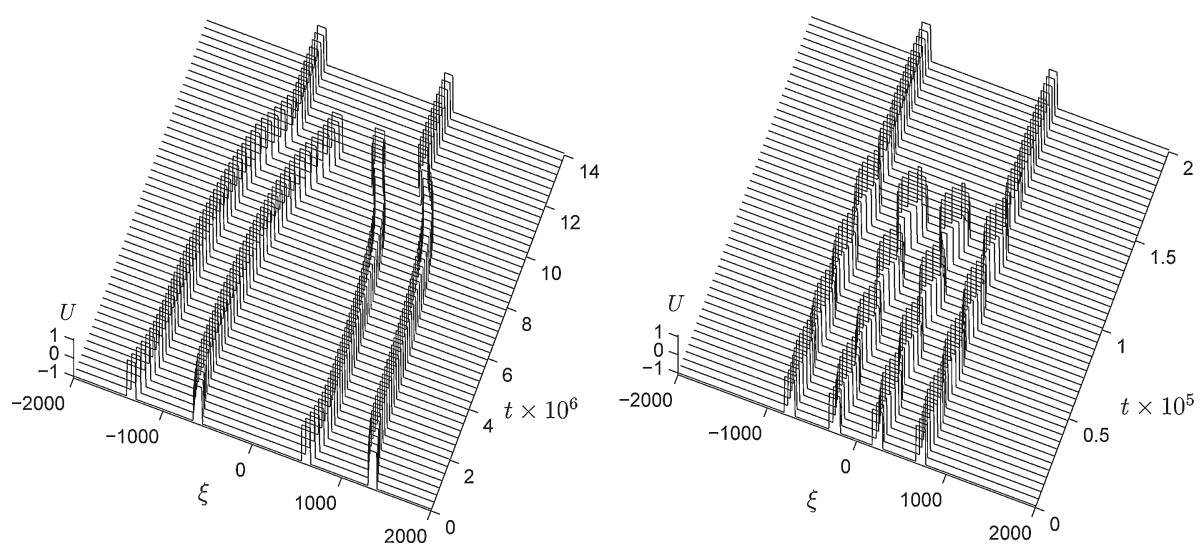

Fig. 18 The spatio-temporal dynamics of a solution with symmetric four-pulse initial data. The parameter values are $(\alpha, \beta, \gamma, D, \tau, \theta, \varepsilon)=(2.1,-1,0,5,3900,1,0.01)$. Note that we actually give an asymmetric two-pulse as initial condition and just 'mirrored' the domain, this can be done because of the Neumann boundary conditions. Note that the time interval shown in the left frame is so long that the breathing is not visible. Therefore, in the right frame, we zoomed in on the time interval $\left[11.2 \times 10^{6}, 11.4 \times 10^{6}\right]$ for the same solution, so that the breathing is clearly visible

process is visible in detail. The maximal widths per period of the inner two pulses increase as the time of annihilation gets closer and closer, while the minimal widths decrease. One can see that during the final oscillation the maximal pulse widths exceed the lengths of the gaps between the pulses. Finally, stepping back out to the time scale shown in the left frame, one sees that the time asymptotic state is a stable two-pulse solution of the type constructed in Sect. 3 , with pulse centers well inside $\xi=-1000$ and $\xi=1000$ on the domain $\xi \in[-2000,2000]$.

\subsection{Conclusions and Discussion}

In this article, we established the existence of stationary and travelling one-pulse solutions of the three-component model (1.6), as well as the existence of stationary two-pulse solutions. The main results are presented in Theorem 2.1, Lemma 2.1, and Theorem 3.1 for the one-pulse solutions, and in Theorem 5.1 for the two-pulse solutions. Moreover, we studied various bifurcations of these solutions, including the saddle-node bifurcation in which the stationary one-pulse solutions are created (see Theorem 2.1), the bifurcation from stationary to travelling one-pulses (showing that this may be either subcritical or supercritical depending on the system parameters, see Lemma 4.1 and Corollary 4.2), and the saddle-node bifurcation of two-pulse solutions, see Fig. 13.

In the course of this analysis, we also showed that this three-component system constitutes an ideal system on which to study pulse dynamics. On one hand, it is sufficiently simple for analysis using geometric singular perturbation theory, with all of the reaction terms, except for one, being linear. On the other hand, it is sufficiently nonlinear to support rich pulse dynamics. Indeed, the extent of this richness was first demonstrated in $[15,16,18,22]$, and these interacting pulse solutions exist also for the scaled Eq. 1.6 studied here. We think that the analysis presented in this work offers a useful starting point for the analysis of these various pulse interaction scenarios.

Finally, we considered the limit in which the three-component system (1.6) reduces to the more classical two-component system (6.1). This two-component system is almost the same as the FitzHugh-Nagumo equations, except that the second species (inhibitor) also diffuses 
here. It is shown that the two-component system possesses only the one-pulse solutions, and not the two-pulse solutions of the type studied here. Hence, the addition of the third component, as introduced in [22], is essential for the existence of two-pulse solutions.

Stability of the solutions studied here is an important topic, as is demonstrated for instance by the bifurcations to breathing pulses shown in Fig. 16. This is the topic of a companion paper [23], in which we use the Evans function and the NonLocal Eigenvalue Problem method [3] to carry out this analysis.

The methods and analysis of this article can be extended to carry out the analysis of pulse solutions in the three-component model with heterogeneity that is studied in [25]. There, heterogeneity is introduced in (1.1) by making the constant term in the $U$-component vary in space according to a smoothed out step function. The heterogeneity induces interesting new pulse dynamics, such as rebounding off defects, pinning by defects, and penetration of defects, as observed in numerical simulations. The invariant manifold theory from the field of geometric singular perturbation theory that we have used in this article, as well as the Melnikov conditions that we used, can also be applied to these types of heterogeneous systems, so that the pulse solutions may be constructed. In conjunction with these observations, we point to an earlier example in which geometric singular perturbation theory was used to establish the existence of standing wave solutions in a RD model of the Fabry-Perot interferometer, which involves spatially dependent coefficients. See [21].

Acknowledgements The authors thank Y. Nishiura for introducing us to the three-component model and for stimulating conversations. We thank P. Zegeling for valuable assistance with the software [1] used in the numerical simulations. A.D., P.v.H. and T.K. gratefully acknowledge support from the Netherlands Organization for Scientific Research (NWO). T. J. Kaper gratefully acknowledges support from the National Science Foundation through grant DMS-0606343, and thanks the CWI for its hospitality.

Open Access This article is distributed under the terms of the Creative Commons Attribution Noncommercial License which permits any noncommercial use, distribution, and reproduction in any medium, provided the original author(s) and source are credited.

\section{References}

1. Blom, J.G., Zegeling, P.A.: Algorithm 731: a moving-grid interface for systems of one-dimensional time-dependent partial differential equations. ACM Trans. Math. Softw. 20, 194-214 (1994)

2. Bode, M., Liehr, A.W., Schenk, C.P., Purwins, H.-G.: Interaction of dissipative solitons: particle-like behavior of localized structures in a three-component reaction-diffusion system. Physica D 161, 45-66 (2002)

3. Doelman, A., Gardner, R.A., Kaper, T.J.: Large stable pulse solutions in reaction-diffusion equations. Indian Univ. Math. J. 50(1), 443-507 (2001)

4. Doelman, A., Kaper, T.J., Ploeg, H.van der : Spatially periodic and aperiodic multi-pulse patterns in the one-dimensional Gierer-Meinhardt equation. Meth. Appl. Anal. 8(3), 387-414 (2001)

5. Doelman, A., Gardner, R.A., Kaper, T.J.: A stability index analysis of 1-D patterns of the Gray-Scott model. Mem. AMS 155(737) (2002)

6. Doelman, A., Iron, D., Nishiura, Y.: Destabilization of fronts in a class of bi-stable systems. SIAM Math. J. Anal. 35(6), 1420-1450 (2004)

7. Evans, J.W., Fenichel, N., Feroe, J.A.: Double impulse solutions in nerve axon equations. SIAM J. Appl. Math. 42, 219-234 (1982)

8. Fenichel, N.: Persistence and smoothness of invariant manifolds for flows. Indiana Univ. Math. J. 21, 193-226 (1971)

9. Fenichel, N.: Geometric singular perturbation theory for ordinary differential equations. J. Differential Equations 31, 53-98 (1979)

10. Hastings, S.: Single and multiple pulse waves for the FitzHugh-Nagumo equations. SIAM J. Appl. Math. 42, 247-260 (1982) 
11. Jones, C.K.R.T.: Geometric singular perturbation theory. In: Johnson, R. (ed.) Dynamical Systems, Montecatini Terme, 1994: Lecture Notes in Mathematics, vol. 1609. Springer-Verlag (1995)

12. Jones, C.K.R.T., Kopell, N.: Tracking invariant manifolds with differential forms in singularly perturbed systems. J. Differential Equations 108(1), 64-88 (1994)

13. Jones, C.K.R.T., Kaper, T.J., Kopell, N.: Tracking invariant manifolds up to exponentially small errors. SIAM J. Math. Anal. 27(2), 558-577 (1996)

14. Kaper, T.J.: An introduction to geometric methods and dynamical systems theory for singular perturbation problems. Proc. Sympos. Appl. Math. 56, 85-131 (1999)

15. Nishiura, Y., Teramoto, T., Ueda, K.-I.: Scattering and separators in dissipative systems. Phys. Rev. E 67, 056210 (2003)

16. Nishiura, Y., Teramoto, T., Ueda, K.-I.: Scattering of traveling spots in dissipative systems. CHAOS 15(4), 047509 (2005)

17. Nishiura, Y., Teramoto, T., Yuan, X., Ueda, K.-I.: Dynamics of traveling pulses in heterogeneous media. CHAOS 17(3), 031704 (2007)

18. Or-Guil, M., Bode, M., Schenk, C.P., Purwins, H.-G.: Spot bifurcations in three-component reactiondiffusion systems: the onset of propagation. Phys. Rev. E 57, 6432-6437 (1998)

19. Rasker, A.P.: Pulses in a bi-stable reaction-diffusion system. MA Thesis, KdV Inst., Univ. Amsterdam, The Netherlands (2005)

20. Robinson, C.: Sustained resonance for a nonlinear system with slowly-varying coefficients. SIAM J. Math. Anal. 14, 847-860 (1983)

21. Rubin, J., Jones, C.K.R.T.: Existence of standing pulse solutions to an inhomogeneous reaction-diffusion system. J. Dynam. Differential Equations 10, 1-35 (1998)

22. Schenk, C.P., Or-Guil, M., Bode, M., Purwins, H.-G.: Interacting pulses in three-component reactiondiffusion systems on two-dimensional domains. PRL 78(19), 3781-3784 (1997)

23. van Heijster, P., Doelman, A., Kaper, T.J.: Pulse dynamics in a three-component system: stability and bifurcations. To appear in Physica D (2008)

24. Yang, L., Zhabotinsky, A.M., Epstein, I.R.: Jumping solitary waves in an autonomous reaction-diffusion system with subcritical wave instability. Phys. Chem. Chem. Phys. 8, 4647-4651 (2006)

25. Yuan, X., Teramoto, T., Nishiura, Y.: Heterogeneity-induced defect bifurcation and pulse dynamics for a three-component reaction-diffusion system. Phys. Rev. E 75, 036220 (2007) 